Historic, Archive Document

Do not assume content reflects current scientific knowledge, policies, or practices. 

a SDil

A42

Recur

United States

Department of

Agriculture

Forest Service

Rocky Mountain

Forest and Range

Experiment Station

Fort Collins,

Colorado 80526

General Technical

Report RM-162

U4:5
Forest Vegetation on National Forests in the Rocky Mountain and Intermountain Regions: Habitat Types and Community Types

\author{
Robert R. Alexander
}

霓磷

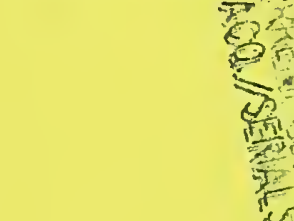

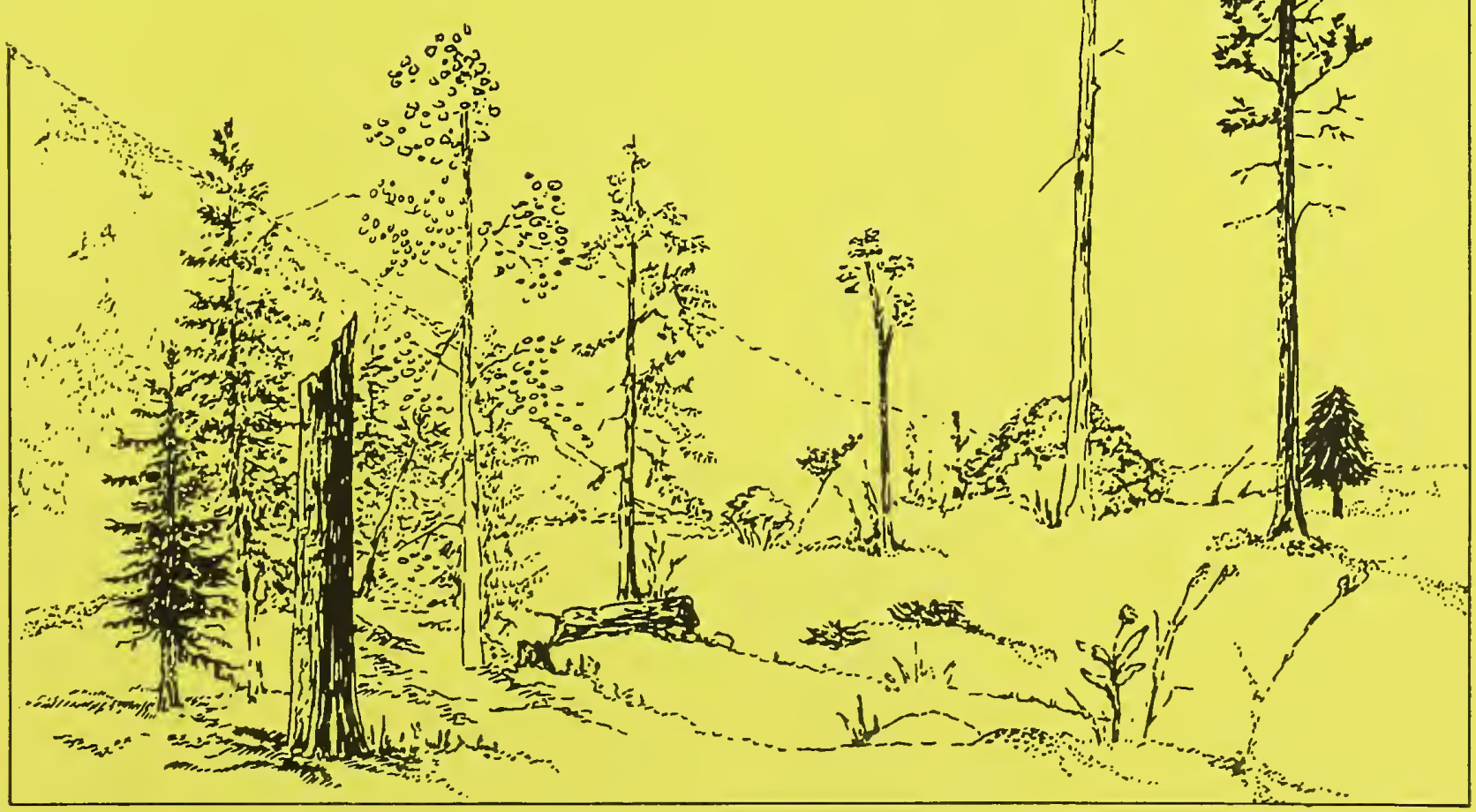




\title{
Forest Vegetation on National Forests in the Rocky Mountain and Intermountain Regions: Habitat Types and Community Types
}

\author{
Robert R. Alexander, Chief Silviculturist \\ Rocky Mountain Forest and Range Experiment Station ${ }^{1}$
}

\begin{abstract}
Habitat types and community types and their phases for the major forest tree species in the Rocky Mountain and Intermountain regions are tabulated. Included are the name(s), general location, elevation, relative site, successional status, principal tree and undergrowth associates, and the authority.
\end{abstract}




\title{
Forest Vegetation on National Forests in the Rocky Mountain and Intermountain Regions: Habitat Types and Community Types
}

\author{
Robert R. Alexander
}

In 1985, a list was published that documented habitat types, community types, and plant communities in the Rocky Mountain and Intermountain regions in which interior Pinus ponderosa, interior Pseudotsuga menziesii, interior Abies concolor, Picea pungens, Populus tremuloides, Pinus contorta, Picea engelmannii, and Abies lasiocarpa occurred as either a major climax, co-climax, minor climax, or major seral species (Alexander 1985). This paper is intended to supplement the 1985 publication by including newly available data and data on phases omitted in the 1985 publication. Moreover, the habitat and community types in the series in which the naming species occurs, listed in the 1985 publication, are repeated for the readers convenience. In addition to the species listed above, forested habitat types and community types and their phases are included that are dominated by Pinus leiophylla, Pinus engelmannii, Pinus strobiformis, Abies grandis, Thuja plicata, Tsuga heterophylla, Picea glauca, Pinus flexilis, Pinus aristata, Tsuga mertensiana, Pinus albicaulis, and Larix lyalli. Woodland and riparian habitat types and community types are not included, because these classifications are incomplete.

Table A1 lists the identified habitat types and community types and their phases for all forest tree species in the Rocky Mountain and Intermountain regions. Also included are the general location, elevation, site, successional status, principal tree and undergrowth associates, and the authority for the classification.

Some of the terms used in the table are clarified as follows.

1. Habitat type is the basic unit in classifying lands based on potential (climax) natural vegetation. A "habitat type" represents, collectively, all parts of the landscape that support, or have the potential of supporting, the same climax vegetation. The climax vegetation upon which the classification is based is called a "plant association." The first level of the classification is the "series," which is the grouping of all plant associations having the same overstory (climax) dominants. Fоr example, all habitat types with Pinus leiophylla as the potential climax dominant are grouped into the Pinus leiophylla series.

2. Habitat types within a series are distinguished on the basis of undergrowth unions, the smallest "structural unit" of the vegetation. Each union comprises one or more undergrowth species that exhibit similar microenvironmental requirements.

3. The term "community type" has been used to identify vegetation that may be either (1) climax, but about which there is uncertainty; (2) seral, but the trend toward climax is not evident; or (3) the recognized plant com- munity in place, which varies at any given time. Community types have one or more overstory dominants and characteristic undergrowth species. The undergrowth may be climax, but the overstory dominants often are long-lived, seral species that may be self-perpetuating because of repeated disturbance that prevents or slows down the succession to climax vegetation.

4. The description of the site (e.g., warm dry, cool dry) refers only to the series and location and, therefore, is relative. Obviously, a warm dry Pinus ponderosa site is not the same as a warm dry Abies lasiocarpa site.

5. In those habitat types where more than one phase is recognized, the typic phase is listed first, followed by the other phases. Phase is a subdivision of a habitat type representing a characteristic variation in climax vegetation and environmental conditions.

6. Synonyms of habitat types and closely related habitat types (which may be the same habitat type) are included within brackets.

7. Under the heading "Principal undergrowth species," the undergrowth species for which the habitat type is named is listed first, followed in order by shrubs, graminoids, and forbs.

\section{References}

Alexander, Billy G., Jr.; Fitzhugh, E. Lee; Ronco, Frank, Jr.; Ludwig, John A. 1987. A classification of forest habitats of the northern portion of the Cibola National Forest, New Mexico. Gen. Tech. Rep. RM-143. Fort Collins, CO: U.S. Department of Agriculture, Forest Service, Rocky Mountain Forest and Range Experiment Station. 35 p.

Alexander, Billy G., Jr.; Ronco, Frank, Jr.; Fitzhugh, E. Lee; Ludwig, John A. 1984a. A classification of forest habitat types on the Lincoln National Forest, New Mexico. Gen. Tech. Rep. RM-104. Fort Collins, CO: U.S. Department of Agriculture, Forest Service, Rocky Mountain Forest and Range Experiment Station. 29 p.

Alexander, Billy G., Jr.; Ronco, Frank, Jr.; White, Alan S.; Ludwig, John A. 1984b. Douglas-fir habitat types of northern Arizona. Gen. Tech. Rep. RM-108. Fort Collins, CO: U.S. Department of Agriculture, Forest Service, Rocky Mountain Forest and Range Experiment Station. 13 p.

Alexander, Robert R. 1985. Major habitat types, community types, and plant communities in the Rocky Mountains. Gen. Tech. Rep. RM-123. Fort Collins, CO: U.S. Department of Agriculture, Forest Service, Rocky Mountain Forest and Range Experiment Station. $105 \mathrm{p}$. 
Alexander, Robert R.; Hoffman, George R.; Wirsing, John M. 1986. Forest vegetation of the Medicine Bow National Forest in southeastern Wyoming: a habitat type classification. Res. Pap. RM-271. Fort Collins, CO: U.S. Department of Agriculture, Forest Service, Rocky Mountain Forest and Range Experiment Station. 39 p.

Cooper, Steven V.; Neiman, Kenneth E.; Steele, Robert; Roberts, David W. 1987. Forest habitat types of northern Idaho: a second approximation. Gen. Tech. Rep. INT-236. Ogden, UT: U.S. Department of Agriculture, Forest Service, Intermountain Research Station. 135 p.

Daubenmire, R.; Daubenmire Jean B. 1968. Forest vegetation of eastern Washington and northern Idaho. Tech. Bull. 60. Pullman, WA: Washington Agricultural Experiment Station, Washington State University. 104 p.

DeVelice, Robert L.; Ludwig, John A. 1983. Forest habitat types south of the Mogollon Rim, Arizona and New Mexico. Final Report. CA K-28-240. Fort Collins, CO: U.S. Department of Agriculture, Forest Service, Rocky Mountain Forest and Range Experiment Station. 48 p. plus appendixes.

DeVelice, Robert L.; Ludwig, John A.; Moir, William H.; Ronco, Frank, Jr. 1986. A classification of forest habitat types of northern New Mexico and southern Colorado. Gen. Tech. Rep. RM-131. Fort Collins, CO: U.S. Department of Agriculture, Forest Service, Rocky Mountain Forest and Range Experiment Station. 59 p.

Fitzhugh, E. Lee; Moir, William H.; Ludwig, John A.; Ronco, Frank, Jr. 1987. Forest habitat types in the Apache, Gila, and part of the Cibola National Forests in Arizona and New Mexico. Gen. Tech. Rep. RM-145. Fort Collins, CO: U.S. Department of Agriculture, Forest Service, Rocky Mountain Forest and Range Experiment Station. $116 \mathrm{p}$.

Hanks, Jess P.; Fitzhugh, E. Lee; Hanks, Sharon R. 1983. A habitat type classification system for ponderosa pine forests of northern Arizona. Gen. Tech. Rep. RM-97. Fort Collins, CO: U.S. Department of Agriculture, Forest Service, Rocky Mountain Forest and Range Experiment Station. 22 p.

Hansen, Paul L.; Hoffman, George R. 1988. The vegetation of the Grand River/Cedar River, Sioux, and Ashland Districts of the Custer National Forest: a habitat type classification. Gen. Tech. Rep. RM-157. Fort Collins, CO: U.S. Department of Agriculture, Forest Service, Rocky Mountain Forest and Range Experiment Station. $68 \mathrm{p}$.

Hansen, Paul L.; Hoffman, George R.; Bjugstad, Ardell J. 1984. The vegetation of Theodore Roosevelt National Park, North Dakota: a habitat type classification. Gen. Tech. Rep. RM-113. Fort Collins, CO: U.S. Department of Agriculture, Forest Service, Rocky Mountain Forest and Range Experiment Station. 35 p.

Hess, Karl; Alexander, Robert R. 1986. Forest vegetation of the Arapaho and Roosevelt National Forests in north-central Colorado: a habitat type classification. Res. Pap. RM-266. Fort Collins, CO: U.S. Department of Agriculture, Forest Service, Rocky Mountain Forest and Range Experiment Station. 48 p.

Hess, Karl; Wasser, Clinton H. 1982. Grassland, shrubland and forestland habitat types of the White River-
Arapaho National Forest. Final Report. 53-82 FT-1-19. Fort Collins, CO: U.S. Department of Agriculture, Forest Service, Rocky Mountain Forest and Range Experiment Station. $335 \mathrm{p}$.

Hoffman, George R. 1988. Forest vegetation of the Grand Mesa and Uncompahgre National Forests in western Colorado: a habitat type classification. Personal correspondance on file. Fort Collins, CO: U.S. Department of Agriculture, Forest Service, Rocky Mountain Forest and Range Experiment Station.

Hoffman, George R.; Alexander, Robert R. 1976. Forest vegetation of the Bighorn Mountains, Wyoming: a habitat type classification. Res. Pap. RM-170. Fort Collins, CO: U.S. Department of Agriculture, Forest Service, Rocky Mountain Forest and Range Experiment Station. 38 p.

Hoffman, George R.; Alexander, Robert R. 1980. Forest vegetation of the Routt National Forest in northwestern Colorado: a habitat type classification. Res. Pap. RM-221. Fort Collins, CO: U.S. Department of Agriculture, Forest Service, Rocky Mountain Forest and Range Experiment Station. 41 p.

Hoffman, George R.; Alexander, Robert R. 1983. Forest vegetation of the White River National Forest in western Colorado: a habitat type classification. Res. Pap. RM-249. Fort Collins, CO: U.S. Department of Agriculture, Forest Service, Rocky Mountain Forest and Range Experiment Station. 36 p.

Hoffman, George R.; Alexander, Robert R. 1987. Forest vegetation of the Black Hills National Forest of South Dakota and Wyoming. Res. Pap. RM-276. Fort Collins, CO: U.S. Department of Agriculture, Forest Service, Rocky Mountain Forest and Range Experiment Station. 48 p.

Johnston, Barry C.; Hendzel, Leonard. 1985. Examples of aspen treatment, succession and management in western Colorado. Report. Denver CO: U.S. Department of Agriculture, Forest Service, Rocky Mountain Region. 164 p.

Komarkova, Vera; Alexander, Robert R.; Johnston, Barry C. 1988. Forest vegetation of the Gunnison and parts of the Uncompahgre National Forests: a preliminary habitat type classification. Gen. Tech. Rep. Fort Collins, CO: U.S. Department of Agriculture, Forest Service, Rocky Mountain Forest and Range Experiment Station. (Manuscript in preparation).

Mauk, Ronald L.; Henderson, Jan A. 1984. Coniferous forest habitat types of northern Utah. Gen. Tech. Rep. INT-170. Ogden, UT: U.S. Department of Agriculture, Forest Service, Intermountain Forest and Range Experiment Station. $89 \mathrm{p}$.

Moir, William H.; Ludwig, John A. 1979. A classification of spruce-fir and mixed conifer habitat types of Arizona and New Mexico. Res. Pap. RM-207. Fort Collins, CO: U.S. Department of Agriculture, Forest Service, Rocky Mountain Forest and Range Experiment Station. 47 p.

Mueggler, Walter F. 1987. Aspen community types of the Intermountain Region. Ogden, UT: U.S. Department of Agriculture, Forest Service, Intermountain Research Station. (Manuscript in preparation to super- 
sede Mueggler and Campbell 1982, 1986 and Youngblood and Mueggler 1981).

Mueggler, Walter F.; Campbell, Robert B. 1982. Aspen community types on the Caribou and Targee National Forests in southeastern Idaho. Res. Pap. INT-294. Ogden, UT: U.S. Department of Agriculture, Forest Service, Intermountain Forest and Range Experiment Station. $32 \mathrm{p}$.

Mueggler, Walter F.; Campbell, Robert B., Jr. 1986. Aspen community types of Utah. Res. Pap. INT-362. Ogden, UT: U.S. Department of Agriculture, Forest Service, Intermountain Forest and Range Experiment Station. $69 \mathrm{p}$.

Muldavin, Estaben; DeVelice, Robert L.; Dick-Peddie, William. 1986. Forest habitat types of the Prescott, Tonto and western Coronado National Forests, Arizona Final Report. CA 28-K3-307. Fort Collins, CO: U.S. Department of Agriculture, Forest Service, Rocky Mountain Forest and Range Experiment Station. 73 p. plus appendixes.

Pfister, Robert D. 1972. Vegetation and soils in the subalpine forests of Utah. Pullman, WA: Washington State University. Ph.D. dissertation. [Dissertation Abstracts 33(06):98].

Pfister, Robert D.; Kovalchik, Bernard L.; Arno, Stephen F.; Presby, Richard C. 1977. Forest habitat types of Montana. Gen. Tech. Rep. INT-34. Ogden, UT: U.S. Department of Agriculture, Forest Service, Intermountain Forest and Range Experiment Station. 174 p.
Powell, David C. 1987. Aspen community types on the Pike and San Isabel National Forests in south-central Colorado. Denver, CO: U.S. Department of Agriculture, Forest Service, Rocky Mountain Region. (Manuscript in preparation).

Radloff, David L. 1983. Wildland classification with multivariate analyses and remote sensing techniques. Fort Collins, CO: Colorado State University. Ph.D. dissertation. [Dissertation Abstracts 44(04): 116.]

Steele, Robert; Cooper, Stephen V.; Ondov, David M.; Roberts, David W.; Pfister, Robert D. 1983. Forest habitat types of eastern Idaho-western Wyoming. Gen. Tech. Rep. INT-144. Ogden, UT: U.S. Department of Agriculture, Forest Service, Intermountain Forest and Range Experiment Station. 122 p.

Steele, Robert; Pfister, Robert D.; Ryker, Russell A.; Kittams, Jay A. 1981. Forest habitat types of central Idaho. Gen. Tech. Rep. INT-114. Ogden, UT: U.S. Department of Agriculture, Forest Service, Intermountain Forest and Range Experiment Station. 138 p.

Youngblood, Andrew P.; Mauk, Ronald L. 1985. Coniferous forest habitat types of central and southern Utah. Gen. Tech. Rep. INT-187. Ogden, UT: U.S. Department of Agriculture, Forest Service, Intermountain Experiment Station. 89 p.

Youngblood, Andrew P.; Mueggler, Walter F. 1981. Aspen community types on the Bridger-Teton National Forest in western Wyoming. Res. Pap. INT-272. Ogden, UT: U.S. Department of Agriculture, Forest Service, Intermountain Forest and Range Experiment Station. 34 p. 

Table A1. Forest habilat lypos and community lypes in the Rocky Mountains.

\begin{tabular}{|c|c|c|c|c|c|c|}
\hline $\begin{array}{l}\text { Hablint typo or } \\
\text { community typo }\end{array}$ & $\begin{array}{l}\text { Location and } \\
\text { elovallon (faol) }\end{array}$ & sito & $\begin{array}{l}\text { Successlonat } \\
\text { status }\end{array}$ & $\begin{array}{c}\text { Trao } \\
\text { essoclatos }\end{array}$ & $\begin{array}{l}\text { Pinclpat undergrowth } \\
\text { spocias }\end{array}$ & Authorily \\
\hline \multicolumn{7}{|c|}{ Pinus leiophylla series } \\
\hline $\begin{array}{l}\text { Pinus lelophyltal } \\
\text { Aralostaphylos pungens C.T. }\end{array}$ & $\begin{array}{l}\text { Mountains of } \\
\text { south.contral } \\
\text { Arizons } \\
(5.200 \cdot 7,100)\end{array}$ & $\begin{array}{l}\text { Hol vory } \\
\text { dry }\end{array}$ & $\begin{array}{l}\text { P. lalophylla } \\
\text { probably cilmax }\end{array}$ & Junlperus deppaana & $\begin{array}{l}\text { A. pungens } \\
\text { Quercus spp. }\end{array}$ & $\begin{array}{l}\text { Muldavin at al. } \\
1888\end{array}$ \\
\hline $\begin{array}{l}\text { Pinus lelophyllal } \\
\text { Ouercus arlzonica H.T. }\end{array}$ & $\begin{array}{l}\text { Mountains of } \\
\text { soulh.central } \\
\text { Arizona } \\
(4,900 \cdot 7,100)\end{array}$ & $\begin{array}{l}\text { Hot very } \\
\text { dry }\end{array}$ & $\begin{array}{l}\text { P. lalophylla } \\
\text { climax or } \\
\text { co.climax wilh } \\
\text { Pinus ponderosa } \\
\text { Pinus discolor } \\
\text { J. deppoana }\end{array}$ & $\begin{array}{l}\text { P. pondarosa } \\
\text { P. discolor } \\
\text { J. deppeana }\end{array}$ & $\begin{array}{l}\text { O. arizonica } \\
\text { Arctostaphylos spp. } \\
\text { Ouarcus hypolaucoldes } \\
\text { Rhus aromalica } \\
\text { Muhlanbergla longiligute }\end{array}$ & $\begin{array}{l}\text { DeValice and } \\
\text { Ludwlg } 1883 \\
\text { Muidavin ot a1. } \\
1988\end{array}$ \\
\hline $\begin{array}{l}\text { Pinus lelophy/lal } \\
\text { Ouercus emoryl H.T. }\end{array}$ & $\begin{array}{l}\text { Mounialns of } \\
\text { south.central } \\
\text { Arizona } \\
(4,900 \cdot 6,500)\end{array}$ & $\begin{array}{l}\text { Hot vory } \\
\text { dry }\end{array}$ & $\begin{array}{l}\text { P. lelophylla } \\
\text { ciimax of } \\
\text { co-cllmax wilh } \\
\text { P. dlscolor } \\
\text { J. deppeana }\end{array}$ & $\begin{array}{l}\text { P. discolor } \\
\text { J. deppeana }\end{array}$ & $\begin{array}{l}\text { O. emoryl } \\
\text { O. arlzonica } \\
\text { Arlstida orcutilana } \\
\text { Muhlenbergla spp. }\end{array}$ & $\begin{array}{l}\text { Muldavin el al. } \\
1986\end{array}$ \\
\hline $\begin{array}{l}\text { Pinus lelophyllal } \\
\text { Ouercus hypoleucoldes H.T. }\end{array}$ & $\begin{array}{l}\text { Mounlains ol } \\
\text { soulh.conlrai } \\
\text { Arizons } \\
(5,600 \cdot 7,100)\end{array}$ & Hol dry & $\begin{array}{l}\text { P. Jolophylla } \\
\text { climax or } \\
\text { co.climax wilh } \\
\text { P. discolor } \\
\text { J. deppeana }\end{array}$ & $\begin{array}{l}\text { P. discolor } \\
\text { J. deppeana }\end{array}$ & $\begin{array}{l}\text { O. hypolaucoldes } \\
\text { O. arizonica } \\
\text { Munionberg/a spp. }\end{array}$ & $\begin{array}{l}\text { Develice and } \\
\text { Ludwig } 1983 \\
\text { Muldavln el al. } \\
1986\end{array}$ \\
\hline $\begin{array}{l}\text { PInus lelophyllal } \\
\text { Quercus toumeyl H.T. }\end{array}$ & $\begin{array}{l}\text { Mountains ol } \\
\text { south.central } \\
\text { Arizona } \\
(5,500 \cdot 6,500)\end{array}$ & $\begin{array}{l}\text { Hol very } \\
\text { dry }\end{array}$ & $\begin{array}{l}\text { P. lelophylla } \\
\text { cilmax or } \\
\text { co.cllmax wlih } \\
\text { P. discolor. } \\
\text { J. deppeana } \\
\text { minor cllmax }\end{array}$ & $\begin{array}{l}\text { P. discolor } \\
\text { J. deppeana }\end{array}$ & $\begin{array}{l}\text { O. toumeyl } \\
\text { A. pungens }\end{array}$ & $\begin{array}{l}\text { Devellce and } \\
\text { Ludwig } 1983\end{array}$ \\
\hline $\begin{array}{l}\text { PInus lelophyllel } \\
\text { Plplochsetlum limbrialum H.T. } \\
\text { (Seml-flparlan lorest) }\end{array}$ & $\begin{array}{l}\text { Mountalns of } \\
\text { south.cenlral } \\
\text { Artzona } \\
(5.000-6,000)\end{array}$ & Hol molst & $\begin{array}{l}\text { P. lelophylla } \\
\text { climax or } \\
\text { co.climax wlith } \\
\text { P. d/scolor } \\
\text { J. doppeona }\end{array}$ & $\begin{array}{l}\text { Pinus engolmannll } \\
\text { P. discolor } \\
\text { Cupressus arizonica } \\
\text { J. deppeana } \\
\text { Juniparus erythrocarpa }\end{array}$ & $\begin{array}{l}\text { P. llmbrlatum } \\
\text { Juglans major } \\
\text { Prunus serollina } \\
\text { Q. arlzonica } \\
\text { O. hypoleucoldes }\end{array}$ & $\begin{array}{l}\text { Devellce and } \\
\text { Ludwlg } 1983 \\
\text { Muldavin el al. } \\
1986\end{array}$ \\
\hline \multicolumn{7}{|c|}{ Pinus engelmonnii series } \\
\hline $\begin{array}{l}\text { PInus engelmannIll } \\
\text { Quercus arlzonica H.T. }\end{array}$ & $\begin{array}{l}\text { Mountains of } \\
\text { souln.cenlral } \\
\text { Arizona } \\
(6,000-6,500)\end{array}$ & $\begin{array}{l}\text { Warm very } \\
\text { dry }\end{array}$ & $\begin{array}{l}\text { P. engelmannll } \\
\text { cllmax or } \\
\text { co-climax wilh } \\
\text { J. deppeana. } \\
\text { P. discolor } \\
\text { minor cllmax }\end{array}$ & $\begin{array}{l}\text { P. lelophylla } \\
\text { P. discolor } \\
\text { J. deppeana }\end{array}$ & $\begin{array}{l}\text { Q. arizonica } \\
\text { M. longlilgula }\end{array}$ & $\begin{array}{l}\text { Develice and } \\
\text { Ludwig } 1983\end{array}$ \\
\hline $\begin{array}{l}\text { Pinus engelmennill } \\
\text { Ouercus emoryl H.T. }\end{array}$ & $\begin{array}{l}\text { Mountains ol } \\
\text { southarn Arlzona } \\
(5,500 \cdot 6,000)\end{array}$ & $\begin{array}{l}\text { Warm very } \\
\text { ory }\end{array}$ & $\begin{array}{l}\text { P. engelmannil } \\
\text { climax }\end{array}$ & $\begin{array}{l}\text { P. lelophylla } \\
\text { J. deppeane }\end{array}$ & $\begin{array}{l}\text { Q. emoryl } \\
\text { Muhlenbergla emersloyl } \\
\text { M. longillgula }\end{array}$ & $\begin{array}{l}\text { Muldavin al al. } \\
\qquad 1986\end{array}$ \\
\hline $\begin{array}{l}\text { Pinus engelmannill' } \\
\text { Quercus hypoloucoides H.T. }\end{array}$ & $\begin{array}{l}\text { Mountains of } \\
\text { south-centrat } \\
\text { Arizona } \\
(5,800 \cdot 7,100)\end{array}$ & Warm dry & $\begin{array}{l}\text { P. engelmannll } \\
\text { crimax. } \\
\text { P. discolor } \\
\text { P. depocana } \\
\text { minor cllmaxes }\end{array}$ & $\begin{array}{l}\text { P. lolophylla } \\
\text { P. d/scolor } \\
\text { J. deppeana }\end{array}$ & $\begin{array}{l}\text { O. hypolaucoldes } \\
\text { O. arlzon/ca } \\
\text { M. longlllgula }\end{array}$ & $\begin{array}{l}\text { Dovellce and } \\
\text { Ludwig } 1983 \\
\text { Muldavin et at. } \\
\text { T986 }\end{array}$ \\
\hline 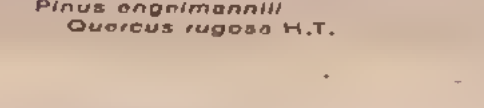 & $\begin{array}{l}\text { Mountains of } \\
\text { souln-central } \\
\text { Arizons } \\
\text { t7.700.n.oms }\end{array}$ & $\begin{array}{l}\text { Warm ory } \\
\text { to woll. } \\
\text { dratnod }\end{array}$ & $\begin{array}{l}\text { P. ongo/mannll } \\
\text { climax }\end{array}$ & $\begin{array}{l}\text { P. Ioloofiyfta } \\
\text { Jopocana }\end{array}$ & $\begin{array}{l}\text { O. rugosa } \\
\text { M. longliliguta }\end{array}$ & $\begin{array}{l}\text { Muldavin et al. } \\
1986\end{array}$ \\
\hline $\begin{array}{l}\text { PInus engelmannill } \\
\text { Muhlenberglo longligulo H.T. }\end{array}$ & $\begin{array}{l}\text { Mountains ol } \\
\text { soulh.cenlral } \\
\text { Arizona } \\
(6,500 \cdot 7,000)\end{array}$ & Warm ory & $\begin{array}{l}\text { P. engelmannil } \\
\text { climax. } \\
\text { P. discolor } \\
\text { J. deppeone } \\
\text { minor cllmaxes }\end{array}$ & $\begin{array}{l}\text { P. discolor } \\
\text { J. deppeane }\end{array}$ & $\begin{array}{l}\text { M. longlligula } \\
\text { O. gambelll } \\
\text { O. hypoleucoldes }\end{array}$ & $\begin{array}{l}\text { Devellce and } \\
\text { Ludwlg } 1983\end{array}$ \\
\hline \multicolumn{7}{|c|}{ Pinus ponderosa series } \\
\hline $\begin{array}{l}\text { Pinus ponderosal } \\
\text { Arclostaphylos pelula H.T. }\end{array}$ & $\begin{array}{l}\text { Mounlalns of } \\
\text { southern Utah } \\
\text { and weslarn } \\
\text { Colorado } \\
(7,500-8,500)\end{array}$ & $\begin{array}{l}\text { Warm very } \\
\text { ory }\end{array}$ & $\begin{array}{l}\text { P. ponderosa } \\
\text { climax }\end{array}$ & $\begin{array}{l}\text { PInus llexilis } \\
\text { Juniperus scopulorum }\end{array}$ & $\begin{array}{l}\text { A. palula } \\
\text { Berberls repens } \\
\text { Quercus gambelll } \\
\text { Purshia Iridentala } \\
\text { Carex ross"l }\end{array}$ & $\begin{array}{l}\text { Hoffman } 1988 \\
\text { Youngblood and } \\
\text { Mauk } 1985\end{array}$ \\
\hline $\begin{array}{l}\text { PInus ponderosal } \\
\text { Arclostaphylos pungens C.T. } \\
\text { [P. ponderosal } \\
\text { Arclostaphylos spp. C.T.] } \\
\text { [P. ponderosalMlxed chapparel } \\
\text { C.T.] }\end{array}$ & $\begin{array}{l}\text { Mounlains of } \\
\text { Arlzona } \\
(5,000 \cdot 7,600)\end{array}$ & $\begin{array}{l}\text { Warm vary } \\
\text { dry }\end{array}$ & $\begin{array}{l}\text { P. ponderosa } \\
\text { cllmax or } \\
\text { co.cllmax wilh } \\
\text { J. deppeana }\end{array}$ & $\begin{array}{l}\text { P. edulls } \\
\text { J. deppeana }\end{array}$ & $\begin{array}{l}\text { A. pungens } \\
\text { Arclostaphylos spp. } \\
\text { Cercocarpus montanus } \\
\text { Quercus spp. } \\
\text { Bouteloue gracllis } \\
\text { Muhlenbergle virescens }\end{array}$ & $\begin{array}{l}\text { Fllzhugh el al. } \\
1987 \\
\text { Hanks ef al. } \\
1983 \\
\text { Muldavin el al. } \\
1986\end{array}$ \\
\hline $\begin{array}{l}\text { Pinus ponderosal } \\
\text { Arclosiophylos uve.urs/ H.T. }\end{array}$ & $\begin{array}{l}\text { Biack HIIls and } \\
\text { Bear Lodge } \\
\text { Mounlalns, Soulh } \\
\text { Dakota and easlern } \\
\text { Wyoming }(5,100 \text {. } \\
6,700) \text {; mountains } \\
\text { of soulheaslarn } \\
\text { Wyoming }(8,300- \\
8,300) \text {, southern } \\
\text { Colorado, and } \\
\text { northarn New Mexico } \\
(7,700-9,200)\end{array}$ & $\begin{array}{l}\text { Warm vary } \\
\text { dry }\end{array}$ & $\begin{array}{l}\text { P. ponderosa } \\
\text { climax. } \\
\text { P. menzles / } \\
\text { may be minor } \\
\text { cllmax (CQ,NM) }\end{array}$ & $\begin{array}{l}\text { Usually pura slands } \\
\text { (SD) } \\
\text { P. Ilexills } \\
\text { Populus tremuloldes } \\
\text { (WY) } \\
\text { Pseudotsuga menzlesil } \\
\text { (CO,NM) }\end{array}$ & $\begin{array}{l}\text { A. uva-ursl } \\
\text { Symphoricarpos albus } \\
\text { Festuca arizonica } \\
\text { Muhlenbergla moniana } \\
\text { Carex spp. } \\
\text { Arnica cordllolla } \\
\text { Lathyrus ochroloucus } \\
\text { Luplnus argenteus }\end{array}$ & $\begin{array}{l}\text { Alexander el al. } \\
1986 \\
\text { DeValice el al. } \\
1986 \\
\text { Hoffman and } \\
\text { Alexander } 1987\end{array}$ \\
\hline $\begin{array}{l}\text { PInus ponderosal } \\
\text { Artem/sla nova H.T. } \\
\text { [P. Ponderosal } \\
\text { Artemisla arbuscula H.T.] }\end{array}$ & $\begin{array}{l}\text { Mounlains ol } \\
\text { soulhern Ulah } \\
(8,000-9,000) \\
\text { northern Now } \\
\text { Mexico, and } \\
\text { southern Colorado } \\
(8,000-8,200)\end{array}$ & $\begin{array}{l}\text { Warm very } \\
\text { dry }\end{array}$ & $\begin{array}{l}\text { P. ponderosa } \\
\text { climax (UT) or } \\
\text { co.cllmax wlih } \\
\text { P. edulls } \\
\text { J. scopulorum } \\
(\text { CO,NM) }\end{array}$ & $\begin{array}{l}\text { P. edulls } \\
\text { P. Hexll/s (UT) } \\
\text { J. scopulorum }\end{array}$ & $\begin{array}{l}\text { A. nova } \\
\text { A. arbuscula } \\
\text { Chrysothamnus } \\
\text { viscidllorus } \\
\text { O. gambelli } \\
\text { relradymla canescens } \\
\text { B. gracllls }\end{array}$ & $\begin{array}{l}\text { Devellce el al. } \\
1986 \\
\text { Youngblood and } \\
\text { Mauk } 1985\end{array}$ \\
\hline $\begin{array}{l}\text { Pinus ponderosal } \\
\text { Cercocarpus ledilollus H.T. }\end{array}$ & $\begin{array}{l}\text { Mounlalns of } \\
\text { canlral and } \\
\text { soulhern Ulah } \\
(6,800 \cdot 8,100)\end{array}$ & $\begin{array}{l}\text { Warm very } \\
\text { dry }\end{array}$ & $\begin{array}{l}\text { P. ponderosa } \\
\text { cllmax. } \\
\text { J. scopulorum } \\
\text { minor climax }\end{array}$ & $\begin{array}{l}\text { P. edulls } \\
\text { J. scopulorum }\end{array}$ & $\begin{array}{l}\text { C. ledilollus } \\
\text { A. Iridentala } \\
\text { Juniperus spp. } \\
\text { Q. gambelli } \\
\text { Symphoricarpos oreophilus }\end{array}$ & $\begin{array}{l}\text { Youngblood and } \\
\text { Mauk } 1985\end{array}$ \\
\hline $\begin{array}{l}\text { Plnus ponderosal } \\
\text { Cercocarpus montanus H.T. } \\
{[P \text {. ponderoselC. monianus. }} \\
\text { Ahus Irllobela H.T.] }\end{array}$ & $\begin{array}{l}\text { Fronl Range, } \\
\text { norlh.central } \\
\text { Colorado } \\
(6,300 \cdot 7,000)\end{array}$ & $\begin{array}{l}\text { Warm vary } \\
\text { dry }\end{array}$ & $\begin{array}{l}\text { P. ponderosa } \\
\text { climax }\end{array}$ & $\begin{array}{l}\text { Usually pure sfands. } \\
\text { May contaln } \\
\text { P. menzles }\end{array}$ & $\begin{array}{l}\text { C. monienus } \\
\text { Opunile polyecanthe } \\
\text { A. trllobela } \\
\text { C. rossli } \\
\text { Arlemisla Irlgida } \\
\text { Geranlum Iremontl }\end{array}$ & $\begin{array}{l}\text { Hass and } \\
\text { Alexander } 1986 \\
\text { Radiofl } 1983\end{array}$ \\
\hline $\begin{array}{l}\text { Plnus ponderosal } \\
\text { Cowanla mexicana C.T. }\end{array}$ & $\begin{array}{l}\text { Mounlalns of } \\
\text { norlhern Arizona } \\
(8,700 \cdot 7,500)\end{array}$ & $\begin{array}{l}\text { Warm very } \\
\text { dry }\end{array}$ & $\begin{array}{l}\text { P. ponderosa } \\
\text { climax }\end{array}$ & $\begin{array}{l}\text { Usually pura slands. } \\
\text { May contain } \\
\text { P. adulis } \\
\text { J. scopulorum }\end{array}$ & $\begin{array}{l}\text { C. mexicana } \\
\text { B. gracills } \\
\text { M. montena } \\
\text { Sitanion hysirix }\end{array}$ & Hanks of al. 1983 \\
\hline $\begin{array}{l}\text { Pinus pondarosal } \\
\text { Juglans major H.T. } \\
\text { (Seml-rlparian forasi) }\end{array}$ & $\begin{array}{l}\text { Mounlains of } \\
\text { south.central } \\
\text { Arizona } \\
(5,500 \cdot 8,500)\end{array}$ & Warm mols1 & $\begin{array}{l}\text { P. ponderosa } \\
\text { cllmax }\end{array}$ & Usually pure stands & $\begin{array}{l}\text { J. malor } \\
\text { Agropyron spp. } \\
\text { Panicum bulbosum } \\
\text { Poo pratensis }\end{array}$ & $\begin{array}{l}\text { Muldavin of al. } \\
1986\end{array}$ \\
\hline
\end{tabular}





\begin{tabular}{|c|c|c|c|c|c|c|}
\hline $\begin{array}{l}\text { Habltat type or } \\
\text { communlly typo }\end{array}$ & $\begin{array}{l}\text { Locatlon and } \\
\text { olovatlon (faet) }\end{array}$ & Slte & $\begin{array}{l}\text { Successlonel } \\
\text { stetus }\end{array}$ & $\begin{array}{c}\text { Trae } \\
\text { essocletes }\end{array}$ & $\begin{array}{l}\text { Princlpal undergrowth } \\
\text { opecles }\end{array}$ & Authorily \\
\hline $\begin{array}{l}\text { Pinus ponderosel } \\
\text { Juniperus communis H.T. }\end{array}$ & $\begin{array}{l}\text { Blghorn Mounleins, } \\
\text { norlh.cenlral } \\
\text { Wyoming: Black } \\
\text { HIIls end Beer Lodge } \\
\text { Mountelns, Soulh } \\
\text { Dekote and eastern } \\
\text { Wyoming: mountelns of } \\
\text { southwestern North } \\
\text { Dekola end south. } \\
\text { e日stern Montene } \\
(4,000 \cdot 6,300)\end{array}$ & $\begin{array}{l}\text { Werm dry } \\
\text { to well. } \\
\text { drelned }\end{array}$ & $\begin{array}{l}\text { P. ponderose } \\
\text { climex }\end{array}$ & $\begin{array}{l}\text { Usuelly pure slends. } \\
\text { Mey conteln } \\
\text { O. Iremuloldes } \\
\text { Ouercus mocrocerpe } \\
\text { (tree andlor shrub) }\end{array}$ & $\begin{array}{l}\text { J. communis } \\
\text { B. repens } \\
\text { Splreee belulliolio } \\
\text { S. elbus } \\
\text { Hesperochloa kingli } \\
\text { P. pratensis } \\
\text { Asiregelus miser } \\
\text { Clemalls tenulloba }\end{array}$ & $\begin{array}{l}\text { Hansen end } \\
\text { Holfman } 1988 \\
\text { Hoffmen and } \\
\text { Alexander } 1987\end{array}$ \\
\hline $\begin{array}{l}\text { Pinus ponderose. } \\
\text { Juniperus scopulorum H.T. } \\
\text { [P. ponderosed. scopulorumi } \\
\text { Bouteloue curtipendule H.T.] }\end{array}$ & $\begin{array}{l}\text { Bleck Hllis and } \\
\text { Beer Lodge } \\
\text { Mountelns, South } \\
\text { Dekole and } \\
\text { eastern Wyoming } \\
(3,800 \cdot 4,000)\end{array}$ & $\begin{array}{l}\text { Warm dry } \\
10 \text { well. } \\
\text { dreined }\end{array}$ & $\begin{array}{l}\text { p. ponderose } \\
\text { cllmex lo } \\
\text { co.cllmax wilh } \\
\text { J. scopulorum }\end{array}$ & J. scopulorum & $\begin{array}{l}\text { 8. curlipendule } \\
\text { Oryzopsis micrenthe } \\
\text { Anemone patens } \\
\text { A. Iriglde } \\
\text { Compenule rolundilolle }\end{array}$ & $\begin{array}{l}\text { Hofiman and } \\
\text { Alexander } 1987\end{array}$ \\
\hline $\begin{array}{l}\text { Pinus ponderosal } \\
\text { Physocerpus malveceus H.T. }\end{array}$ & $\begin{array}{l}\text { Mounleins ol } \\
\text { northern and } \\
\text { central ldaho and } \\
\text { eastern Washinglon } \\
(<3,000)\end{array}$ & Warm dry & $\begin{array}{l}\text { P. ponderose } \\
\text { cllmex }\end{array}$ & Usually pure slands & $\begin{array}{l}\text { P. malveceus } \\
\text { Coanothus senguineus } \\
\text { Holodiscus discolor } \\
\text { Erylhronium grendifiorum } \\
\text { Gellum boreele }\end{array}$ & $\begin{array}{l}\text { Cooper et al. } 1987 \\
\text { Daubenmire and } \\
\text { Daubenmire } 1968 \\
\text { Steele et al. } 1981\end{array}$ \\
\hline $\begin{array}{l}\text { Pinus ponderosel } \\
\text { Physocerpus monogynus H.T. }\end{array}$ & $\begin{array}{l}\text { Blghorn Mounlains, } \\
\text { north-central } \\
\text { Wyoming }(6,100 \text {. } \\
6,600) ; \text { Black HIIIs } \\
\text { and Beer Lodge } \\
\text { Mountains, South } \\
\text { Dakota and } \\
\text { eastern Wyoming } \\
(5,100.5,700)\end{array}$ & $\begin{array}{l}\text { Werm dry } \\
\text { lo well. } \\
\text { drelned }\end{array}$ & $\begin{array}{l}\text { P. ponderosa } \\
\text { climax }\end{array}$ & $\begin{array}{l}\text { Usually pure stends. } \\
\text { May conlain } \\
J . \text { scopulorum }\end{array}$ & $\begin{array}{l}\text { P. monogynus } \\
\text { B. repens } \\
\text { S. belulliolla } \\
\text { S. elbus } \\
\text { Cystoplerls lragllis } \\
\text { G. boreole } \\
\text { Solldago speciose }\end{array}$ & $\begin{array}{l}\text { Hoffman and } \\
\text { Alexander } 1978 \\
1987\end{array}$ \\
\hline $\begin{array}{ll}\text { Pinus ponderosel } & M \\
\text { Prunus virginiene H.T.(MT,ND); } & \text { e日 } \\
\text { C.T.(SD) } & \text { so } \\
\text { P. virginlene (IyplC) phase } & \text { No } \\
\text { Shepherdie canadensis phase (MT) } & \text { no } \\
& \text { So } \\
& \text { i }\end{array}$ & $\begin{array}{l}\text { Mountains of south. } \\
\text { e日stern Monlans, } \\
\text { soulhwestern } \\
\text { North Dakota, and } \\
\text { northern Black Hills, } \\
\text { Soulh Oakole } \\
(3,500-4,300)\end{array}$ & $\begin{array}{l}\text { Warm molst } \\
\text { to well. } \\
\text { drained }\end{array}$ & $\begin{array}{l}\text { P. ponderosa } \\
\text { cllmax }\end{array}$ & Usually pure stands & $\begin{array}{l}\text { P. virginiana } \\
\text { Amelenchler elnilolle } \\
\text { B. repens } \\
\text { S. canedensis } \\
\text { S. elbus } \\
\text { Arnica cordllolla } \\
\text { C. Iragllis }\end{array}$ & $\begin{array}{l}\text { Hansen and } \\
\text { Holfman } 1988 \\
\text { Hoffman and } \\
\text { Alexander } 1987 \\
\text { Pfisler et al. } 1977\end{array}$ \\
\hline 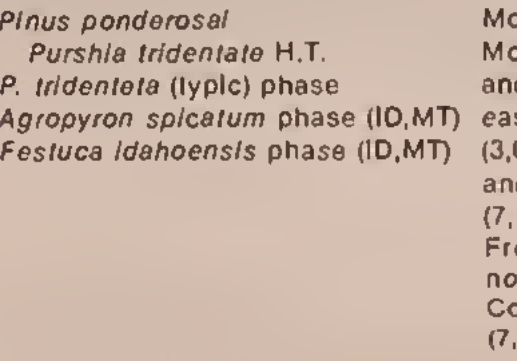 & $\begin{array}{l}\text { Mountains of } \\
\text { Monlana, northern } \\
\text { and central Idaho, } \\
\text { eastern Washinglon } \\
(3,000-6,000) \text {, } \\
\text { and southern Utah } \\
(7,100-9,000) \text {; } \\
\text { Front Range of } \\
\text { north-centrat } \\
\text { Colorado } \\
(7,600-8,700)\end{array}$ & Warm dry & $\begin{array}{l}\text { P. ponderosa } \\
\text { cllmax. } \\
\text { J. scopulorum } \\
\text { minor climax }\end{array}$ & $\begin{array}{l}\text { P. menzles II } \\
\text { J. scopulorum }\end{array}$ & $\begin{array}{l}\text { P. Iridenlata } \\
\text { A. trldentate } \\
\text { P. virginlana } \\
\text { A. splcalum } \\
\text { Aristida longisela } \\
\text { F. ldaoensis } \\
\text { M. montana } \\
\text { C. rossil } \\
\text { Belsemornize seglitere }\end{array}$ & $\begin{array}{l}\text { Oaubenmire and } \\
\text { Daubenmire } 1968 \\
\text { Hess and } \\
\text { Alexander } 1986 \\
\text { Pflster et al. } 1977 \\
\text { Sleele et al. } 1981 \\
\text { Youngblood and } \\
\text { Mauk } 1985\end{array}$ \\
\hline 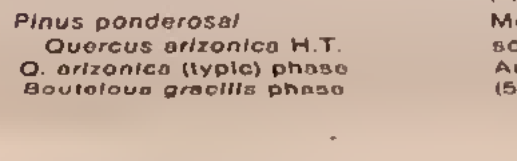 & $\begin{array}{l}\text { Mountains of } \\
\text { south.contral } \\
\text { Arizonn } \\
\text { (5.500.7.80) }\end{array}$ & Hol dry & 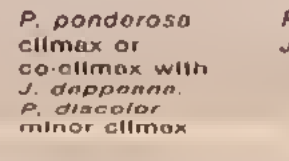 & $\begin{array}{l}\text { P. discolor } \\
\text { J. doppoans }\end{array}$ & $\begin{array}{l}\text { O. arizonica } \\
\text { Coanofhus londlorl } \\
\text { Ouarcus spp. } \\
\text { B. grabellis } \\
\text { M. Ionglilgula }\end{array}$ & $\begin{array}{l}\text { Devellce and } \\
\text { Ludwig } 1983 \\
\text { Muldovin or al. } \\
\text { 1986 }\end{array}$ \\
\hline $\begin{array}{l}\text { Pinus ponderosal } \\
\text { Ouercus emoryl H.T. }\end{array}$ & $\begin{array}{l}\text { Mountalns of } \\
\text { south.cenlral } \\
\text { Arizona } \\
(5,300 \cdot 6,900)\end{array}$ & Warm dry & $\begin{array}{l}\text { p. ponderoso } \\
\text { cllmax }\end{array}$ & J. deppeane & $\begin{array}{l}\text { O. emoryi } \\
\text { Arclostephylos spp. } \\
\text { C. fendlerl } \\
\text { Gerrya wrighlli } \\
\text { O. arizonica }\end{array}$ & $\begin{array}{l}\text { Muldavln et al. } \\
\quad 1986\end{array}$ \\
\hline 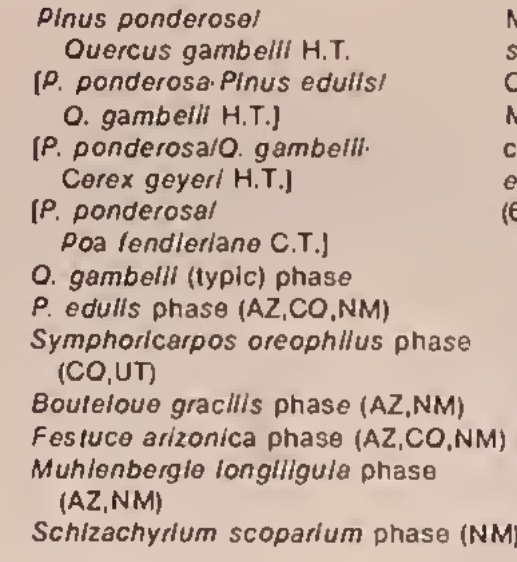 & $\begin{array}{l}\text { Mountalns of } \\
\text { soulhern Ulah, } \\
\text { Colorado, New } \\
\text { Mexico, and soulh. } \\
\text { central and } \\
\text { easlern Arizona } \\
(6,500 \cdot 9,200)\end{array}$ & Warm dry & $\begin{array}{l}\text { P. ponderosa } \\
\text { climax or } \\
\text { co.cllmex with } \\
\text { P. edulls. } \\
\text { Pinus engelmannil } \\
\text { J. deppeane } \\
\text { Juniperus } \\
\text { monospermo } \\
\text { J. scopulorum } \\
\text { minor cllmaxes }\end{array}$ & $\begin{array}{l}\text { P. edulls } \\
\text { P. menzles ll } \\
\text { P. discolor } \\
\text { P. engelmennll } \\
\text { J. deppoana } \\
\text { J. monosperma } \\
\text { Juniperus osteosperme } \\
\text { J. scopulorum }\end{array}$ & $\begin{array}{l}\text { O. gambelll } \\
\text { B. repens } \\
\text { J. communis } \\
\text { Rosa woodsll } \\
\text { S. oreophllus } \\
\text { B. gracllls } \\
\text { F. arlzonica } \\
\text { Koelerle cristata } \\
\text { (K. macrantha) } \\
\text { Muhlenbergle spp. } \\
\text { P. fendleriona } \\
\text { S. scoparium } \\
\text { C. geyerl } \\
\text { Achlleo lanulosa }\end{array}$ & $\begin{array}{l}\text { Alexander et al. } \\
\text { 1984a. } 1987 \\
\text { DeVelice el al. } \\
1986 \\
\text { DeVelice and } \\
\text { Ludwig 1983 } \\
\text { Fllzhugh et al. } \\
1987 \\
\text { Hanks et al. } 1983 \\
\text { Hess and Wasser } \\
1982 \\
\text { Hoffman } 1988 \\
\text { Muldavin et al. } \\
1986 \\
\text { Youngblood and } \\
\text { Mauk 1985 }\end{array}$ \\
\hline $\begin{array}{l}\text { Pinus ponderosal } \\
\text { Ouercus griseo H.T. } \\
\text { Q. grisea (typic) phese } \\
\text { Muhlenbergla longillgula phase } \\
\text { Muhlenbergle montena phase }\end{array}$ & $\begin{array}{l}\text { Mountains of } \\
\text { soulhwestern New } \\
\text { Mexlco and } \\
\text { eastern Arizona } \\
(6,100 \cdot 8,800)\end{array}$ & Warm dry & $\begin{array}{l}\text { P. ponderose } \\
\text { climex of } \\
\text { co.climax with } \\
\text { P. odulis } \\
\text { J. deppeene } \\
\text { J. monosperme. } \\
\text { P. menzies } \\
\text { minor cllmax }\end{array}$ & $\begin{array}{l}\text { P. edulls } \\
\text { P. menzleshl } \\
\text { J. deppeana } \\
\text { J. monosperme }\end{array}$ & $\begin{array}{l}\text { O. grisea } \\
\text { B. gracillis } \\
\text { M. longlligula } \\
\text { M. montana } \\
\text { M. virescens } \\
\text { P. Iendieriane }\end{array}$ & $\begin{array}{l}\text { Fltzhugh ot al. } \\
\quad 1987\end{array}$ \\
\hline $\begin{array}{l}\text { Pinus ponderosel } \\
\text { Ouercus hypoleucoides H.T. }\end{array}$ & $\begin{array}{l}\text { Mountalns of } \\
\text { south.ceniral } \\
\text { Afizona } \\
(5,700 \cdot 8,000)\end{array}$ & Warm dry & $\begin{array}{l}\text { P. pondarosa } \\
\text { climex. } \\
\text { P. discolor } \\
\text { Pinus ongeimennil } \\
\text { J. deppoene } \\
\text { minor climaxes }\end{array}$ & $\begin{array}{l}\text { P. engeimennil } \\
\text { P. lelophylla } \\
\text { P. discolor } \\
\text { J. deppeane }\end{array}$ & $\begin{array}{l}\text { Q. hypoleucoldes } \\
\text { C. tendlerl } \\
\text { G. wrighlli } \\
\text { Ouercus } \$ p p \text {. } \\
\text { M. longliguite } \\
\text { D. tendleriana }\end{array}$ & $\begin{array}{l}\text { Devellce and } \\
\text { Ludwig } 1983 \\
\text { Muldavin et al. } \\
1986\end{array}$ \\
\hline $\begin{array}{l}\text { Pinus ponderosal } \\
\text { Ouercus mecrocarpe H.T. }\end{array}$ & $\begin{array}{l}\text { Black HIIIs and } \\
\text { Bear Lodgo } \\
\text { Mountalns, Soulh } \\
\text { Dakote and eastern } \\
\text { Wyoming }(4,100 \cdot 5,300)\end{array}$ & $\begin{array}{l}\text { Warm dry } \\
\text { lo well. } \\
\text { drained }\end{array}$ & $\begin{array}{l}\text { P. ponderosa } \\
\text { cllmax }\end{array}$ & Usually pure stands & $\begin{array}{l}\text { O. mecrocerpe } \\
\text { A. einilolle } \\
\text { B. repens } \\
\text { Ostrya virginiena } \\
\text { Elymus virginicus }\end{array}$ & $\begin{array}{l}\text { Hollman and } \\
\text { Alexander } 1987\end{array}$ \\
\hline $\begin{array}{l}\text { Pinus ponderosel } \\
\text { Ouercus rugosa H.T. }\end{array}$ & $\begin{array}{l}\text { Mounlalns ol } \\
\text { soulh central } \\
\text { Arlzona } \\
(7,000 \cdot 8,800)\end{array}$ & $\begin{array}{l}\text { Werm molst } \\
\text { to well. } \\
\text { dralned }\end{array}$ & $\begin{array}{l}\text { P. ponderosa } \\
\text { cllmax }\end{array}$ & $\begin{array}{l}\text { Usually pure stands. } \\
\text { May conteln } \\
\text { Plnus strobllormis } \\
\text { P. menzlesll }\end{array}$ & $\begin{array}{l}\text { O. rugosa } \\
\text { Ouarcus spp. }\end{array}$ & $\begin{array}{l}\text { Devellce and } \\
\text { Ludwig } 1983 \\
\text { Muldavin el el. } \\
1986\end{array}$ \\
\hline $\begin{array}{l}\text { Pinus ponderosal } \\
\text { Ouercus undulata H.T. } \\
\text { Q. undulala (typlc) phase } \\
\text { Muhlenbergla dubla phase (NM) } \\
\text { Muhlenbergla longlligula phase } \\
\text { (NM) }\end{array}$ & $\begin{array}{l}\text { Mounlains of } \\
\text { norlhern and } \\
\text { southern New } \\
\text { Mexlco } \\
(6,500 \cdot 8,000)\end{array}$ & Hot dry & $\begin{array}{l}\text { P. ponderosa } \\
\text { cllmax or } \\
\text { co.cllmax with } \\
\text { P. edulis. } \\
\text { J. doppeana } \\
\text { J. monosperma } \\
\text { J. scopulorum } \\
\text { minor cllmaxes }\end{array}$ & $\begin{array}{l}\text { P. manziesll } \\
\text { P. strobitormis } \\
\text { P. edulls } \\
\text { J. deppeane } \\
\text { J. monospermo } \\
\text { J. scopulorum }\end{array}$ & $\begin{array}{l}\text { O. undulata } \\
\text { Q. arizonice } \\
\text { O. gambelli } \\
\text { Andropogon spp. } \\
\text { Bouteloue spp. } \\
\text { M. dubla } \\
\text { M. longlligula } \\
\text { Artamisio ludoviclana }\end{array}$ & $\begin{array}{l}\text { Alexender et el. } \\
1984 \text { a } \\
\text { Devellce et al. } \\
1986\end{array}$ \\
\hline $\begin{array}{l}\text { Pinus ponderosal } \\
\text { Ribos inermo H.T. } \\
\text { (Scree forest) }\end{array}$ & $\begin{array}{l}\text { Mountains of } \\
\text { norlhern New } \\
\text { Mexico } \\
(7,500 \cdot 8,500)\end{array}$ & Cool dry & $\begin{array}{l}\text { P. ponderosa } \\
\text { climax }\end{array}$ & $\begin{array}{l}\text { P. menzlasil } \\
\text { P. edulis } \\
\text { J. scopulorum } \\
\text { J. deppoana }\end{array}$ & $\begin{array}{l}\text { A. Inerme } \\
\text { Q. gambelll } \\
\text { M. montana } \\
\text { P. Iondierione }\end{array}$ & $\begin{array}{l}\text { DeVellce el al. } \\
1986\end{array}$ \\
\hline
\end{tabular}





\begin{tabular}{|c|c|c|c|c|c|c|}
\hline $\begin{array}{l}\text { Mobltet type or } \\
\text { communlty typo }\end{array}$ & $\begin{array}{l}\text { Locellon end } \\
\text { elevation (feet) }\end{array}$ & Site & $\begin{array}{l}\text { Succoesional } \\
\text { stalus }\end{array}$ & $\begin{array}{l}\text { Treo } \\
\text { assoclstos }\end{array}$ & $\begin{array}{l}\text { Principal undergrowth } \\
\text { spscles }\end{array}$ & Authorliy \\
\hline $\begin{array}{l}\text { PInus ponderosal } \\
\text { Splraee betulliolla H.T. }\end{array}$ & $\begin{array}{l}\text { Blghorn Mountalns, } \\
\text { north.ceniral } \\
\text { Wyoming } \\
(5,600 \cdot 5,900)\end{array}$ & Werm dry & $\begin{array}{l}\text { P. ponderose } \\
\text { cllmex }\end{array}$ & Uevelly pure stands & $\begin{array}{l}\text { S. botulliolle } \\
\text { C. tenulloba } \\
\text { S. albus } \\
\text { F. Idahoens/s } \\
\text { H. kingll } \\
\text { G. borealo }\end{array}$ & $\begin{array}{l}\text { Hoifman and } \\
\text { Alexander } 1976\end{array}$ \\
\hline $\begin{array}{l}\text { Pinus ponderosel } \\
\text { Symphoricarpos albus H.T. } \\
\text { S. elbus (Iyplc) phese } \\
\text { Berberis repens phese (MT) } \\
\text { Oryzopsis esperllolla phese (SD) } \\
\text { Batsamorhiza segillate phase (SD) }\end{array}$ & $\begin{array}{l}\text { Mountains of } \\
\text { eeslorn Weshinglon, } \\
\text { northern and cenlrel } \\
\text { Idaho, and central } \\
\text { and soulheeslern } \\
\text { Monlane }(2,600 \cdot 5,400) ; \\
\text { Bleck HIIIs and } \\
\text { Boer Lodge Mounlalns, } \\
\text { South Dekole and } \\
\text { eastern Wyoming } \\
(4,200-6,000)\end{array}$ & Worm ory & $\begin{array}{l}\text { P. ponderosa } \\
\text { climex }\end{array}$ & $\begin{array}{l}\text { Usuelly pure slends. } \\
\text { May contein } \\
\text { P. tromuloldes } \\
\text { (SD,Wr) }\end{array}$ & $\begin{array}{l}\text { S. albus } \\
\text { B. repens } \\
\text { J. communls } \\
\text { P. virglniana } \\
\text { Rosa spp. } \\
\text { S. canedensls } \\
\text { S. betulliolla } \\
\text { O. asperlfolle } \\
\text { Carox loenee } \\
\text { B. saglltata }\end{array}$ & $\begin{array}{l}\text { Cooper el al. } 1987 \\
\text { Daubenmire and } \\
\text { Daubenmire } 1968 \\
\text { Hoffman and } \\
\text { Alexender } 1987 \\
\text { Plisler el al. } 1977 \\
\text { Steele el al. } 1981\end{array}$ \\
\hline $\begin{array}{l}\text { Pinus ponderosal } \\
\text { Symphoricarpos oreophilus H.T. }\end{array}$ & $\begin{array}{l}\text { Mounteins of } \\
\text { centrel Ideho } \\
(\leq 5,000) \text { end } \\
\text { southern Uteh } \\
(7,900-8,800)\end{array}$ & Warm dry & $\begin{array}{l}\text { P. ponderosa } \\
\text { climax }\end{array}$ & $\begin{array}{l}\text { Usuelly pure slands } \\
\text { (ID). } \\
\text { Mey contein (UT) } \\
\text { J. scopulorum } \\
\text { P. tromuloldes }\end{array}$ & $\begin{array}{l}\text { S. oreophllus } \\
\text { A. alnilolla } \\
\text { B. ropens } \\
\text { P. virgintana } \\
\text { P. tridentata } \\
\text { A. spicatum }\end{array}$ & $\begin{array}{l}\text { Sleele ol al. 1981 } \\
\text { Youngblood and } \\
\text { Mouk } 1985\end{array}$ \\
\hline $\begin{array}{l}\text { PInus ponderosal } \\
\text { Agropyron splcalum H.T. }\end{array}$ & $\begin{array}{l}\text { Mountains of } \\
\text { eastern Weshington, } \\
\text { tdaho, soulheastern } \\
\text { and west-cenlrel } \\
\text { Montene, norlh. } \\
\text { central Wyoming, } \\
\text { and soulhwestern } \\
\text { North Dakota } \\
(2,400-6,000)\end{array}$ & $\begin{array}{l}\text { Hot very } \\
\text { dry }\end{array}$ & $\begin{array}{l}\text { P. ponderoso } \\
\text { cilmax. } \\
\text { J. scopulorum } \\
\text { may be minor } \\
\text { cllmax }\end{array}$ & $\begin{array}{l}\text { Usually pure stands. } \\
\text { May conlain } \\
\text { J. scopulorum }\end{array}$ & $\begin{array}{l}\text { A. splcalum } \\
\text { Arlemisla spp. } \\
\text { A. longisela } \\
\text { Bromus tectorum } \\
\text { F. ldahoenis } \\
\text { Poe spp. } \\
\text { B. saglliala } \\
\text { Lomellum dissectum } \\
\text { Mellca bulbosa }\end{array}$ & $\begin{array}{l}\text { Cooper el el. } 1987 \\
\text { Daubenmire and } \\
\text { Daubenmire } 1988 \\
\text { Hansen and Holfman } \\
\text { 1988 } \\
\text { Hoflman and } \\
\text { Alexander } 1976 \\
\text { Pflsler el al. } 1977 \\
\text { Sleole el al. } 1981\end{array}$ \\
\hline $\begin{array}{l}\text { Pinus ponderosal } \\
\text { Andropogon spp. H.T. }\end{array}$ & $\begin{array}{l}\text { Mountelns of } \\
\text { southeaslern } \\
\text { Monlane } \\
(\leq 4,000)\end{array}$ & $\begin{array}{l}\text { Warm very } \\
\text { dry }\end{array}$ & $\begin{array}{l}\text { P. ponderosa } \\
\text { climax. } \\
\text { J. scopulorum } \\
\text { minor cllmax }\end{array}$ & J. scopulorum & $\begin{array}{l}\text { A. gerardil } \\
\text { A. scoparius }\end{array}$ & Pflster et al. 1977 \\
\hline $\begin{array}{l}\text { Pinus ponderosel } \\
\text { Boutelous gracllls H.T. } \\
\text { 8. gracllis (lyplc) phase } \\
\text { Pinus edulls phase (AZ) } \\
\text { Artemisia tridentela phase (AZ) } \\
\text { Quercus gambelli phase (AZ) } \\
\text { Andropogon halli phase (AZ) } \\
\text { Schizachyrlum scoparlum phase (NN } \\
\text { VIIIs arizonica phase (AZ) }\end{array}$ & $\begin{array}{l}\text { Mounlalns of } \\
\text { Arizone and } \\
\text { New Mexico } \\
(5,700-8,600) \\
\text { M) }\end{array}$ & $\begin{array}{l}\text { Warm very } \\
\text { dry }\end{array}$ & $\begin{array}{l}\text { P. ponderose } \\
\text { cllmax or } \\
\text { co-climax with } \\
\text { P. edulls } \\
\text { P. dlscolor } \\
\text { J. deppeena. } \\
\text { J. monosperme } \\
\text { J. scopulorum } \\
\text { minor climaxes }\end{array}$ & $\begin{array}{l}\text { P. edulls } \\
\text { P. discolor } \\
\text { J. deppeane } \\
\text { J. monosperma } \\
\text { J. scopulorum }\end{array}$ & $\begin{array}{l}\text { B. gracllis } \\
\text { A. tildentela } \\
\text { Q. gambelli } \\
\text { O. grisea } \\
\text { A. hallil } \\
\text { M. longlilgula } \\
\text { P. fendleriana } \\
\text { S. scoparlum } \\
\text { V. erizonica }\end{array}$ & $\begin{array}{l}\text { Alexander et al. } \\
1987 \\
\text { DeVelice et al. } \\
1986 \\
\text { DeVellce and } \\
\text { Ludwlg } 1983 \\
\text { Fitzhugh ot al. } \\
1987 \\
\text { Hanks el al. } \\
1983 \\
\text { Muldavin et al. } \\
1986\end{array}$ \\
\hline 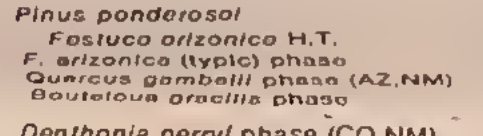 & $\begin{array}{l}\text { Mountains of } \\
\text { Arizona and Now } \\
\text { Mortco } 17.200 . \\
9.500 \text {. Front } \\
\text { Ranos and mountains }\end{array}$ & Warts dry & $\begin{array}{l}\text { P. pondorosa } \\
\text { climax. } \\
\text { P. odulis } \\
\text { J. Geponono } \\
\text { J. monosporma }\end{array}$ & $\begin{array}{l}\text { P. adulle } \\
\text { P. arrobilormis } \\
\text { P. montogll(Co) } \\
\text { P. mrapata (CO) } \\
\text { J. doppoana }\end{array}$ & $\begin{array}{l}\text { F. arizonica } \\
\text { C. Iondfarl } \\
\text { o. gomballl } \\
\text { Rioos coroum } \\
\text { B. gracllls }\end{array}$ & 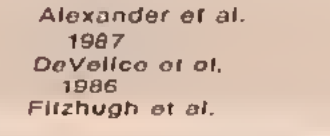 \\
\hline Denthonia perry/ phase (CO,NM) & $\begin{array}{l}\text { of southern } \\
\text { Colorado } \\
(9,500 \cdot 9,900)\end{array}$ & & $\begin{array}{l}\text { J. scopurorum } \\
\text { minor cllmaxes } \\
\text { In some phases } \\
\text { (AZ,NM) }\end{array}$ & $\begin{array}{l}\text { J. monosporme } \\
\text { J. scopulorum }\end{array}$ & $\begin{array}{l}\text { O. perryt } \\
\text { M. monrane } \\
\text { P. tendlertana } \\
\text { Haplopappus perryl }\end{array}$ & $\begin{array}{l}1987 \\
\text { Hanks el al. } \\
1983 \\
\text { Komarkova el al. } \\
1988 \\
\text { Radlofl } 1983\end{array}$ \\
\hline $\begin{array}{l}\text { Pinus ponderosel } \\
\text { Festuca ldehoens/s H.T. } \\
\text { F. ldahoensls (Iypla) phase } \\
\text { P. ponderosa phase (ID) } \\
\text { Arclostaphylos palule phase (UT) } \\
\text { Artemisle tridentela phase (UT) } \\
\text { Festuca scabrella phase (MT) }\end{array}$ & $\begin{array}{l}\text { Mountalns of } \\
\text { easlern Washingion, } \\
\text { idaho, central } \\
\text { and soulheaslern } \\
\text { Monlana, northern } \\
\text { Ulah, north- } \\
\text { cenlral Wyoming } \\
(2,500-6,000) \text {. } \\
\text { and south. } \\
\text { central Colorado } \\
(8,800.9,300)\end{array}$ & Warm dry & $\begin{array}{l}\text { P. pondarosa } \\
\text { cllmex }\end{array}$ & $\begin{array}{l}\text { Usually pure slands. } \\
\text { May conlain (UT) } \\
\text { Pinus contore } \\
\text { J. scopulorum } \\
\text { P. lremuloldes }\end{array}$ & $\begin{array}{l}\text { F. Idahoensls } \\
\text { A. patula } \\
\text { A. Inidentela } \\
\text { B. repens } \\
\text { P. tridentate } \\
\text { S. elbus } \\
\text { A. splcalum } \\
\text { Calamagrostis rubescens } \\
\text { F. scaberlla } \\
\text { Achlllee millelollum } \\
\text { B. sagilltata }\end{array}$ & $\begin{array}{l}\text { Cooper ef al. } 1987 \\
\text { Daubenmire and } \\
\text { Daubenmire } 1968 \\
\text { Hansen and } \\
\text { Holfman } 1988 \\
\text { Hoflmen and } \\
\text { Alexander } 1976 \\
\text { Komarkova ol al. } \\
\text { } 9888 \\
\text { Mauk and } \\
\text { Henderson } 1984 \\
\text { Pfisler et al. } 1977 \\
\text { Steele el al. } 1981\end{array}$ \\
\hline $\begin{array}{l}\text { Pinus ponderosel } \\
\text { Hesperochloa king/l H.T. }\end{array}$ & $\begin{array}{l}\text { Mounlalns of } \\
\text { north } \cdot \text { cenlral } \\
\text { Colorado } \\
(7,300 \cdot 8,400)\end{array}$ & Warm dry & $\begin{array}{l}\text { P. ponderosa } \\
\text { cllmax }\end{array}$ & $\begin{array}{l}\text { P. menzles" } \\
\text { P. \|exills }\end{array}$ & $\begin{array}{l}\text { H. kingll } \\
\text { R. cereum } \\
\text { K. crlstela } \\
\text { (K. mecrantha) } \\
\text { Allium geyerl } \\
\text { A. Irigida } \\
\text { G. Iremontil } \\
\text { Sedum slenopelelum }\end{array}$ & $\begin{array}{l}\text { Hess and } \\
\text { Alexander } 1986\end{array}$ \\
\hline $\begin{array}{l}\text { Pinus ponderosal } \\
\text { Muhlenbergla montena H.T. } \\
{[\text { P. ponderosa. }} \\
\text { Pseudotsuga menziesill } \\
\text { M. montena H.T.] } \\
{[\text { P. ponderosal }} \\
\text { Pos longlligule C.T.] }\end{array}$ & $\begin{array}{l}\text { Mountalns of } \\
\text { Arlzona and } \\
\text { New Mexico, } \\
\text { central and } \\
\text { soulhern Utah, } \\
\text { and central } \\
\text { and southern } \\
\text { Colorado } \\
(6,800.8,800)\end{array}$ & Warm ory & $\begin{array}{l}\text { P. ponderosa } \\
\text { cllmax. } \\
\text { P. edulis } \\
\text { J. deppeene } \\
\text { minor climaxes }\end{array}$ & $\begin{array}{l}\text { P. menzies/l } \\
\text { P. edulls } \\
\text { P. llexllis } \\
\text { J. deppoana } \\
\text { J. monosperma } \\
\text { J. scopulorum }\end{array}$ & $\begin{array}{l}\text { M. montana } \\
\text { B. repens } \\
\text { C. montanus } \\
\text { Q. gembelli } \\
\text { 8. graclils } \\
\text { P. lendlorlana } \\
\text { P. longligula } \\
\text { S. hystix } \\
\text { Senecio neomexicanus } \\
\text { Smllaclie stellata } \\
\text { Solldago canadensis } \\
\text { Yucce glauce }\end{array}$ & $\begin{array}{l}\text { Alexander et al. } \\
1987 \\
\text { DeVelice et al. } \\
1986 \\
\text { Fltzhugh el al. } \\
1987 \\
\text { Hanks ot al. } \\
1983 \\
\text { Hess and } \\
\text { Alexender } 1986 \\
\text { Muldavin el el. } \\
1986 \\
\text { Radloff } 1983 \\
\text { Youngblood ond } \\
\text { Mauk } 1985\end{array}$ \\
\hline $\begin{array}{l}\text { Pinus ponderosel } \\
\text { Muhlenbergla virescens H.T. } \\
\text { [P. pondorosa/M. virescens. } \\
\text { Fostuca arizonica H.T.] } \\
\text { [P. pondorosalM. virescens. } \\
\text { F. erizonicel } \\
\text { Bouteloua gracills C.T.] } \\
\text { M. virescens (lyplc) phase } \\
\text { Quorcus gambolli phase } \\
\text { B. gracllis phase (AZ) } \\
\text { F. arizonice phase }\end{array}$ & $\begin{array}{l}\text { Mountains of } \\
\text { Arizona and } \\
\text { soulhwestern } \\
\text { New Mexico } \\
(8,800 \cdot 9,300)\end{array}$ & Warm dry & $\begin{array}{l}\text { P. ponderosa } \\
\text { cllmax. } \\
\text { P. edulls } \\
\text { P. manziesil } \\
\text { P. strobllorm/s } \\
\text { Pinus engolmannll } \\
\text { J. deppeana } \\
\text { minor cllmaxes }\end{array}$ & $\begin{array}{l}\text { P. edulls } \\
\text { P. menzlesil } \\
\text { P. strobllormis } \\
\text { P. engelmannil } \\
\text { J. deppeana }\end{array}$ & $\begin{array}{l}\text { M. virescens } \\
\text { B. repens } \\
\text { C. lendierl } \\
\text { Quorcus spp. } \\
\text { B. gracllis } \\
\text { F. arizonice } \\
\text { M. montena } \\
\text { P. lendieriena } \\
\text { P. longlitigula } \\
\text { Cerex spp. } \\
\text { Soneclo wootonll }\end{array}$ & $\begin{array}{l}\text { Alexander el al. } \\
1987 \\
\text { DeVellce and } \\
\text { Ludwig } 1983 \\
\text { Fltzhugh ot al. } \\
1987 \\
\text { Henks el el. } \\
1983 \\
\text { Muldevin et al. } \\
1986\end{array}$ \\
\hline $\begin{array}{l}\text { Pinus ponderosal } \\
\text { Oryzopsis hymonoidos H.T. } \\
\text { (Sand hilie) }\end{array}$ & $\begin{array}{l}\text { Mountalns of } \\
\text { norlhern New } \\
\text { Mexico and } \\
\text { southern Colorado } \\
(5,500 \cdot 8,000)\end{array}$ & $\begin{array}{l}\text { Warm very } \\
\text { dry }\end{array}$ & $\begin{array}{l}\text { P. ponderosa } \\
\text { climax. } \\
\text { J. monosporma } \\
\text { minor cilmax }\end{array}$ & J. monosperma & $\begin{array}{l}\text { Q. hymenoldes } \\
\text { C. montanus } \\
\text { S. scoparium } \\
\text { S. hystrix } \\
\text { Heterothoca fulcreta }\end{array}$ & $\begin{array}{l}\text { DeVellce el al, } \\
\quad 1986\end{array}$ \\
\hline
\end{tabular}





\begin{tabular}{|c|c|c|c|c|c|c|}
\hline $\begin{array}{l}\text { Habltet lype or } \\
\text { community type }\end{array}$ & $\begin{array}{l}\text { Location end } \\
\text { alevation (foel) }\end{array}$ & Slto & $\begin{array}{l}\text { Successionel } \\
\text { status }\end{array}$ & $\begin{array}{c}\text { Troe } \\
\text { assocletes }\end{array}$ & $\begin{array}{l}\text { Princlpal undergrowth } \\
\text { spectes }\end{array}$ & Authorlity \\
\hline $\begin{array}{l}\text { Pinus pondarosal } \\
\text { Poa pratans/s H.T. } \\
\text { [Plnus ponderosal } \\
\text { Riparlan tores! H.T.] }\end{array}$ & $\begin{array}{l}\text { Mountains of } \\
\text { soulhwastern and } \\
\text { northern New } \\
\text { Mexlco end } \\
\text { southern Colorado } \\
(6.000 \cdot 8.500)\end{array}$ & Warm molsi & $\begin{array}{l}\text { P. pondorosa } \\
\text { climax }\end{array}$ & $\begin{array}{l}\text { Populus angus } 1 / 1 \text { olls } \\
\text { Acor nagundo }\end{array}$ & $\begin{array}{l}\text { P. pratansis } \\
\text { Ainus tonultollia } \\
\text { Q. gamballl } \\
\text { Gallum spp. } \\
\text { Ir/s missourionsis } \\
\text { Juncus spp. }\end{array}$ & $\begin{array}{l}\text { Alexander ol al. } \\
1987 \\
\text { DeVellce el al. } \\
1988\end{array}$ \\
\hline $\begin{array}{l}\text { Pinus pondarosal } \\
\text { Stipa comata H.T. }\end{array}$ & $\begin{array}{l}\text { Mountalns of } \\
\text { northern Idaho } \\
\text { and eastern } \\
\text { Weshington } \\
(2,500-3,000)\end{array}$ & $\begin{array}{l}\text { Warm very } \\
\text { dry }\end{array}$ & $\begin{array}{l}\text { P. pondarosa } \\
\text { climax }\end{array}$ & Usually pure slands & $\begin{array}{l}\text { S. comata } \\
\text { A. longisata } \\
\text { Poa sacunda } \\
\text { Stipa spp. }\end{array}$ & $\begin{array}{l}\text { Daubenmire and } \\
\text { Daubenmire } 1968\end{array}$ \\
\hline $\begin{array}{l}\text { Pinus ponderosal } \\
\text { Stipa occldentalls H.T. }\end{array}$ & $\begin{array}{l}\text { Mounteins of } \\
\text { centred Idaho } \\
(3,500 \cdot 4,800)\end{array}$ & $\begin{array}{l}\text { Warm very } \\
\text { dry }\end{array}$ & $\begin{array}{l}\text { P. ponderosa } \\
\text { climax }\end{array}$ & Usually pure slands & $\begin{array}{l}\text { S. occidantalls } \\
\text { P. Iridentala } \\
\text { Silpa thurbarlana }\end{array}$ & Sleole ot al. 1981 \\
\hline $\begin{array}{l}\text { Pinus pondarosal } \\
\text { Carax gayari H.T. }\end{array}$ & $\begin{array}{l}\text { Mountains of } \\
\text { norlhern Ulah } \\
(7,200-8,300) \text {. } \\
\text { and south. } \\
\text { eastern Wyoming } \\
(6,100-8,500)\end{array}$ & Cool dry & $\begin{array}{l}\text { P. pondarose } \\
\text { cllmax }\end{array}$ & $\begin{array}{l}\text { P. contorta (UT) } \\
\text { P. tremuloldos }\end{array}$ & $\begin{array}{l}\text { C. gayarl } \\
\text { B. repans } \\
\text { Pachisllma myrsinllas } \\
\text { Poa norvosa } \\
\text { A. cordllolla }\end{array}$ & $\begin{array}{l}\text { Alexander el al. } \\
1986 \\
\text { Mauk and } \\
\text { Henderson } 1984\end{array}$ \\
\hline $\begin{array}{l}\text { PInus ponderosal } \\
\text { Carex hellophylla H.T. }\end{array}$ & $\begin{array}{l}\text { Black Hills end } \\
\text { Bear Lodge } \\
\text { Mountains, South } \\
\text { Dakola and eastern } \\
\text { Wyoming }(4,500- \\
5,200) \text { : mounteins of } \\
\text { soulhwestern North } \\
\text { Dakote and soulh- } \\
\text { eastern Montana } \\
(3,900-4.000)\end{array}$ & $\begin{array}{l}\text { Warm very } \\
\text { dry }\end{array}$ & $\begin{array}{l}\text { P. pondarosa } \\
\text { cllmax }\end{array}$ & $\begin{array}{l}\text { Usually pure stands. } \\
\text { May conlain } \\
\text { J. scopulorum }\end{array}$ & $\begin{array}{l}\text { C. hallophylla } \\
\text { A. splcatum } \\
\text { Danthonla splcala } \\
\text { F. ldahoansis } \\
\text { P. pratensis } \\
\text { Silpa spp. } \\
\text { Astar clllolatus } \\
\text { Halerotheca villosa }\end{array}$ & $\begin{array}{l}\text { Hansen and } \\
\text { Hofiman } 1988 \\
\text { Hollman and } \\
\text { Alexander } 1987\end{array}$ \\
\hline $\begin{array}{l}\text { Inus ponderosa/ } \\
\text { Carex ross/I H.T. }\end{array}$ & $\begin{array}{l}\text { Front Range, } \\
\text { north-central } \\
\text { Colorado, and } \\
\text { mountatns of } \\
\text { southeaslern } \\
\text { Wyoming } \\
(5,800-6,400)\end{array}$ & $\begin{array}{l}\text { Warm dry } \\
10 \text { woll. } \\
\text { drained }\end{array}$ & $\begin{array}{l}\text { P. pondarosa } \\
\text { cllmax }\end{array}$ & $\begin{array}{l}\text { Usually pure slands. } \\
\text { May contaln } \\
\text { P. monzles// (CO) } \\
\text { J. scopulorum }\end{array}$ & $\begin{array}{l}\text { C. rossli } \\
\text { J. communis } \\
\text { K. cristata } \\
\text { (K. macrantha) } \\
\text { M. montana } \\
\text { A. lanulosa }\end{array}$ & $\begin{array}{l}\text { Alexander el al. } \\
1988 \\
\text { Hess and } \\
\text { Alexander } 1986\end{array}$ \\
\hline $\begin{array}{l}\text { Inus ponderosal } \\
\text { CInder Solls H.T. }\end{array}$ & $\begin{array}{l}\text { Mounlalns ol } \\
\text { north-ceniral } \\
\text { and norithwesiern } \\
\text { New Mexico } \\
(7,700 \cdot 8,500)\end{array}$ & Warm dry & $\begin{array}{l}\text { P. pondeross } \\
\text { cllmax. } \\
\text { P. edulls } \\
\text { minor cllmax }\end{array}$ & P. edulis & $\begin{array}{l}\text { Q. gambelll } \\
\text { A. cereum } \\
\text { B. gracllls } \\
\text { M. montana } \\
\text { Lupinus spp. }\end{array}$ & $\begin{array}{l}\text { Alexander el al. } \\
\quad 1987\end{array}$ \\
\hline $\begin{array}{l}\text { PInus ponderosal } \\
\text { Rockland H.T. }\end{array}$ & $\begin{array}{l}\text { Mountelns of } \\
\text { eastern Arlzona, } \\
\text { and northern and } \\
\text { soulhwestern New } \\
\text { Mexico } \\
(8.300-8.700)\end{array}$ & Warm dry & $\begin{array}{l}\text { P. pondarosa } \\
\text { cllmax. } \\
\text { P. monzlos/l } \\
\text { P. odulis } \\
\text { J. doppoana } \\
\text { minor cllmaxos } \\
\text { strobiformis a }\end{array}$ & $\begin{array}{l}\text { P. menzles/l } \\
\text { P. odulls } \\
\text { P. strobllorm/s } \\
\text { J. doppeana } \\
\text { oríos }\end{array}$ & $\begin{array}{l}\text { O. grisea } \\
\text { Boutoloua spp. } \\
\text { F. arlzonica } \\
\text { M. montana } \\
\text { M. virescens } \\
\text { Solldago spp. }\end{array}$ & $\begin{array}{l}\text { Alexander of el. } \\
1987 \\
\text { Flizhugh ef al. } \\
1987\end{array}$ \\
\hline $\begin{array}{l}\text { Pinus serobilormiaf } \\
\text { foutuoe Hixanical H.T. }\end{array}$ & $\begin{array}{l}\text { Mountaino of } \\
\text { northoin Afizonn } \\
\text { (7.500-0.000) }\end{array}$ & Wart ary & 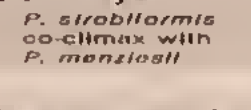 & $\begin{array}{l}\text { P. monzlos"l } \\
\text { P. ponderosu }\end{array}$ & $\begin{array}{l}\text { F. arizonica } \\
\text { B. ropons } \\
\text { Bromus onlufus } \\
\text { M. montana }\end{array}$ & $\begin{array}{l}\text { Motr and } \\
\text { Ludwid } 1979\end{array}$ \\
\hline \multicolumn{7}{|c|}{ Pseudotsuga menziesii series } \\
\hline $\begin{array}{l}\text { Pseudolsuga menzlesill } \\
\text { Acer glabrum H.T. } \\
\text { A. glabrum (lypic) phase } \\
\text { Pachistima myrsinlles phase } \\
\text { (ID,WY } \\
\text { Symphoricarpos oraophllus phase } \\
\text { (ID) }\end{array}$ & $\begin{array}{l}\text { Mountains of } \\
\text { contral and } \\
\text { soulheastern Idaho. } \\
\text { northwestern } \\
\text { Wyoming }(4,800 \\
8,300) \text {, and } \\
\text { northern Ulah } \\
(5,800 \cdot 7,700)\end{array}$ & Cool molst & $\begin{array}{l}\text { P. menzles/l } \\
\text { climax }\end{array}$ & $\begin{array}{l}\text { Ables grand/s } \\
\text { P. ponderosa } \\
\text { P. contorta } \\
\text { P. flexills } \\
\text { J. scopulorum } \\
\text { P. tremuloides }\end{array}$ & $\begin{array}{l}\text { A. glabrum } \\
\text { A. alnilolia } \\
\text { P. myrsinilas } \\
\text { P. virginiana } \\
\text { R. cereum } \\
\text { S. oreophllus } \\
\text { C. rubescens } \\
\text { A. cordllolla }\end{array}$ & $\begin{array}{l}\text { Mauk and } \\
\text { Henderson } 1984 \\
\text { Steele ol at. } \\
\text { 1981, } 1983\end{array}$ \\
\hline $\begin{array}{l}\text { Pseudolsuga menzlesil' } \\
\text { Acer grandrdenialum H.T. }\end{array}$ & $\begin{array}{l}\text { Mountains of } \\
\text { south.central } \\
\text { Arlzona } \\
(6,500 \cdot 7,000)\end{array}$ & Warm molst & $\begin{array}{l}\text { P. menzies ll } \\
\text { cllmax }\end{array}$ & $\begin{array}{l}\text { P. ponderosa } \\
\text { J. dappeana }\end{array}$ & $\begin{array}{l}\text { A. grandidentalum } \\
\text { Holodiscus dumosus } \\
\text { O. arizonica } \\
\text { Q. hypoleucoldas }\end{array}$ & $\begin{array}{l}\text { DeVelice and } \\
\text { Ludwlg } 1983\end{array}$ \\
\hline $\begin{array}{l}\text { Pseudolsuga menzlesill } \\
\text { Arcloslaphylos patula H.T. }\end{array}$ & $\begin{array}{l}\text { Mountalns of } \\
\text { cenlral and } \\
\text { southern Ulah } \\
(7,200 \cdot 8,700)\end{array}$ & Warm dry & $\begin{array}{l}\text { P. menzies/l } \\
\text { climax. } \\
\text { J. scopulorum } \\
\text { minor climax }\end{array}$ & $\begin{array}{l}\text { P. ponderosa } \\
\text { P. llexills } \\
\text { J. scopulorum }\end{array}$ & $\begin{array}{l}\text { A. patula } \\
\text { B. repens } \\
\text { Ceanothus martinil } \\
\text { S. oreophllus }\end{array}$ & $\begin{array}{l}\text { Youngblood and } \\
\text { Mauk } 1985\end{array}$ \\
\hline $\begin{array}{l}\text { Pseudolsuga manzlesill } \\
\text { Arclosiaphylos uva.ursi H.T. }\end{array}$ & $\begin{array}{l}\text { Mounlains of } \\
\text { central Montana } \\
(4,700-6,500) \\
\text { and soulh. } \\
\text { western New } \\
\text { Mexlco } \\
(9,500 \cdot 10,000)\end{array}$ & $\begin{array}{l}\text { Warm very } \\
\text { dry }\end{array}$ & $\begin{array}{l}\text { P. manzies/l } \\
\text { climax or } \\
\text { co-cilmax whith } \\
\text { P. strobltorm/s }\end{array}$ & $\begin{array}{l}\text { P. strobllorm/s (NM) } \\
\text { P. ponderosa } \\
\text { P. flexills (MT) } \\
\text { P. tremulo/des (NM) }\end{array}$ & $\begin{array}{l}\text { A. uva.ursl } \\
\text { A. spicalum } \\
\text { B. cllatus } \\
\text { Fesluca spp. } \\
\text { M. montana } \\
\text { B. sagitfata } \\
\text { Lithosparmum ruderale } \\
\text { Solldago spathulata }\end{array}$ & $\begin{array}{l}\text { Fitzhugh et al. } \\
\quad 1987 \\
\text { Pfister el at. } 1977\end{array}$ \\
\hline $\begin{array}{l}\text { Pseudolsuga manzlasill } \\
\text { Barberls rapans H.T. } \\
\text { B. rapans (Iyplc) phase } \\
\text { Pinus pondarosa phase (UT) } \\
\text { Junlparus communls phase } \\
\text { (ID,UT.WY) } \\
\text { Symphoricarpos oreophllus phase } \\
\text { (ID,UT,WY) } \\
\text { Carex geyarl phase (ID.UT) }\end{array}$ & $\begin{array}{l}\text { Mounlains of } \\
\text { central and } \\
\text { southeastern Idaho } \\
(4,500 \cdot 7,700) \text {, norlh. } \\
\text { western Wyoming } \\
(5,700 \cdot 8,500) \text {, and } \\
\text { norlhern Ulah }(5,400 \text {. } \\
9,700) \text { i mountalns } \\
\text { of north central } \\
\text { Wyoming }(7,000 \\
8,500) \text {, and } \\
\text { western Colorado } \\
(8,000 \cdot 9,900)\end{array}$ & $\begin{array}{l}\text { Warm dry } \\
\text { to well- } \\
\text { dralned }\end{array}$ & $\begin{array}{l}\text { P. manzles/l } \\
\text { climax. } \\
\text { P. pondarosa } \\
\text { P. contorta } \\
\text { P. llaxllis } \\
\text { minor cllmaxes }\end{array}$ & $\begin{array}{l}\text { P. pondarosa } \\
\text { P. contorta } \\
\text { P. Haxllis } \\
\text { A. grand/s (Nol WY) } \\
\text { J. scopulorum } \\
\text { P. Iremuloides }\end{array}$ & $\begin{array}{l}\text { B. repens } \\
\text { J. communis } \\
\text { P. myrsinites } \\
\text { S. oreophlius } \\
\text { C. geyerl } \\
\text { C. rossill } \\
\text { A. cordilolla } \\
\text { Gallum saptentrionala } \\
\text { Smilacina racamosa }\end{array}$ & $\begin{array}{l}\text { Hoflman } 1988 \\
\text { Hoflman ond } \\
\text { Alexander } 1976 \\
\text { Mauk and } \\
\text { Henderson } 1984 \\
\text { Sleele ot al. } \\
\text { 1981. 1983 } \\
\text { Youngblood and } \\
\text { Mauk 1985 }\end{array}$ \\
\hline $\begin{array}{l}\text { Psaudotsuga menzlasill } \\
\text { Carcocarpus ledilollus H.T. }\end{array}$ & $\begin{array}{l}\text { Mountains of } \\
\text { southeaslern and } \\
\text { central Idaho, } \\
\text { and Ufah } \\
(8,000 \cdot 8,100)\end{array}$ & Warm dry & $\begin{array}{l}\text { P. menzies/l } \\
\text { climax or } \\
\text { co. cllmax with } \\
\text { P. pondarosa. } \\
\text { P. llexlls } \\
\text { J. scopulorum } \\
\text { minor cllmaxes }\end{array}$ & $\begin{array}{l}\text { P. ponderosa } \\
\text { P. llexills } \\
\text { J. scopulorum } \\
\text { P. Iramuloides }\end{array}$ & $\begin{array}{l}\text { C. ladilollus } \\
\text { B. repans } \\
\text { S. oraophllus } \\
\text { A. spicatum } \\
\text { A. cordllolla } \\
\text { B. saglitata } \\
\text { Crapls aluminata }\end{array}$ & $\begin{array}{l}\text { Mauk and } \\
\text { Henderson } 1984 \\
\text { Sleele el al. } \\
1981,1983 \\
\text { Youngblood and } \\
\text { Mauk 1985 }\end{array}$ \\
\hline $\begin{array}{l}\text { Psaudolsuga manzlasill } \\
\text { Carcocarpus montanus H.T. }\end{array}$ & $\begin{array}{l}\text { Mountalns of } \\
\text { centrel and } \\
\text { soulhern Utah } \\
(7,200-8,200)\end{array}$ & Warm dry & $\begin{array}{l}\text { P. manzlasil } \\
\text { climax. } \\
\text { P. adulls } \\
\text { J. ostoospermo } \\
\text { J. scopulorum } \\
\text { minor cllmaxes }\end{array}$ & $\begin{array}{l}\text { P. adulls } \\
\text { J. osteosparma } \\
\text { J. scopulorum }\end{array}$ & $\begin{array}{l}\text { C. montanus } \\
\text { B. repans } \\
\text { J. communis } \\
\text { Shepherdla rotundilolla } \\
\text { S. oreophllus }\end{array}$ & $\begin{array}{l}\text { Youngblood and } \\
\text { Mauk } 1985\end{array}$ \\
\hline $\begin{array}{l}\text { Pseudolsuga manziasilit } \\
\text { Clamalis psaudoalpina H.T. }\end{array}$ & $\begin{array}{l}\text { Front Range, } \\
\text { central Colorado } \\
(7,800 \cdot 9,300)\end{array}$ & $\begin{array}{l}\text { Warm well. } \\
\text { dralned }\end{array}$ & $\begin{array}{l}\text { P. monzlesil } \\
\text { climax }\end{array}$ & $\begin{array}{l}\text { P. ponderosa } \\
\text { P. llaxllis } \\
\text { P. tremuloldas }\end{array}$ & $\begin{array}{l}\text { C. pseudoalpina } \\
\text { J. communis } \\
\text { Rosa spp. } \\
\text { Calamagrostis purpurascans } \\
\text { Carex spp. } \\
\text { Fragarla spp. } \\
\text { Saxilraga bronchlalls } \\
\text { Thallctrum fandlarl } \\
\text { Valerlana adulls }\end{array}$ & $\begin{array}{l}\text { Radlofl } 1983 \\
\text { s }\end{array}$ \\
\hline
\end{tabular}





\begin{tabular}{|c|c|c|c|c|c|c|}
\hline $\begin{array}{l}\text { Hebltat type or } \\
\text { community type }\end{array}$ & $\begin{array}{l}\text { Locetion end } \\
\text { elevetion (foet) }\end{array}$ & Slte & $\begin{array}{l}\text { Successlonal } \\
\text { etetue }\end{array}$ & $\begin{array}{c}\text { Tree } \\
\text { essocletee }\end{array}$ & $\begin{array}{l}\text { Princlpal undergrowth } \\
\text { spectes }\end{array}$ & Authority \\
\hline $\begin{array}{l}\text { Pseudolsuga menzles III } \\
\text { Holod/scus dumosus H.T. } \\
\text { (Scree lorest) }\end{array}$ & $\begin{array}{l}\text { Mountains of } \\
\text { norlhern and } \\
\text { southwestern } \\
\text { New Mexico, and } \\
\text { southern Colorado } \\
(9,600 \cdot 9,900)\end{array}$ & Warm dry & $\begin{array}{l}\text { P. menziesil } \\
\text { cllmax or } \\
\text { co.cllmax with } \\
\text { P. strobllormis }\end{array}$ & $\begin{array}{l}\text { P. strobllormis } \\
\text { Ables laslocarpa } \\
\text { Plcea engolmennll } \\
\text { P. Hexills } \\
\text { P. tremuloldes }\end{array}$ & $\begin{array}{l}\text { H. dumosus } \\
\text { C. montanus } \\
\text { Jamesta americena } \\
\text { Ribes spp. } \\
\text { Sall scouleriana } \\
\text { S. oreophllus }\end{array}$ & $\begin{array}{l}\text { Devefice ot al. } \\
1988 \\
\text { Flizhugh ot al. } \\
1987\end{array}$ \\
\hline $\begin{array}{l}\text { Pseudolsuga menzlesil' } \\
\text { Jamesla americane H.T. }\end{array}$ & $\begin{array}{l}\text { Front Range, } \\
\text { norlh-centrai } \\
\text { Colorado, and } \\
\text { mountalns of } \\
\text { south.central } \\
\text { Colorado } \\
(7,200 \cdot 9,800)\end{array}$ & $\begin{array}{l}\text { Cool dry } \\
\text { lo well. } \\
\text { drained }\end{array}$ & $\begin{array}{l}\text { P. menziesil } \\
\text { cllmax }\end{array}$ & $\begin{array}{l}\text { P. ponderose } \\
\text { P. contorta } \\
\text { P. llexilis } \\
\text { J. scopulorum }\end{array}$ & $\begin{array}{l}\text { J. americana } \\
\text { A. glabrum } \\
\text { J. communis } \\
\text { P. monogynus } \\
\text { Fragarla ovalls } \\
\text { (F. virginiana) } \\
\text { Polentlila llssa }\end{array}$ & $\begin{array}{l}\text { Hess end } \\
\text { Alexander } 1986 \\
\text { Komarkova el al. } \\
1988 \\
\text { Radlofl } 1983\end{array}$ \\
\hline $\begin{array}{l}\text { Pseudolsuge menzlesili' } \\
\text { Juniperus communis H.T. }\end{array}$ & $\begin{array}{l}\text { Mountalns of } \\
\text { cenlral and } \\
\text { southweslern } \\
\text { Montana }(6,400 \text {. } \\
\text { 7,800), ceniral } \\
\text { Ideho, and north. } \\
\text { western Wyoming } \\
(7,400 \cdot 10,300)\end{array}$ & $\begin{array}{l}\text { Cool dry } \\
\text { to exces. } \\
\text { slvely } \\
\text { drained }\end{array}$ & $\begin{array}{l}\text { P. menziesil } \\
\text { cllmax }\end{array}$ & $\begin{array}{l}\text { P. contorto } \\
\text { P. llexlils } \\
\text { J. scopulorum }\end{array}$ & $\begin{array}{l}\text { J. communis } \\
\text { juniperus horizontalis } \\
\text { S. canadensis } \\
\text { S. oreophllus } \\
\text { A. cordllolle } \\
\text { A. miser } \\
\text { F. ovalis } \\
\quad \text { (F. virginana) }\end{array}$ & $\begin{array}{l}\text { Plister el al. } \\
1977 \\
\text { Steele el al. } \\
1981,1983\end{array}$ \\
\hline $\begin{array}{l}\text { PSeudolsuga menziesill } \\
\text { Linnaea borealls H.T. } \\
\text { L. borealls (typic) phose } \\
\text { Symphoricarpos albus phase (MT) } \\
\text { Vaccinlum globulare phase (MT) } \\
\text { Calamagrostis rubescens phase } \\
\text { (MT) }\end{array}$ & $\begin{array}{l}\text { Mountains of } \\
\text { central and } \\
\text { northwestern } \\
\text { Montana, and } \\
\text { cenlral Ideho } \\
(2,600-6,500)\end{array}$ & $\begin{array}{l}\text { Warm molst } \\
\text { to well. } \\
\text { dralned }\end{array}$ & $\begin{array}{l}\text { P. menziosll } \\
\text { cllmax }\end{array}$ & $\begin{array}{l}\text { P. ponderosa } \\
\text { P. contorta } \\
\text { Larly occldentalls }\end{array}$ & $\begin{array}{l}\text { L. borealls } \\
\text { S. albus } \\
\text { S. betullollo } \\
\text { V. globulare } \\
\text { C. rubescens } \\
\text { A. cordllolla }\end{array}$ & $\begin{array}{l}\text { Pflster et al. } \\
1977 \text {. } \\
\text { Steele et al. } \\
1981\end{array}$ \\
\hline $\begin{array}{l}\text { Pseudolsuga menzlesill } \\
\text { Pachistima myrsiniles H.T. } \\
\text { P. menzlesillP. myrsiniles. } \\
\text { Carex geyerl H.T.] }\end{array}$ & $\begin{array}{l}\text { Mountains of } \\
\text { centrat and } \\
\text { western Coloredo } \\
(7,100-10,000)\end{array}$ & $\begin{array}{l}\text { Cool dry } \\
10 \text { well. } \\
\text { drained }\end{array}$ & $\begin{array}{l}\text { P. menzlesll } \\
\text { climex }\end{array}$ & $\begin{array}{l}\text { Picca engelmannll } \\
\text { P. comtorta } \\
\text { P. tromuloldes }\end{array}$ & $\begin{array}{l}\text { P. myrsinites } \\
\text { B. repens } \\
\text { Q. gambelli } \\
\text { S. oreophilus } \\
\text { Vaccinlum myrlillus } \\
\text { C. geyerl } \\
\text { A. cordilolla }\end{array}$ & $\begin{array}{l}\text { Hess and Wasser } \\
1982 \\
\text { Holfman and } \\
\text { Alexander } 1980 . \\
1983 \\
\text { Komarkova et al. } \\
1988\end{array}$ \\
\hline $\begin{array}{l}\text { Pseudolsuga menzlesill } \\
\text { Physocarpus malvaceus H.T. } \\
\text { P. malvaceus (Iyplc) phose } \\
\text { Pseudolsuga menzlesil phose } \\
\text { Pinus ponderosa phase (ID) } \\
\text { Pachislima myrsinites phase } \\
\text { (ID,Wn } \\
\text { Calamagrosils rubescons phase } \\
\text { (ID,MT) } \\
\text { Smllacina stellata phase (ID) }\end{array}$ & $\begin{array}{l}\text { Mountoins of } \\
\text { eastern Washinglon, } \\
\text { Ideho, Montane } \\
(2,000 \cdot 7,100) \\
\text { northwestern } \\
\text { Wyoming }(5,400 . \\
7,500) \text {, and Ulah } \\
(5,000 \cdot 9,100)\end{array}$ & $\begin{array}{l}\text { Cool molst } \\
\text { to well. } \\
\text { drained }\end{array}$ & $\begin{array}{l}\text { P. menzles } \\
\text { climex }\end{array}$ & $\begin{array}{l}\text { P. ponderosa } \\
\text { P. contorta } \\
\text { P. flexills } \\
\text { L. occldentalls } \\
\text { J. scopulorum } \\
\text { P. tramuloldes }\end{array}$ & $\begin{array}{l}\text { P. malvaceus } \\
\text { A. alnilolla } \\
\text { B. repens } \\
\text { H. discolor } \\
\text { P. myrsinites } \\
\text { S. albus } \\
\text { C. rubescens } \\
\text { C. geyerl } \\
\text { A. cordllolla } \\
\text { S. stellata }\end{array}$ & $\begin{array}{l}\text { Cooper el al. } 1987 \\
\text { Daubenmire and } \\
\text { Daubenmire } 1968 \\
\text { Mauk and } \\
\text { Henderson } 1984 \\
\text { Plister ot al. } 1977 \\
\text { Sieele el al. } \\
\text { 1981, } 1983 \\
\text { Youngblood and } \\
\text { Mauk 1985 }\end{array}$ \\
\hline $\begin{array}{l}\text { Pseudorsuga menzies } I I \\
\text { Physocerpus monogynus H.T. }\end{array}$ & 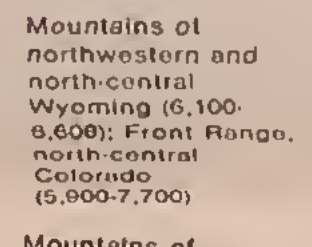 & $\begin{array}{l}\text { Warm well. } \\
\text { dratned }\end{array}$ & $\begin{array}{l}\text { P. menziesil } \\
\text { climax }\end{array}$ & $\begin{array}{l}\text { P. ponderosa } \\
\text { P. contorra } \\
\text { P. Hox } 1 / 1 / 5 \\
\text { J. scopulorum }\end{array}$ & $\begin{array}{l}\text { P. monogynus } \\
\text { B. repens } \\
\text { J. americana } \\
\text { S. borullilla } \\
\text { S. oreophlifus } \\
\text { H. Kingll } \\
\text { P. pratons/s } \\
\text { Q. Iromontll }\end{array}$ & $\begin{array}{l}\text { Hess and } \\
\text { Alexander } 1986 \\
\text { Hollman and } \\
\text { Alexander } 1976 \\
\text { Sreele el al. } 1983\end{array}$ \\
\hline $\begin{array}{l}\text { Pseudotsuga menziosil' } \\
\text { Purshia tridentata H.T. } \\
{[\text { [P. menzlesill }} \\
\text { Arclostaphylos uva. ursi H.T.] } \\
\text { Pinus ponderosal } \\
\text { Arlemisla inldeniala H.T.] }\end{array}$ & $\begin{array}{l}\text { Mountatns of } \\
\text { south.central } \\
\text { Colorado } \\
(8,800-9,800)\end{array}$ & Warm dry & $\begin{array}{l}\text { P. menzles } \\
\text { cllmax }\end{array}$ & $\begin{array}{l}\text { P. ponderosa } \\
\text { P. contorta } \\
\text { P. tremuloldes }\end{array}$ & $\begin{array}{l}\text { P. Iridentata } \\
\text { A. Iridenrata } \\
\text { A. Uva-urst } \\
\text { J. communis } \\
\text { K. cristata } \\
\text { (K. mocrantha) } \\
\text { C. loonea }\end{array}$ & $\begin{array}{l}\text { Komarkova at al. } \\
1988\end{array}$ \\
\hline $\begin{array}{l}\text { Pseudolsuga menzlesill' } \\
\text { Ouercus arizonica H.T. }\end{array}$ & $\begin{array}{l}\text { Mounlains ol } \\
\text { soulh.central } \\
\text { Arlzona } \\
(5,800-7,000)\end{array}$ & $\begin{array}{l}\text { Warm very } \\
\text { dry }\end{array}$ & $\begin{array}{l}\text { P. menzlesh } \\
\text { cllmax or } \\
\text { co-cllmax wilh } \\
\text { P. ponderosa }\end{array}$ & $\begin{array}{l}\text { P. ponderosa } \\
\text { P. dlscolor } \\
\text { J. deppeana }\end{array}$ & $\begin{array}{l}\text { Q. arizonica } \\
\text { a. gambelli } \\
\text { Q. hypoleucoldes } \\
\text { M. longliliguia }\end{array}$ & $\begin{array}{l}\text { Dovelice and } \\
\text { Ludwig } 1983 \\
\text { Muldavin ot al. } \\
1986\end{array}$ \\
\hline $\begin{array}{l}\text { Pseudotsuga menziesill } \\
\text { Ouercus gambelll H.T. } \\
\text { Q. gambelli (typlc) phase } \\
\text { Holodiscus dumosus phase (NM) } \\
\text { Fesiuca arizonlca phase (NM) } \\
\text { Muhlenbergla virescens phase } \\
\text { (AZ,NM) }\end{array}$ & $\begin{array}{l}\text { Mounialns ol } \\
\text { Now Mexico, } \\
\text { Arlzone, } \\
\text { southern Utah, } \\
\text { and southern } \\
\text { Colorado } \\
(6,500 \cdot 9,600)\end{array}$ & Warm dry & $\begin{array}{l}\text { P. menzlesll } \\
\text { climex or } \\
\text { co.cllmax wilh } \\
\text { P. ponderosa } \\
\text { P. strobllormis } \\
\text { J. scopulorum } \\
\text { minor climaxes }\end{array}$ & $\begin{array}{l}\text { P. ponderosa } \\
\text { P. strobllormis } \\
\text { P. edulls } \\
\text { Pinus engelmannll } \\
\text { J. deppeana } \\
\text { J. scopulorum }\end{array}$ & $\begin{array}{l}\text { Q. gambelli } \\
\text { H. dumosus } \\
\text { P. myrsinites } \\
\text { S. oreophllus } \\
\text { F. arizonica } \\
\text { M. montana } \\
\text { M. virescens } \\
\text { P. fondieriana } \\
\text { C. rossil }\end{array}$ & $\begin{array}{l}\text { Alexander et al. } \\
1984 \theta, 1984 \mathrm{~b} . \\
1987 \\
\text { DeVellice ol al. } \\
1986 \\
\text { DeVellce and } \\
\text { Ludwig } 1983 \\
\text { Filzhugh el al. } \\
\text { 1987 } \\
\text { Muldavin ol al. } \\
1986 \\
\text { Youngblood and } \\
\text { Mauk 1985 }\end{array}$ \\
\hline $\begin{array}{l}\text { Seudotsuga menziesill } \\
\text { Quercus hypoleucoldes H.T. }\end{array}$ & $\begin{array}{l}\text { Mountalns of } \\
\text { soulh central and } \\
\text { eestern Arizona, } \\
\text { and southwestern } \\
\text { New Mexlco } \\
(6,800-8,800)\end{array}$ & $\begin{array}{l}\text { Warm dry } \\
\text { to well. } \\
\text { dralned }\end{array}$ & $\begin{array}{l}\text { P. menzlosil } \\
\text { cllmax or } \\
\text { co.cllmax with } \\
\text { P. ponderosa }\end{array}$ & $\begin{array}{l}\text { P. ponderosa } \\
\text { P. strobllorm/s } \\
\text { Pinus engelmannil } \\
\text { P. discolor } \\
\text { P. edulls } \\
\text { Ables concolor }\end{array}$ & $\begin{array}{l}\text { Q. hypoleucoldes } \\
\text { Agave spp. } \\
\text { Q. arizonica } \\
\text { Q. gambelli } \\
\text { o. rugosa } \\
\text { Qpuntia spp. } \\
\text { M. longligula } \\
\text { P. fendieriana }\end{array}$ & $\begin{array}{l}\text { DeVellce and } \\
\text { Ludwwlg } 1983 \\
\text { Fllzhugh el al. } \\
1987 \\
\text { Molr and } \\
\text { Ludwig } 1979 \\
\text { Muldevin et al. } \\
\text { 1986 }\end{array}$ \\
\hline $\begin{array}{l}\text { PSeudolsuga menziesill } \\
\text { Quercus rugosa H.T. }\end{array}$ & $\begin{array}{l}\text { Mountalns of } \\
\text { soulh.ceniral } \\
\text { Arlzona } \\
(8,500-8,700)\end{array}$ & $\begin{array}{l}\text { Warm to } \\
\text { well. } \\
\text { drained }\end{array}$ & $\begin{array}{l}\text { P. menziesil } \\
\text { cllmax }\end{array}$ & $\begin{array}{l}\text { P. ponderosa } \\
\text { P. strobilorm/s }\end{array}$ & $\begin{array}{l}\text { Q. rugoso } \\
\text { a. hypoloucoldes }\end{array}$ & $\begin{array}{l}\text { DeVelice and } \\
\text { Ludw/g } 1983 \\
\text { Muldavin et el. } \\
1986\end{array}$ \\
\hline $\begin{array}{l}\text { Pseudotsuga menzlesill } \\
\text { Splraea betulliolla H.T. } \\
\text { S. betullolla (typlc) phese } \\
\text { Pinus ponderosa phase (ID) } \\
\text { Calamagrostis rubescens phase } \\
\text { (ID,WY) }\end{array}$ & $\begin{array}{l}\text { Mountalns of } \\
\text { central Montana, } \\
\text { Idaho }(3,300-8,100) \text {, } \\
\text { and northwestern } \\
\text { Wyoming } \\
(6,000 \cdot 8,200)\end{array}$ & Worm dry & $\begin{array}{l}\text { P. menzles"l } \\
\text { cllmex }\end{array}$ & $\begin{array}{l}\text { P. ponderosa } \\
\text { P. contorla } \\
\text { P. flexills }\end{array}$ & $\begin{array}{l}\text { S. belulliolla } \\
\text { A. ainilolla } \\
\text { B. repans } \\
\text { C. rubescens } \\
\text { A. corallolia } \\
\text { F. ovells } \\
\text { (F. virginiana) }\end{array}$ & $\begin{array}{l}\text { Cooper et al. } 1987 \\
\text { Pfister et al. } 1977 \\
\text { Steele et al. } \\
\text { 1981, } 1983\end{array}$ \\
\hline $\begin{array}{l}\text { Psoudolsuga menzlesill } \\
\text { Symphoricarpos albus H.T. } \\
\text { S. albus (Iyplc) phase } \\
\text { Pinus ponderosa phase (ID) } \\
\text { agropyron spicalum phase (MT) } \\
\text { Calamagrosils rubescens phaso } \\
\text { (ID,MT) }\end{array}$ & $\begin{array}{l}\text { Mountelns of } \\
\text { easlern Washinglon, } \\
\text { Idaho, Montana } \\
(2,700 \cdot 7,200) \text {. } \\
\text { and norihweslern } \\
\text { Wyoming } \\
(5,700 \cdot 7,400)\end{array}$ & Warm dry & $\begin{array}{l}\text { P. menzies } \\
\text { climex }\end{array}$ & $\begin{array}{l}\text { P. ponderosa } \\
\text { P. contorta } \\
\text { L. occidentalls } \\
\text { P. tromuloides }\end{array}$ & $\begin{array}{l}\text { S. albus } \\
\text { P. virginiana } \\
\text { Rosa spp. } \\
\text { S. betulliolia } \\
\text { A. spicalum } \\
\text { C. rubescens } \\
\text { Fostuca spp. } \\
\text { C. geyorl }\end{array}$ & $\begin{array}{l}\text { Cooper el el, } 1987 \\
\text { Deubenmlre and } \\
\text { Daubenmire } 1968 \\
\text { Plister el al. } 1977 \\
\text { Steele et al. } \\
\text { 1981, } 1983\end{array}$ \\
\hline $\begin{array}{l}\text { Soudotsuga monzlesill' } \\
\text { Symphoricarpos oreophlius H.T. }\end{array}$ & $\begin{array}{l}\text { Mounlains of } \\
\text { contral Idaho, } \\
\text { southweslern } \\
\text { Monlena, north. } \\
\text { western Wyoming } \\
(4,500.8,300) \text {. } \\
\text { norlhern and } \\
\text { soulhern Ulah, } \\
\text { and conlral and } \\
\text { soulhern Colorado } \\
(7,000.9800)\end{array}$ & Warm dry & $\begin{array}{l}\text { P. menziesil } \\
\text { cilmex. } \\
\text { P. ponderosa } \\
\text { P. flexills } \\
\text { J. scopulorum } \\
\text { minor climaxes }\end{array}$ & $\begin{array}{l}\text { P. ponderosa } \\
\text { P. contorta } \\
\text { P. floxills } \\
\text { J. scopulorum } \\
\text { P. tromuloldes }\end{array}$ & $\begin{array}{l}\text { C. geyerl } \\
\text { S. oreophlius } \\
\text { A. Iridentata } \\
\text { B. repens } \\
\text { P. virginiana } \\
\text { Aibes spp. } \\
\text { H. kingll } \\
\text { C. gevorl } \\
\text { Siollaria jamesiena } \\
\text { r. fondiol }\end{array}$ & $\begin{array}{l}\text { Hess and Wasser } 1982 \\
\text { Komerkova et al. } \\
1988 \\
\text { Mauk and } \\
\text { Honderson } 1984 \\
\text { Plister ol al. } 1977 \\
\text { Sleele el el. } \\
1981,1983 \\
\text { Youngblood ond } \\
\text { and Mouk 1985 }\end{array}$ \\
\hline
\end{tabular}





\section{Heblet typo or

Hebltet type or
communlty type \\ Pseudotsuga menzlesill \\ Vaccinium caespilosum H.T. \\ Pseudolsuga menzlesill Vaccinlum globulare H.T. V. globulare (typlc) phase
Arctostap hylos uye.ursl phase (M) Xerophyllum tenax phase (MT) Pseudolsuga menzles III}

centrel tdaho,

and west-centrat

Montena Agropyron spicalum H.T.

Mountetns of
north.central
Montane, and
Idaho
$(4,300 \cdot 7,400)$

Cool dry

$(4.300 \cdot 7,400)$

Mountalns ol end northern and centret ldeho
$(3.800 \cdot 7,500)$ Pseudolsuga menzies"ll
Bromus clllatus H.T.

Mountalns of Mountains of
southwestern New Mexico $(9,000 \cdot 10,000)$

Pseudotsuga menzlesilI Catamagrostis rubascens H.T. C. rubescens (Iyptc) phas P. Thus ponderosa phese (ID,MT) Arciosiaphyid
(ID,MN

Pachistima myrsintles phese (ID,Wr)

agropyron splcasum phase (M) Festuca idahoensis phese (ID)

$\rho_{\text {seudolsuga menziesilI }}$ Festuca arizonica H.T.

Mountains of eastern Washington. tdeho, Montena, $(4,100 \cdot 7,900)$, and northwestern Wyoming $(6,000-8,100)$

Mounteins ol northern end southwestern New Mexlco, eeste southern Colorado $(8,200-10,000)$

Pseudolsuga menzlasil'
Festuca idahoensis H.T.
F. Idahoens/s (typlc) phese F. Idahoens/s (lyplc) phese
Pinus ponderosa phasa (ID)

Mountalns of

Montane, northern

and centsal tdeho

$(3,000-8,000)$

and soulh-centra
Colorado

$(\theta, 000 \cdot 10.000)$

\begin{tabular}{|c|c|}
\hline $\begin{array}{l}\text { Pseudolsuga menzles'll } \\
\text { Festuca scabrella H.T. }\end{array}$ & $\begin{array}{l}\text { Mountelns of } \\
\text { central and } \\
\text { northwestern } \\
\text { Montana } \\
(2,700-7,400)\end{array}$ \\
\hline $\begin{array}{l}\text { Pseudolsuga menzlesil' } \\
\quad \text { Muhlenbergia montana H.T. }\end{array}$ & $\begin{array}{l}\text { Mountalns of } \\
\text { southwestern } \\
\text { New Mexico and } \\
\text { eastern Arlzona } \\
(7,500-9,800)\end{array}$ \\
\hline $\begin{array}{l}\rho_{\text {seudolsuga menzlesill }} \\
\text { Muhlenbargla virescens H.T. } \\
\text { [p. menzlesll. } \\
\text { Pinus strobllormisl } \\
\text { M. virascens H.T.] }\end{array}$ & $\begin{array}{l}\text { Mountains ol } \\
\text { southwestern } \\
\text { New Mexlco and } \\
\text { Aflzona } \\
(7,800-9,400)\end{array}$ \\
\hline
\end{tabular}

$\rho_{\text {seudolsuga menzlesil }}$ Carex gayeri H.T.

C. geyeri (typlc) phase

Mountalns ol

Montana east of

Continental

Divlde $(6,100$.

Symphoricarpos oreophilus phase 7,600 ) and northern

and central Idaho

$(3,700 \cdot 8,700)$;

Front Range and

mountains of

south-central

$(7,800 \cdot 8,800)$

Psaudolsuga menzlesill

Carex rossil H.T.

Front Range o

north.central

Colorado

Mounteins of

central and

southwestern

Montana, centrel

end southeestern
Idaho $(5,900 \cdot 8,600)$,

end northwestern

Wyoming $(6,900 \cdot 9,500)$

$\rho_{\text {seudotsuga menzlasil }}$

Osmorhiza chillensis H.T.

Mountelns of

central and

southeeslern

Ideho, and

$(5,300-7,800)$

Mounteins of

north-centrot

Pseudolsuga menziasil

Sparse H.T.
p. manziesill

Barbaris repens H.T.

Warm dry

Cool dry

\begin{tabular}{|c|c|}
\hline Site & $\begin{array}{l}\text { Succesetonet } \\
\text { etetue }\end{array}$ \\
\hline$m$ motst & $\begin{array}{l}\text { P. menzies } / \\
\text { climex }\end{array}$ \\
\hline ol dry & $\begin{array}{l}\text { P. menzlosil } \\
\text { climex }\end{array}$ \\
\hline in very & $\begin{array}{l}\text { P. menzlesil } \\
\text { co-cllmax with } \\
\text { P. ponderosa. } \\
\text { p. llexllis } \\
\text { J. scopuiorum } \\
\text { minor cllmaxes }\end{array}$ \\
\hline $\begin{array}{l}\text { of motst } \\
\text { wet }\end{array}$ & $\begin{array}{l}\text { P. menzles/l } \\
\text { climax. } \\
\text { P. ponderosa } \\
\text { P. strobllormis } \\
\text { minor cllmaxes }\end{array}$ \\
\hline $\begin{array}{l}\text { ol dry } \\
\text { wetl. } \\
\text { ined }\end{array}$ & $\begin{array}{l}\text { P. menzlesil } \\
\text { climax }\end{array}$ \\
\hline
\end{tabular}

$\begin{gathered}\text { Trae } \\ \text { eeeocletee }\end{gathered}$
conderosa
contorta
occidentalls

Princtpal undergrowth epecles

V. crespllosum

A. uve.ursi

L. borealls
S. albus

S. albus
C. rubescens

C. geyeri

V. giobulare

A. uva-ursl

C. geyerl
A. cordllolle

Osmorhiza chilensis

$x$. tenax

A. spicatum

F. idahoensis

B. sogiltata
M. bulbosa

scopulorum

P. ponderosa

Iremuloldes

B. clllalus

P. fendierlana

Erigaron eximius
(E. suparbus)

P. ponderosa

P. contort

L. occidentalis

C. rubescens

A. uva.ursl
B. repens

B. repens
P. myrsinites

A. spicalum

F. Idahoens/s

C. geyerl

A. cord/lolla

Smilacina amplexiceulis

Werm dry P. menzlesll

climex

P. ponderosa

P. strobllorm

P. Ilaxills

Plnus aristata
P. edulls

j. edoris

J. scopulorum

p. Iramuloldas

Werm dry

P. menzlesil

P. ponderosa

cllmax or
co.ctimex with

P. ponderosa

F. arizonica

A. uve ursi

H. dumosus

O. gambell

K. cristata

(K. macrantha)

M. montana

F. Idahoensis

A. alnllolla

P. virginian

Rosa spp.

A. splcatum
C. rubescens

Erlogonum spp.

Warm dry

P. menzlesi

co.ctlmax with

P. ponderos

minor cllmax

Warm dry

P. menziesil

climax or

co.climax with

P. pondarosa

P. strobllormis

P. edulls

J. deppeans

J. scopulorum

P. menzles/l

P. ponderosa

cllmax or P. stroblformis

co-climex with A. concolor

p. strobllorm/s. P. tremuloides

A. concolor

minor cllmax

P. menzles"I

P. ponderosa

P. contorla

L. occldentalis

P. Iremuloldes (CO)

F. scabrella

F. Idahoensis

A. sp/calum
K. cristala

(K. macrantha)

M. montana

B. rapans

O. gambell

P. Tandienlana

Girnium caespllosum

M. virescens

C. Iendierl

O. gambelli

B. clliatus

C. rossil

Garantum richardsonil

F. ovalis

(F. virginiana)

C. geyerl

A. uva-ursl

S. oreophllus

A. splcalum

A. spicalum

A. lanulosa

Fragaria spo.

Cooper of al. 1987

Pllster et at. 1977

1981,1983

Cooper et al. 1987 Steele ot al. 1981

Atexander et al.

1987

Fttzhugh of al.

1987

Cooper et al. 1987

Cooper et al. 1987

Daubenmire 1968

Mauk and 1984

Pilster et al. 1977

Steele ef al.

1981, 1983

Alexander et al.

1984b, 1987

1986

Fltzhugh et al.

Moir

Ludwig 1979

Cooper et al. 1987

PItster et al. 1977

Steele et al. 198

Komarkova et at.
1988

C. rossil

J. communis

P. monogynus

A. lanulosa

C. Iragills

A. cordifolla

B. rapens

J. communis

S. oraophlius
F. idahoensis

F. Idahoens/s
p. nervosa

A. misar

O. chllensis

p. myrsiniles

P. virginiana

C. rubascans

A. cordilolla

S. racemosa

Viola nuttalliI

B. rapons

Bromus richardsonil

(undergrowth sperse)

Ptister el al. 1977

Alexander ot al.

1987

Fitzhugh et at.

Alexander et al.

$1984 b$

Devellce and

Fitzhugh et at.

1987

Ludwlg 1979

Muldavin et al. 1986

Cooper et al. 1987

Atexander 1986

1988

Pilster et al. 1977

Hess and

Alexender 1986

Pfister et et. 1977

Steele et at.

981,1983

Meuk and

Henderson 1984

1981, 1983

lexender el el.

$1984 b$ 



\begin{tabular}{cccccc}
$\begin{array}{c}\text { Habltat type or } \\
\text { community typa }\end{array}$ & $\begin{array}{c}\text { Location and } \\
\text { elevation (teet) }\end{array}$ & Site & $\begin{array}{c}\text { Succeealonal } \\
\text { etatus }\end{array}$ & $\begin{array}{c}\text { Tree } \\
\text { aeeoclateo }\end{array}$ & $\begin{array}{c}\text { Princlpal undergrowth } \\
\text { epeciae }\end{array}$ \\
\hline
\end{tabular}

$\vec{\omega}$

\begin{tabular}{|c|c|}
\hline $\begin{array}{l}\text { Ables concolorl } \\
\text { Acer glebrum H.T. } \\
\text { (A. concolor- } \\
\text { Pseudolsuge menzlesill } \\
\text { A. glabrum H.T.) } \\
\text { A. glabrum (Iyplc) phase } \\
\text { Berberls repens phase (AZ,NM) } \\
\text { Holodiscus dumosus phase (AZ,NM) } \\
\text { Pachistime myrsiniles phase } \\
\text { Riparlan phase (NM) }\end{array}$ & $\begin{array}{l}\text { Mounlalns of } \\
\text { New Mexico, } \\
\text { Arlzona, southern } \\
\text { Colorado }(8,000 \text { - } \\
9,800) \text {, and } \\
\text { soulhern Utah } \\
(7,400 \cdot 8,400) \\
)\end{array}$ \\
\hline
\end{tabular}

Acer grendidentelum H.T.

A. grandidentelum (lyple) pha

Mountains of
soulh-ceniral

and eastern

Arizona, and

New Mexico

$(6,500-8.500)$

Ables concolorl

Arclostephylos pelule H.T.

Mountalns of

Southern Utah
$(8,100 \cdot 8,500)$

Ables concolorl

Arctostephylos uve.urs/ H.T.

Mountalns of

Mexico ond

southern Colorado

$(7,900-9,500)$

Abies concolorf

Berberls repens H.T.

Mounlains of

$(5,700-9,600)$

列

symphoricerpos oreophlius phase

Ables concolorl
Cercocarpus ledilollus M.T.

Mountalns of

central and

$(7,000-9,400)$

Mountains of

Ables concolor

(Screo toresi)

northern and

Soulhwostern

Now Moxico, ond
southorn Colorado
$(\theta, 000.10 .000)$

Ables concolort

Jugiens mejor H.T.

Mountains of

soulhern New

Mexlco and sout

$(6,500 \cdot 7,000)$

Ables concolorl

outhern Ulah

$(7,000-9,000)$

Mountalns of

Ables concolor

Physocarpus melveceus H.T.

$5,000-7,000)$

Mounlains o

New Mexico,

And southern

Colorado

$(6,500-9,500)$

Q. gambell $\mathrm{H}$ T.

O. gembelli (1ypic) phase

Holodiscus dumosus phase (NM)

Fesluce arizonice phase (AZ,NM)

Muhienbergle virescens phase

(AZ,NM)

Ables concolorl
Robinle neomexicene H.T.

Mountalns of

astern Arlzona

and sourhwest

$(8,500 \cdot 9,000)$

Mountalns of

Ables concolor

ceniral and

southern Ulah

Mounlains of

northern New

Mexico and

soulhern

Colorado

Ables concolorf

Veccinium myrlilius H.T.

$(8,500 \cdot 9,200)$

Ablos concolorl

Elymus iflicoldes H.T.

A. concolor

Pseudorsuga menziesiti

Ables concolorl

Fostuco afizonice H.T.

IA. concolor

Pseudotsuga monzlosill Poa londieriona H.T.]

F. orizonica (typlc) phase

Quercus gambelli phaso

P. fendiorlana phase

Cool molst
to well-
dratned

bies concolor series

$\begin{array}{ll}\text { A. concolor } & \text { P. menziosil } \\ \text { co.climax wilh } & \text { P. pungens } \\ \text { P. menzlesll. } & \text { P. engelmennil } \\ \text { Picee engelmennll } & \text { P. strobllormis } \\ \text { Plcee pungens } & \text { P. ponderose } \\ \text { P. ponderosa } & \text { P. llexills } \\ \text { P. slrobitormis } & \text { P. iremuloides } \\ \text { minor cllmaxes } & \\ \text { In some phases } & \end{array}$

lo well.
drained

A. concolor

cllmax or

co.climax with
P. menziesil.

P. engelmennll

minor cllmaxes

strobilormis

P. pondorosa

concolor

cllmax

Warm dn

concolor

A.climax wlith
P. menzlesil

Cool dry

A. concolor

cllmax

cool dry

menziesll

P. pungens
P. ponderose

P. Hexills

Pinus longaevo

P. menzles II

P. ponderose

P. flexllls

P. Iremuioldes

P. monzles"

P. pungens

A. grandis
P. ponderoso

P. contorto

P. llexills

P. Iremuloldes

A. concolor

P. menziesll

P. ponderose
P. Hexills

J. scopulorum

A. concolor

P. menzlesli

P. monxios/l
P. strobllormis

Probllorm/s
ponderosa

and
P. Hoxllis

Warm mols1

A. concolor

climax.

P. menzies/l

P. ponderose

P. Iremuloldes

Gellum mexicenum

$\begin{array}{ll}\text { Frexinus pennsyivenica } & \text { T. lendient } \\ \text { A. negundo } & \text { V. erizonice }\end{array}$

P. menzlesil

P. pungens

. llexills

scopulorum

P. tremuloides

P. menziesh

A. grandis

P. tremuloides

co.cllmax with

P. menziesll

Premuloides

A. concolor

Warm on

10 well.

co.cllmax wilh

P. menziesil.

P. strobllormis

minor cllmaxes

In some phases

P. menziesll

P. ponderose

P. strobllormis
P. Hexllis

P.

P. engelmennl

J. doppeene

P. tremuloldes

A. giabrum

A. ainllollo

A. tenulloll

H. dumosus

P. myrsiniles
P. virginiana

o. gambelli

clllatus

amplaxicaulls

r. Iendlerl

A. grendidentelum

J. malor

J. major

c. toonea

T. Iondien

Viola canadensis

A. pelule

B. repens
J. communts

J. communis

Q. gambellI

S. oreophilus

A. uve-ursi

P. myrsinites

J. communis
F. ovells

(F. virginione)

8. repens

J. communis

R. woodsll

S. oreophlius

Lethyrus leucanthus

Osmorhize spp.

C. ledillolius

A. repens

Q. gembell

S. oreophllus

H. dumosus

Ribes spp.

B. clllerus

(K. macrantha)

J. major

Q. gembellI

J. communts

B. repens

R. woodshl

S. oreophilus
C. rossil

P. malveceus

A. einillolle

Q. gembelli

Miliella slauropetale

S. recemose

Q. gembelli

B. repens

H. dumosus

S. oreophllus

intonice

M. duble

Lethyrus ens

Lethyrus arizonicus
$T$. lendiarl

Alexander el al.

$1984 a, 1987$
DeVellce el al.

1986

DeVellce and

Flizhugh el al.

1987

Molr and

Ludwlg 1979

Muldavin el al.

1986

Youngblood an

Mauk 1985

Alexander et al.

isc4a

DeVellce and

Ludwlg 1983
Filzhugh et al.

1987

Ludwig 1979

Muldavin ol al.

1988

Youngblood an
Mauk 1985

Develice el al.

1986

Mauk and

Henderson 1984

Youngbtood and

Mauk 1985

Youngblood and

Mauk 1985

Devellce ol at.

1986
tizhugh et al.

1987
1 izhugh ot al.

Alexander et at.

1984a

1 Fitzhugh el al.
1987

uldavin et al.

Youngblood and

Mauk 1985

Mauk and

Henderson 1984

Youngblood ano

Mauk 1985

Alexander el al.

1984a, 1987

Devellce et al.
1986

DeVellice and

Ludwig 1983

1987

Molr and

Ludw/9 1979

1986

Youngblood and

Filzhugh et al.

1987 



\begin{tabular}{|c|c|c|c|c|c|c|}
\hline $\begin{array}{l}\text { Habltal lype or } \\
\text { communlty type }\end{array}$ & $\begin{array}{l}\text { Location and } \\
\text { elevation (18et) }\end{array}$ & sile & $\begin{array}{l}\text { Succerstonal } \\
\text { status }\end{array}$ & $\begin{array}{c}\text { Tree } \\
\text { aesoclatee }\end{array}$ & $\begin{array}{l}\text { Princtpal undergrowth } \\
\text { specles }\end{array}$ & Authorliy \\
\hline $\begin{array}{l}\text { Ables concolorl } \\
\text { Muhlenbergle virescens H.T. }\end{array}$ & $\begin{array}{l}\text { Mountalns of } \\
\text { eastern Arlzona } \\
\text { and southwestern } \\
\text { New Mexlco } \\
(8,000 \cdot 9,200)\end{array}$ & Warm dry & $\begin{array}{l}\text { A. concolor } \\
\text { co.cllmax with } \\
\text { P. menziesil }\end{array}$ & $\begin{array}{l}\text { P. menzlesil } \\
\text { P. strobilorm/s } \\
\text { P. ponderose } \\
\text { P. tremuloldes }\end{array}$ & $\begin{array}{l}\text { M. virescens } \\
\text { O. gambelll } \\
\text { B. cllelus } \\
\text { P. lendleriena } \\
\text { C. rossil } \\
\text { L. erizonicus } \\
\text { Senecio spp. }\end{array}$ & $\begin{array}{l}\text { Fllzhugh ot al. } \\
1987\end{array}$ \\
\hline $\begin{array}{l}\text { Abies concolorl } \\
\text { Cerex loenee H.T. }\end{array}$ & $\begin{array}{l}\text { Mountelns of } \\
\text { eastern and } \\
\text { south.centrel } \\
\text { Artzone } \\
(8,600 \cdot 9,500)\end{array}$ & $\begin{array}{l}\text { Warm molst } \\
\text { to well. } \\
\text { dralned }\end{array}$ & $\begin{array}{l}\text { A. concolor } \\
\text { cllmax }\end{array}$ & $\begin{array}{l}\text { P. menzleell } \\
\text { P. ponderosa } \\
\text { P. strobllormis } \\
\text { P. Iremuloldes }\end{array}$ & $\begin{array}{l}\text { C. loenea } \\
\text { B. clllatus } \\
\text { P. pretensis } \\
\text { G. richerdsonll } \\
\text { fregerle spp. }\end{array}$ & $\begin{array}{l}\text { DeVellce and } \\
\text { Ludwig } 1983 \\
\text { Molr and } \\
\text { Ludwig } 1979 \\
\text { Muldavin et al. } \\
1986\end{array}$ \\
\hline $\begin{array}{l}\text { Ables concolorl } \\
\text { Erigeron eximius H.T. } \\
\text { [A. concolor- } \\
\text { Pseudorsuge menziesil } \\
\text { E. superbus H.T.] }\end{array}$ & $\begin{array}{l}\text { Mounteins of } \\
\text { northern and } \\
\text { southwestern New } \\
\text { Mexico, south. } \\
\text { centrel end } \\
\text { eastern Arlzona, } \\
\text { and Colorado } \\
(8,500 \cdot 9,800)\end{array}$ & Cool molst & $\begin{array}{l}\text { A. concolor } \\
\text { co.cltmax with } \\
\text { P. menzlesil. } \\
\text { P. engelmennll } \\
\text { P. pungens } \\
\text { minor cllmaxes }\end{array}$ & $\begin{array}{l}\text { P. menzleshl } \\
\text { P. engelmennll } \\
\text { P. pungens } \\
\text { P. ponderose } \\
\text { P. strobllormis } \\
\text { P. flexills } \\
\text { P. tremuloldes }\end{array}$ & $\begin{array}{l}\text { E. eximlus } \\
\text { (E. superbus) } \\
\text { B. cllerus } \\
\text { C. loenee } \\
\text { F. ovells } \\
\text { (F. virginlane) } \\
\text { H. perryl } \\
\text { L. arlzonicus }\end{array}$ & $\begin{array}{l}\text { DeVellce ot at. } \\
\text { 1986 } \\
\text { Filzhugh et al. } \\
1987 \\
\text { Moir and } \\
\text { Ludwlg } 1979 \\
\text { Muldavin et at. } \\
1986\end{array}$ \\
\hline $\begin{array}{l}\text { Ables concolorl } \\
\text { Gellum trillorum H.T. } \\
\text { (Rlperian forest) }\end{array}$ & $\begin{array}{l}\text { Mountelns of } \\
\text { northern New } \\
\text { Mexlco and } \\
\text { southern Cotoredo } \\
(7,500 \cdot 9,000)\end{array}$ & $\mathrm{CoOl} \mathrm{molst}$ & $\begin{array}{l}\text { A. concolor } \\
\text { cllmax }\end{array}$ & $\begin{array}{l}\text { P. menzles/l } \\
\text { J. scopulorum } \\
\text { P. engust/lolle }\end{array}$ & $\begin{array}{l}\text { G. trillorum } \\
\text { A. glebrum } \\
\text { A. tenullolle } \\
\text { P. virginiene } \\
\text { O. gambelli } \\
\text { P. pretens/s } \\
\text { T. tendlerl }\end{array}$ & $\begin{array}{l}\text { DeVellce et al. } \\
\quad 1986\end{array}$ \\
\hline $\begin{array}{l}\text { Abies concolor. } \\
\text { Pseudolsuge menziesill } \\
\text { Lalhyrus erlzonicus H.T. }\end{array}$ & $\begin{array}{l}\text { Mountalns of } \\
\text { norith-centrat } \\
\text { Arizona } \\
(8,000-10,000)\end{array}$ & Cool dry & $\begin{array}{l}\text { A. concolor } \\
\text { co.climax with } \\
\text { P. menziosil }\end{array}$ & $\begin{array}{l}\text { P. menziesil } \\
\text { P. ponderosa } \\
\text { P. tremuloides }\end{array}$ & $\begin{array}{l}\text { L. arizonicus } \\
\text { B. repens } \\
\text { G. richerdsonil }\end{array}$ & $\begin{array}{l}\text { Moir and } \\
\text { Ludwig } 1979\end{array}$ \\
\hline $\begin{array}{l}\text { Ables concolorl } \\
\text { Osmorhiza chllensis H.T. }\end{array}$ & $\begin{array}{l}\text { Mountains of } \\
\text { northern Utoh } \\
(5,400-7,000)\end{array}$ & $\begin{array}{l}\text { Warm molst } \\
\text { to well. } \\
\text { dralned }\end{array}$ & $\begin{array}{l}\text { A. concolor } \\
\text { cilmax }\end{array}$ & $\begin{array}{l}\text { P. menziesil } \\
\text { A. grandis } \\
\text { P. engelmannll } \\
\text { P. tramuloidas }\end{array}$ & $\begin{array}{l}\text { O. chllensis } \\
\text { A. ainilolle } \\
\text { P. malveceus } \\
\text { P. myrsinltes } \\
\text { P. virginlene }\end{array}$ & $\begin{array}{l}\text { Mauk and } \\
\quad \text { Henderson } 1984\end{array}$ \\
\hline $\begin{array}{l}\text { Ables concolorl } \\
\text { Sparse H.T. } \\
\text { [A. concolor. } \\
\text { Pseudolsuge menziesil H.T.] } \\
\text { Berberis repens phase } \\
\text { Robinia neomexicane phase }\end{array}$ & $\begin{array}{l}\text { Mountains of } \\
\text { northern end } \\
\text { southwestern New } \\
\text { Mexlco, south. } \\
\text { ceniral and } \\
\text { eastern Arizona, } \\
\text { and southern } \\
\text { Colorado } \\
(8,000 \cdot 9,800)\end{array}$ & Coot dry & $\begin{array}{l}\text { A. concolor } \\
\text { co.cllmax wilh } \\
\text { P. menzlesll. } \\
\text { P. pungens } \\
\text { P. angeimennll } \\
\text { minor cllmaxes }\end{array}$ & $\begin{array}{l}\text { P. menzles" } \\
\text { P. pungens } \\
\text { P. engelmennll } \\
\text { P. ponderose } \\
\text { P. strobllormis } \\
\text { P. llexills } \\
\text { P. erlstala } \\
\text { P. Iremuloides }\end{array}$ & $\begin{array}{l}\text { S. oreophllus } \\
\text { B. repens } \\
\text { O. gambelll } \\
\text { A. neomaxicane } \\
\text { B. cllatus } \\
\text { (Undergrowth sparse) }\end{array}$ & $\begin{array}{l}\text { Alexander et al. } \\
\text { 1984a } \\
\text { DeVellce et al. } \\
1986 \\
\text { DeVellce and } \\
\text { Ludwig 1983 } \\
\text { Fitzhugh et al. } \\
1987 \\
\text { Molr and } \\
\text { Ludwig } 1979 \\
\text { Muldavin ot at. } \\
1986\end{array}$ \\
\hline
\end{tabular}

Picea pungens series

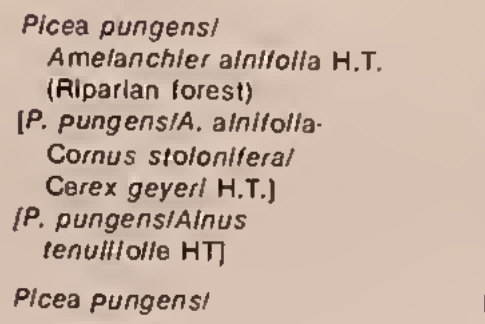

Mountalns of central and western Colorado

Mountalns of northern New Mexlco $(7,900 \cdot 9.200)$

Picee pungenst Berberis repens H.T. 8. repens (typic) phase
Symphoricarpos oreophilus phas

Mountalns o $7,800 \cdot 9,000)$

$\begin{array}{cl}\text { Picea pungensl } & \text { Mountains of } \\ \text { Cornus stolonllera H.T. } & \text { northern and } \\ \text { [P. Pungensl } & \text { southwestern } \\ \text { Swida sericee H.T.] } & \text { New Mexico, and } \\ \text { (Riparian lorest) } & \text { southern Colorado } \\ & (7,500 \cdot 9,200)\end{array}$

\begin{tabular}{|c|c|}
\hline $\begin{array}{l}\text { Picee pungensl } \\
\text { Juniperus communis H.T. }\end{array}$ & $\begin{array}{l}\text { Mountains of } \\
\text { central Utah } \\
(8,000 \cdot 8,600)\end{array}$ \\
\hline $\begin{array}{l}\text { Picee pungensl } \\
\text { Linnaea borealls H.T. } \\
\text { [P. pungans. } \\
\text { Pseudolsuga monzies/l H.T. } \\
\text { L. borealls phase] }\end{array}$ & $\begin{array}{l}\text { Mountains of } \\
\text { northern New } \\
\text { Mexico and } \\
\text { southern Colorado } \\
(8,200 \cdot 9,200)\end{array}$ \\
\hline $\begin{array}{l}\text { Pices pungens/ } \\
\text { Agropyron spicelum H.T. }\end{array}$ & $\begin{array}{l}\text { Mountalns of } \\
\text { northern Ulah } \\
(7,800-8,800)\end{array}$ \\
\hline $\begin{array}{l}\text { Picea pungensl } \\
\text { Fesiuce erizonice H.T. } \\
\text { [P. pungens/Carax loonea H.T. } \\
\text { Pinus ponderosa phase] }\end{array}$ & $\begin{array}{l}\text { Mounteins of } \\
\text { northern ond south. } \\
\text { weslern New Mexico, } \\
\text { easlern Arlzona, } \\
\text { and southern and } \\
\text { western Colorado } \\
(8,200-9,800)\end{array}$ \\
\hline
\end{tabular}

\begin{tabular}{|c|c|c|}
\hline Warm molst & $\begin{array}{l}\text { P. pungens } \\
\text { cllmax }\end{array}$ & $\begin{array}{l}\text { P. menzlesil } \\
\text { A. laslocarpa } \\
\text { P. ponderosa } \\
\text { P. Iremuloides } \\
\text { P. engustllolla }\end{array}$ \\
\hline Warm dry & $\begin{array}{l}\text { P. pungens } \\
\text { co.cllmax wilh } \\
\text { P. menzles II } \\
\text { A. concolor }\end{array}$ & $\begin{array}{l}\text { P. menziesli } \\
\text { A. concolor } \\
\text { P. ponderosa } \\
\text { P. llexllis } \\
\text { P. tremuloides }\end{array}$ \\
\hline Cool dry & $\begin{array}{l}\text { P. pungens } \\
\text { citmax. } \\
\text { P. menziesill } \\
\text { minor climax }\end{array}$ & $\begin{array}{l}\text { P. menziesll } \\
\text { P. contorte } \\
\text { P. ponderose } \\
\text { P. Hexills } \\
\text { J. scopulorum } \\
\text { P. tremuloldes }\end{array}$ \\
\hline Warm molst & $\begin{array}{l}\text { P. pungens } \\
\text { co.cllmax with } \\
\text { P. menzles/l }\end{array}$ & $\begin{array}{l}\text { P. menzlesll } \\
\text { A. concolor } \\
\text { P. tremuloides } \\
\text { P. angus } 1 \text { lollo }\end{array}$ \\
\hline
\end{tabular}

Cool dry

P. pungens

Cool molst

to well.

dralned

Warm very

dry

Warm dry

$P$. pungens climax (CO) or co.cllmax wilh P. manzlesll

(AZ,NM).

A. concolor P. ponderosa minor cllmaxes

P. pond

J. scopulorum
P. tremuloldos

A.

P. strobllorm

P. menzlesil

P. ponderose

J. scopulorum

P. menzies"l

P. ponderos

P. aristata (CO)

P. tromuloides
A. oinliolla

A. tenullolla
C. stoloniler

C. Siolonilera
(Swida sericea)

R. woodsil

Festuce thurberl

C. geyerl

A. uve-urs!

J. communts
B. clllalus

B. clllalus

(F. virgintane)

S. siellata

8. repens

J. communis

P. myrsintles

Ribes montlgenum

S. oreophllus
Aquillegla coerule

Aquilegia ceerula
Pyrolo sacundo

C. stolonilera

(S. sericea)

A. giabrum

A. tenullolle

8. repens

Calemegrosils canedensis

C. loenee

G. trillorum

G. richardsonil

S. stellate

J. communts

A. uve.ursl
B. repens

P. myrsiniles

L. borealls

P. myrsiniles

R. parvillorus

V. myrtillus
C. loenes

C. Ioenee

(E. superbus)

A. spicalum

B. repens

J. communis

P. myrsinites

S. oreophilus

F. arizonica

C. loenea

A. Irigide

Erigeron spp.

Fragerle spp.

Seneclo SpP.
Hess and Wasser 1982

Holiman 1988

1988

Develice et al.

1986

Mauk and

Henderson 198

Pflster 1972

Youngblood and
Mauk 1985

Alexander et al.

1987
DeVellce et al.

1986

DeVelice el al.

1986

Ludwlg 1979

Mauk and

Henderson 1984

DeVellce et al.

1986

Komarkova et al.

1988

Fitzhugh et al.

1987

Molr end

Ludwlg 1979 



\begin{tabular}{|c|c|c|c|c|c|c|}
\hline $\begin{array}{l}\text { Habllat typa or } \\
\text { community typo }\end{array}$ & $\begin{array}{l}\text { Locatlon and } \\
\text { slavation (faat) }\end{array}$ & Site & $\begin{array}{l}\text { Succaaalonal } \\
\text { atatua }\end{array}$ & $\begin{array}{c}\text { Traa } \\
\text { saaociatea }\end{array}$ & $\begin{array}{l}\text { Principal undergrowth } \\
\text { spaclaa }\end{array}$ & Authority \\
\hline $\begin{array}{l}\text { Picee pungens/ } \\
\text { Poe pratensis H.T. } \\
\text { (Riparlan loresl) }\end{array}$ & $\begin{array}{l}\text { Mountalns of } \\
\text { northarn end soulh. } \\
\text { wastern Naw Mexlco, } \\
\text { aastarn Arlzona, and } \\
\text { southern Coloredo } \\
(8,000-9,100)\end{array}$ & $\begin{array}{l}\text { Warm lo } \\
\text { cool molst }\end{array}$ & $\begin{array}{l}\text { P. pungens } \\
\text { cllmax or } \\
\text { co.cllmax wilh } \\
\text { P. menziesil. } \\
\text { A. . lesiocerpa } \\
\text { minor climex }\end{array}$ & $\begin{array}{l}\text { P. menziesil } \\
\text { A. leslocarpa } \\
\text { A. concolor } \\
\text { P. ponderose } \\
\text { P. strobllormis } \\
\text { P. tremuloldes }\end{array}$ & $\begin{array}{l}\text { P. prelensis } \\
\text { E. eximius } \\
\text { (E. superbus) } \\
\text { O. richardsonil } \\
\text { F. ovellis } \\
\text { (F. virginiane) }\end{array}$ & $\begin{array}{l}\text { Davellce ot al. } \\
1986 \\
\text { Fllzhugh al al. } \\
1987 \\
\text { Moli and } \\
\text { Ludwig } 1979\end{array}$ \\
\hline $\begin{array}{l}\text { Picee pungensI } \\
\text { Poe spp. H.T. } \\
\text { (Not riparlan) }\end{array}$ & $\begin{array}{l}\text { Mounlains of } \\
\text { north-cantrel } \\
\text { Coloredo } \\
(6,500-8,000)\end{array}$ & $\begin{array}{l}\text { Warm to } \\
\text { wall. } \\
\text { drelned }\end{array}$ & $\begin{array}{l}\text { P. pungens } \\
\text { cllmax }\end{array}$ & $\begin{array}{l}\text { Usually pura slands. } \\
\text { Mey conlain } \\
\text { P. monziesil } \\
\text { P. } \| \text { iemuloldes }\end{array}$ & $\begin{array}{l}\text { Poe spp. } \\
\text { A. ainilolle } \\
\text { Aose spp. } \\
\text { Sellx spp. }\end{array}$ & $\begin{array}{l}\text { Hoffman and } \\
\text { Alaxandar } 1983\end{array}$ \\
\hline $\begin{array}{l}\text { Picee pungens/ } \\
\text { Carex loeneo H.T. } \\
\text { [P. pungens/C. loenee H.T. } \\
\text { Pseudolsuge menziesili phase] }\end{array}$ & $\begin{array}{l}\text { Mounlains of } \\
\text { northern and } \\
\text { eastern Arlzone. } \\
\text { northarn and } \\
\text { soulhwestern New } \\
\text { Mexlco, and } \\
\text { soulharn Coloredo } \\
(8,000 \cdot 9,500)\end{array}$ & $\begin{array}{l}\text { Cool molsl } \\
\text { lo wall. } \\
\text { dralned }\end{array}$ & $\begin{array}{l}\text { P. pungens } \\
\text { co.cllmax with } \\
\text { P. menziesil. } \\
\text { A. concolor } \\
\text { P. engeimennil } \\
\text { minor cllmaxes }\end{array}$ & $\begin{array}{l}\text { P. menziesll } \\
\text { A. concolor } \\
\text { P. engelmennll } \\
\text { P. pondelose } \\
\text { P. strobllormis } \\
\text { P. Hexills } \\
\text { P. tremuloides }\end{array}$ & $\begin{array}{l}\text { C. loenee } \\
\text { B. ropens } \\
\text { F. arizonice } \\
\text { M. montent } \\
\text { B. cllietus } \\
\text { Festuce spp. } \\
\text { F. ovells } \\
\text { (F. Virginiene) }\end{array}$ & $\begin{array}{l}\text { Alaxander et al. } \\
1997 \\
\text { DaVallce ol al. } \\
1986 \\
\text { Filzhugh al al. } \\
1987 \\
\text { Molr and } \\
\text { Ludwig } 1979\end{array}$ \\
\hline $\begin{array}{l}\text { Picee pungensI } \\
\text { Arnice cordilolie H.T. } \\
\text { (RIparlan foresI) }\end{array}$ & $\begin{array}{l}\text { Front Renga, } \\
\text { north-cenlral } \\
\text { Colorado } \\
(7,500 \cdot 9,000)\end{array}$ & Cool molsl & $\begin{array}{l}\text { P. pungens } \\
\text { climax }\end{array}$ & $\begin{array}{l}\text { P. menzlesil } \\
\text { A. lesiocerpa } \\
\text { P. ponderosa } \\
\text { P. contorte } \\
\text { P. tromuloides }\end{array}$ & $\begin{array}{l}\text { A cordlolle } \\
\text { J. communis } \\
\text { A. woodsil } \\
\text { C. cenadensis } \\
\text { S. stellela }\end{array}$ & $\begin{array}{l}\text { Hess and } \\
\text { Alexandar } 1988\end{array}$ \\
\hline $\begin{array}{l}\text { Picee pungensl } \\
\text { Equiselum orvense H.T. }\end{array}$ & $\begin{array}{l}\text { Mountains of } \\
\text { southern Utah } \\
(8,000 \cdot 9,000)\end{array}$ & Warm molsl & $\begin{array}{l}\text { P. pungens } \\
\text { cilmax. } \\
\text { P. engeimennil } \\
\text { minor cllmax }\end{array}$ & $\begin{array}{l}\text { P. engelmannll } \\
\text { P. menzlesil } \\
\text { P. tremuloides }\end{array}$ & $\begin{array}{l}\text { E. arvense } \\
\text { G. ficherdsonll } \\
\text { O. chilensis } \\
T \text {. lendien }\end{array}$ & $\begin{array}{l}\text { Youngblood and } \\
\text { Mauk } 1985\end{array}$ \\
\hline $\begin{array}{l}\text { Picee pungensI } \\
\text { Erigeron eximius H.T. } \\
\text { P. pungens- } \\
\text { Picee engelmennill } \\
\text { E. superbus H.T.| } \\
\text { E. eximius (Iyplc) phese } \\
\text { Pinus ponderose phasa (AZ,NM) }\end{array}$ & $\begin{array}{l}\text { Mounlalns of } \\
\text { northern and } \\
\text { soulhwastern Naw } \\
\text { Maxlco, eastarn } \\
\text { Arlzone, and } \\
\text { soulhern Colorado } \\
(8,000 \cdot 9,800)\end{array}$ & $\begin{array}{l}\text { Cool molst } \\
\text { to well. } \\
\text { drained }\end{array}$ & $\begin{array}{l}\text { P. pungens } \\
\text { co.cllmax with } \\
\text { A. concolor } \\
\text { P. menzlesil } \\
\text { P. engelmennll. } \\
\text { A. leslocerpa } \\
\text { minor climax }\end{array}$ & $\begin{array}{l}\text { A. concolor } \\
\text { P. menziesil } \\
\text { P. engelmannil } \\
\text { A. leslocerpe } \\
\text { P. ponderose } \\
\text { P. strobilormis } \\
\text { P. flexills } \\
\text { P. llemuloides }\end{array}$ & $\begin{array}{l}\text { E. eximius } \\
\text { (E. superbus) } \\
\text { B. clliotus } \\
\text { C. loenes } \\
\text { G. richerdsonll } \\
\text { F. vesca } \\
\text { (F. americene) } \\
\text { H. perryi } \\
\text { T. lendierl }\end{array}$ & $\begin{array}{l}\text { Devellce et al. } \\
1986 \\
\text { Filzhugh et al. } \\
1987 \\
\text { Molr and } \\
\text { Ludwig } 1979\end{array}$ \\
\hline $\begin{array}{l}\text { Plcee pungensl } \\
\text { Fregeria ovalis H.T. }\end{array}$ & $\begin{array}{l}\text { Mountalns of } \\
\text { soulhern Naw } \\
\text { Mexlco } \\
(7,500 \cdot 9.800)\end{array}$ & Cool molst & $\begin{array}{l}\text { P. pungens } \\
\text { co.cllmax wlth } \\
\text { P. menziesil. } \\
\text { A. concolor } \\
\text { P. engelmennill } \\
\text { minor cllmexes }\end{array}$ & $\begin{array}{l}\text { P. menzles/l } \\
\text { A. concolor } \\
\text { P. engelmennll } \\
\text { P. strobllormis } \\
\text { P. ponderose } \\
\text { P. tremuloldes }\end{array}$ & $\begin{array}{l}\text { F. ovells } \\
\text { (F. virginiene) } \\
\text { A. giebrum } \\
\text { H. dumosus } \\
\text { B. richerdsonll } \\
\text { P. pratensis } \\
\text { F. vesce } \\
\text { (F. emericene) }\end{array}$ & $\begin{array}{l}\text { Alexander et al. } \\
\text { 1984a }\end{array}$ \\
\hline $\begin{array}{l}\text { Picea pungensl } \\
\text { Senecio cerdemine H.T. } \\
\text { [P. pungens- } \\
\text { Picea engeimannIII } \\
\text { S. cardamine H.T.] }\end{array}$ & $\begin{array}{l}\text { Mountalns of } \\
\text { aastarn Arizona } \\
\text { and southwestarn } \\
\text { Now Mextco } \\
(8,800-9,200)\end{array}$ & Cool molst & $\begin{array}{l}\text { P. pungens } \\
\text { co.cllmax with } \\
\text { A. leslocerpa } \\
\text { P. engelmannll } \\
\text { A. concolor } \\
\text { P. menzles }\end{array}$ & $\begin{array}{l}\text { A. lesiocerpe } \\
\text { P. engelmennll } \\
\text { P. menziesil } \\
\text { A. concolor } \\
\text { P. ponderosa } \\
\text { P. stroblrormis } \\
\text { P. tromulaldos }\end{array}$ & $\begin{array}{l}\text { S. coradmine } \\
\text { F. ovells } \\
\text { (F. virginiane) } \\
\text { G. richerdsonil } \\
\text { Helenium hoopesil } \\
\text { Preridium aqullinum } \\
\text { V. cansdens/s }\end{array}$ & $\begin{array}{l}\text { Flizhugh et al. } \\
1987 \\
\text { Molr and } \\
\text { Ludwig } 1979\end{array}$ \\
\hline $\begin{array}{l}\text { Picea pungens- } \\
\text { Pseudolsuge menzies/l H.T. } \\
\text { Afclostephylos uve-urs i phase } \\
\text { Juniperus communis phase } \\
\text { Velerlana acutlioba phase }\end{array}$ & $\begin{array}{l}\text { Mountalns ol } \\
\text { New Mexico, } \\
\text { Arlzona, and } \\
\text { southern Coloredo } \\
(7,800-9.500)\end{array}$ & $\begin{array}{l}\text { Warm dry } \\
\text { to well- } \\
\text { dralned }\end{array}$ & $\begin{array}{l}\text { P. pungens } \\
\text { co-cllmax wilh } \\
\text { P. menziesil. } \\
\text { P. engeimennll } \\
\text { minor cllmax } \\
\text { In some phases }\end{array}$ & $\begin{array}{l}\text { P. menziesll } \\
\text { A. concolor } \\
\text { P. engelmennill } \\
\text { P. strobilormis } \\
\text { P. ponderose } \\
\text { P. tremuloides }\end{array}$ & $\begin{array}{l}\text { A. uva-ursi } \\
\text { J. communis } \\
\text { P. myrsinites } \\
\text { E. eximius } \\
\text { (E. superbus) } \\
\text { L. arizonicus } \\
\text { V. eculliobo }\end{array}$ & $\begin{array}{l}\text { Molr and } \\
\text { Ludwlg } 1979\end{array}$ \\
\hline \multicolumn{7}{|c|}{ Abies grandis series } \\
\hline $\begin{array}{l}\text { Ables grandisI } \\
\text { Acer glabrum H.T. } \\
\text { A. glebrum (typlc) phase } \\
\text { Physocerpus malvecous phase }\end{array}$ & $\begin{array}{l}\text { Mountains of } \\
\text { central Idaho } \\
(3,800-6,400)\end{array}$ & Cool molsl & $\begin{array}{l}\text { A. grandis } \\
\text { cllmax. } \\
\text { A. lesiocerpo } \\
\text { minor cllmax }\end{array}$ & $\begin{array}{l}\text { A. lesiocerpe } \\
\text { P. menzles } / \\
\text { P. ponderose }\end{array}$ & $\begin{array}{l}\text { A. globrum } \\
\text { P. malvaceus } \\
\text { S. belulliolie } \\
\text { S. elbus } \\
\text { C. rubescens }\end{array}$ & Steele et al. 1981 \\
\hline $\begin{array}{l}\text { Ables grendisl } \\
\text { Linneea boreells H.T. } \\
\text { L. borealls (lyplc) phase } \\
\text { Veccinium globulare phase (ID) } \\
\text { Xerophyllum renex phase }\end{array}$ & $\begin{array}{l}\text { Mounlalns of } \\
\text { northern }(2,200- \\
5.200) \text { and cantral } \\
\text { Idaho, and southern } \\
\text { Montana } \\
(3.400-5.600)\end{array}$ & $\begin{array}{l}\text { Warm molst } \\
\text { to wall. } \\
\text { dralned }\end{array}$ & $\begin{array}{l}\text { A. grendis } \\
\text { climax. } \\
\text { A. tesiocerpe } \\
\text { minor cllmax } \\
\text { in some phases }\end{array}$ & $\begin{array}{l}\text { A. lesiocerpe } \\
\text { P. menzies } i l \\
\text { P. engeimennil } \\
\text { P. contorte } \\
\text { P. ponderose } \\
\text { L. occldentalls }\end{array}$ & $\begin{array}{l}\text { L. boreelis } \\
\text { A. einllolie } \\
\text { V. globulere } \\
\text { C. rubescens } \\
\text { A. cordllollo } \\
\text { Lupinus spp. } \\
\text { X. tenex }\end{array}$ & $\begin{array}{l}\text { Coopar el al. } 1987 \\
\text { Pflsler ot al. } 1977 \\
\text { Slaale et al. } 1981\end{array}$ \\
\hline $\begin{array}{l}\text { Ables grandis' } \\
\text { Pachistime myrsinites H.T. }\end{array}$ & $\begin{array}{l}\text { Mountalns of } \\
\text { northern Idaho } \\
\text { and eastern } \\
\text { Washinglon } \\
(2,600-4,900)\end{array}$ & $\begin{array}{l}\text { Cool dry } \\
\text { to well. } \\
\text { dralned }\end{array}$ & $\begin{array}{l}\text { A. grand/s } \\
\text { cllmax }\end{array}$ & $\begin{array}{l}\text { P. menzies II } \\
\text { P. engelmennil } \\
\text { P. contorte } \\
\text { P. ponderose } \\
\text { Pinus monticoie } \\
\text { L. occidentells }\end{array}$ & $\begin{array}{l}\text { P. myrsinites } \\
\text { L. borealls } \\
\text { Bromus vilgarls } \\
\text { G. trillorum } \\
\text { S. stellole } \\
\text { T. occidentele }\end{array}$ & $\begin{array}{l}\text { Daubenmlre and } \\
\text { Daubanmlre } 1968\end{array}$ \\
\hline $\begin{array}{l}\text { Ables grandist } \\
\text { Physocarpus meiveceus H.T. } \\
\text { P. meiveceus (typlc) phase } \\
\text { Coplis occidentalis phase }\end{array}$ & $\begin{array}{l}\text { Mounlains of } \\
\text { norlhern Idaho } \\
(2,200 \cdot 5,300)\end{array}$ & War $m$ dry & $\begin{array}{l}\text { A. grendis } \\
\text { cllmax }\end{array}$ & $\begin{array}{l}\text { P. menziesil } \\
\text { P. contorte } \\
\text { P. ponderose } \\
\text { P. monticole } \\
\text { L. occidentelis }\end{array}$ & $\begin{array}{l}\text { P. moiveceus } \\
\text { A. giebrum } \\
\text { H. discolor } \\
\text { C. occidentells } \\
\text { O. chilensis } \\
\text { S. racemosa }\end{array}$ & Cooper at al. 1987 \\
\hline $\begin{array}{l}\text { Abies grandisl } \\
\text { Splraea belulliolle H.T. }\end{array}$ & $\begin{array}{l}\text { Mountalns of } \\
\text { northern and } \\
\text { central Idaho } \\
(4,300-8,400)\end{array}$ & Warm dry & $\begin{array}{l}\text { A. grand/s } \\
\text { cllmax }\end{array}$ & $\begin{array}{l}\text { P. menziesil } \\
\text { P. ponderose } \\
\text { P. tremuloides }\end{array}$ & $\begin{array}{l}\text { S. belulilolie } \\
\text { S. elbus } \\
\text { C. rubescens } \\
\text { A. cordilolie }\end{array}$ & $\begin{array}{l}\text { Coopar et ol. } 1987 \\
\text { Sleala ot al. } 1981\end{array}$ \\
\hline $\begin{array}{l}\text { Ables grandisl } \\
\quad \text { Veccinium caespllosum H.T. }\end{array}$ & $\begin{array}{l}\text { Mountalns of } \\
\text { central Idaho } \\
(4,600 \cdot 5,500)\end{array}$ & $\begin{array}{l}\text { Cool molst } \\
\text { to well. } \\
\text { dralned } \\
\text { frost } \\
\text { pockets }\end{array}$ & $\begin{array}{l}\text { A. grandis } \\
\text { cllmax. } \\
\text { A. lasiocerpa } \\
\text { minor cllmax }\end{array}$ & $\begin{array}{l}\text { A. lesiocerpa } \\
\text { P. menziesil } \\
\text { P. engeimannill } \\
\text { P. contorie } \\
\text { P. ponderosa } \\
\text { L. occidentells }\end{array}$ & $\begin{array}{l}\text { V. coespilosum } \\
\text { C. rubescons } \\
\text { C. geveri } \\
\text { F. ovalis } \\
\text { (F. virginiona) } \\
\text { T. occidentele }\end{array}$ & Staele el al. 1981 \\
\hline $\begin{array}{l}\text { Ables grandisl } \\
\text { Vaccinium giobulare H.T. }\end{array}$ & $\begin{array}{l}\text { Mounlalns of } \\
\text { northern and } \\
\text { central Idaho } \\
(4,500 \cdot 8,500)\end{array}$ & Cool molst & $\begin{array}{l}\text { A. grendis } \\
\text { cllmax. } \\
\text { A. lesiocarpa } \\
\text { minor cllmax }\end{array}$ & $\begin{array}{l}\text { A. leslocerpa } \\
\text { P. menziesil } \\
\text { P. engeimennll } \\
\text { P. ponderosa } \\
\text { P. contorte } \\
\text { L. occidentelis }\end{array}$ & $\begin{array}{l}\text { V. giobulare } \\
\text { Lonicere utahensis } \\
\text { S. betulifolle } \\
\text { C. rubescens } \\
\text { C. geyerl } \\
\text { C. rossil }\end{array}$ & $\begin{array}{l}\text { Cooper et al. } 1987 \\
\text { Sleele el al. } 1981\end{array}$ \\
\hline $\begin{array}{l}\text { Abies grandisl } \\
\text { Calamagrostis rubescens H.T. }\end{array}$ & $\begin{array}{l}\text { Mounlains of } \\
\text { cenlral Ideho } \\
(5,200 \cdot 8,100)\end{array}$ & Cool dry & $\begin{array}{l}\text { A. grendis } \\
\text { climax }\end{array}$ & $\begin{array}{l}\text { P. menzles } \\
\text { P. contorto } \\
\text { P. ponderose } \\
\text { L. occidentelis }\end{array}$ & $\begin{array}{l}\text { C. rubescons } \\
\text { S. befulliolla } \\
\text { C. geyeri } \\
\text { A. cordilolia }\end{array}$ & Steele at al. 1981 \\
\hline $\begin{array}{l}\text { Ablos grandis/ } \\
\text { A sarum caudalum H.T. } \\
\text { A. caudatum (Iypla) phaso } \\
\text { Monziosll farruginoa phaso } \\
\text { Taxis brovilolla phase }\end{array}$ & $\begin{array}{l}\text { Mounlains of } \\
\text { northern Idaho } \\
(2,200 \cdot 5,950)\end{array}$ & Werm molst & $\begin{array}{l}\text { A. grandis } \\
\text { cllmax. } \\
\text { A. tesiocarpa } \\
\text { may be minor } \\
\text { cllmex }\end{array}$ & $\begin{array}{l}\text { A. lasiocarpa } \\
\text { P. angeimennil } \\
\text { P. menzlesil } \\
\text { P. contorta } \\
\text { P. monticole } \\
\text { L. occidentalis }\end{array}$ & $\begin{array}{l}\text { A. caudatum } \\
\text { H. discolor } \\
\text { M. terruginaa } \\
\text { T. brevilolla } \\
\text { C. unillora } \\
\text { C. occidentalis }\end{array}$ & Cooper el el. 1987 \\
\hline
\end{tabular}





\begin{tabular}{|c|c|c|c|c|c|c|}
\hline $\begin{array}{l}\text { Habltat lype or } \\
\text { community type }\end{array}$ & $\begin{array}{l}\text { Location and } \\
\text { elavation (feet) }\end{array}$ & Site & $\begin{array}{l}\text { Successional } \\
\text { status }\end{array}$ & $\begin{array}{c}\text { Tree } \\
\text { assoclates }\end{array}$ & $\begin{array}{l}\text { Princtpal undergrowth } \\
\text { spectes }\end{array}$ & Authorlty \\
\hline $\begin{array}{l}\text { Abies grandis/ } \\
\text { Clintonia unifiora H.T. } \\
\text { C. uniflora (typlc) phase } \\
\text { Manzlesia lerruginea phase (ID) } \\
\text { Physocarpus malvaceus phase (ID) } \\
\text { Taxis brevilolie phase (ID) } \\
\text { Aralla nudiceulls phase (MT) } \\
\text { Xarophyllum tanax phese }\end{array}$ & $\begin{array}{l}\text { Mountalns of } \\
\text { northern Montana, } \\
\text { and norlhern and } \\
\text { central Idaho } \\
\text { 1) }(2,000 \cdot 6,100)\end{array}$ & Warm molst & $\begin{array}{l}\text { A. grandls } \\
\text { cllmax. } \\
\text { A. lasiocarpe } \\
\text { minor cllmax }\end{array}$ & $\begin{array}{l}\text { A. lasiocarpa } \\
\text { P. manziasil } \\
\text { P. angalmannil } \\
\text { P. contorta } \\
\text { P. ponderose } \\
\text { P. monticola } \\
\text { L. occldentalls }\end{array}$ & $\begin{array}{l}\text { C. unilfiore } \\
\text { A. glabrum } \\
\text { L. boraails } \\
\text { M. farruginas } \\
\text { P. malvacaus } \\
\text { T. bravifolia } \\
\text { V. globulare } \\
\text { B. vulgaris } \\
\text { A. nudicautis } \\
\text { G. triflorum } \\
\text { X. renax }\end{array}$ & $\begin{array}{l}\text { Cooper et al. } 1987 \\
\text { Pflster et al. } 1977 \\
\text { Steele et al. } 1981\end{array}$ \\
\hline $\begin{array}{l}\text { Ables grandis/ } \\
\text { Coplis occidantalls H.T. }\end{array}$ & $\begin{array}{l}\text { Mountains of } \\
\text { central Idaho } \\
(1,600 \cdot 6,000)\end{array}$ & Warm dry & $\begin{array}{l}\text { A. grandis } \\
\text { citmax }\end{array}$ & $\begin{array}{l}\text { P. engelmannil } \\
\text { P. menzlesil } \\
\text { P. contorta } \\
\text { P. ponderosa } \\
\text { L. occidantalls }\end{array}$ & $\begin{array}{l}\text { C. occidentalis } \\
\text { S. albus } \\
\text { V. giobulara } \\
\text { X. tanax }\end{array}$ & Steete et al. 1981 \\
\hline $\begin{array}{l}\text { Ables grandis/ } \\
\text { Seneclo irlanguieris H.T. }\end{array}$ & $\begin{array}{l}\text { Mountatns of } \\
\text { northern Idaho } \\
(2,600-4,600)\end{array}$ & War $m$ molst & $\begin{array}{l}\text { A. grandis } \\
\text { cllmax. } \\
\text { A. lasiocerpa } \\
\text { may be minor } \\
\text { cttmax }\end{array}$ & $\begin{array}{l}\text { A. lasiocarpa } \\
\text { P. menziesil } \\
\text { P. angaimannll } \\
\text { L. occidentalls }\end{array}$ & $\begin{array}{l}\text { S. Irlangularis } \\
\text { Alhyrlum Illix.famina } \\
\text { Clrceaa alpina } \\
\text { C. occidentalls } \\
\text { Trautvalfera carolinansis }\end{array}$ & Cooper et at. 1987 \\
\hline $\begin{array}{l}\text { Ables grandisl } \\
\text { Xerophyllum tenax H.T. } \\
\text { X. Ienax (typlc) phese } \\
\text { Vaccinium globulere phase (ID) } \\
\text { Coptis occldantalis phase (ID) }\end{array}$ & $\begin{array}{l}\text { Mountains of } \\
\text { northern and } \\
\text { central Idaho, } \\
\text { and northwestern } \\
\text { Montana } \\
(4,400-6,500)\end{array}$ & Cool dry & $\begin{array}{l}\text { A. grandis } \\
\text { cllmax. } \\
\text { A. lesiocerpo } \\
\text { minor climax }\end{array}$ & $\begin{array}{l}\text { A. lasiocarpe } \\
\text { P. menzlasil } \\
\text { P. angeimannil } \\
\text { P. contorta } \\
\text { P. ponderosa } \\
\text { L. occldentalls }\end{array}$ & $\begin{array}{l}\text { X. renax } \\
\text { P. myrsinites } \\
\text { V. giobulara } \\
\text { Veccinium scoperium } \\
\text { C. rubescens } \\
\text { Arnica latifolla } \\
\text { C. occidentalls }\end{array}$ & $\begin{array}{l}\text { Cooper et at. } 1987 \\
\text { Pftster et at. } 1977 \\
\text { Steele et al. } 1981\end{array}$ \\
\hline \multicolumn{7}{|c|}{ Thuja plicata series } \\
\hline $\begin{array}{l}\text { Thuia pllcarel } \\
\text { Oplopenax horridum H.T. }\end{array}$ & $\begin{array}{l}\text { Mountains of } \\
\text { Montana, northern } \\
\text { Idaho, and eastern } \\
\text { Washington } \\
(1.600-4,900)\end{array}$ & Cool molst & $\begin{array}{l}\text { T. pllcata } \\
\text { climax or } \\
\text { co.climax with } \\
\text { T. hetarophylla. } \\
\text { A. lasiocarpa } \\
\text { may be minor } \\
\text { climax }\end{array}$ & $\begin{array}{l}\text { T. helerophylla } \\
\text { A. lasiocarpa } \\
\text { P. menziesil } \\
\text { P. angeimannil } \\
\text { A. grandis } \\
\text { P. monilcole } \\
\text { L. occidenialls }\end{array}$ & $\begin{array}{l}\text { O. horridum } \\
\text { A. flilix-lamina } \\
\text { Dryopleris dilierate } \\
\text { S. triangularis } \\
\text { S. stellata } \\
\text { Streplopus amplaxilolius } \\
\text { Tlaralla spp. }\end{array}$ & $\begin{array}{l}\text { Cooper et al. } 1987 \\
\text { Daubenmire and } \\
\text { Daubenmlre } 1968 \\
\text { Pfister of al. } 1977\end{array}$ \\
\hline $\begin{array}{l}\text { Thuje plicatel } \\
\text { Pechistime myrsiniles H.T. }\end{array}$ & $\begin{array}{l}\text { Mountains of } \\
\text { northern Idaho } \\
\text { and eestarn } \\
\text { Washington } \\
(2,600 \cdot 4,700)\end{array}$ & $\begin{array}{l}\text { Coot dry } \\
\text { to wall. } \\
\text { dralned }\end{array}$ & $\begin{array}{l}\text { T. ollcale } \\
\text { climax }\end{array}$ & $\begin{array}{l}\text { P. engeimannil } \\
\text { P. menziesil } \\
\text { A. grandis } \\
\text { P. monticole } \\
\text { L. occidantalls }\end{array}$ & $\begin{array}{l}\text { P. myrsinites } \\
\text { A. glabrum } \\
\text { C. occidentalls } \\
\text { Disporum oregonum } \\
\text { G. triflorum } \\
\text { S. stellata }\end{array}$ & $\begin{array}{l}\text { Daubenmira and } \\
\text { Daubenmire } 1968\end{array}$ \\
\hline $\begin{array}{l}\text { Thuja pllcatal } \\
\text { Adientum pedatum H.T. }\end{array}$ & $\begin{array}{l}\text { Mountains of } \\
\text { northern Idaho } \\
(\leq 3,000)\end{array}$ & Cool molst & $\begin{array}{l}\text { T. plicele } \\
\text { climax. } \\
\text { r. haterophylia } \\
\text { minor climax }\end{array}$ & $\begin{array}{l}\text { T. hetarophylle } \\
\text { A. grendis } \\
\text { P. monticole } \\
\text { L. occidentelis }\end{array}$ & $\begin{array}{l}\text { A. pedatum } \\
\text { A. flilix lemina } \\
\text { C. unlifora } \\
\text { D. dilatata }\end{array}$ & Cooper et al. 1987 \\
\hline $\begin{array}{l}\text { Thuja plicelal } \\
\text { A sarum caudalum H.T. } \\
\text { A. caudarum (typic) phase } \\
\text { Menziesia rerruginea phase } \\
\text { Taxis brevifolla phase }\end{array}$ & $\begin{array}{l}\text { Mountalns of } \\
\text { northern Idaho } \\
(2,200-5,200)\end{array}$ & Warm molst & $\begin{array}{l}\text { T. olicalo } \\
\text { climax }\end{array}$ & $\begin{array}{l}\text { A. leslocarpe } \\
\text { P. engalmannl } \\
\text { A. grandis } \\
\text { P. menzlesll } \\
\text { P. contorta } \\
\text { P. monticola } \\
\text { P. pondorosa } \\
\text { L. occldontalls }\end{array}$ & $\begin{array}{l}\text { A. caudelum } \\
\text { A. glabrum } \\
\text { M. larruginos } \\
\text { T. orovifolia } \\
\text { C. occlidentalls } \\
\text { Polystichum munirum } \\
\text { P. aguilinum } \\
\text { viofa glabella }\end{array}$ & Cooper el al. 1987 \\
\hline $\begin{array}{l}\text { Thuje plicatal } \\
\text { Alhyrium tillx-femina H.T. } \\
\text { A. Illix-temina (typlc) phase } \\
\text { Adlantum pedalum phase (ID) }\end{array}$ & $\begin{array}{l}\text { Mountalns of } \\
\text { northern Idaho } \\
\text { and eastern } \\
\text { Washlngton } \\
(1,500 \cdot 4,700)\end{array}$ & $\begin{array}{l}\text { Warm molst } \\
\text { to wet }\end{array}$ & $\begin{array}{l}\text { T. plicala } \\
\text { climax or } \\
\text { co.cllmax with } \\
\text { T. helerophylla }\end{array}$ & $\begin{array}{l}\text { T. heterophylla } \\
\text { P. engelmannil } \\
\text { P. menzlesil } \\
\text { A. grandis } \\
\text { P. monticola }\end{array}$ & $\begin{array}{l}\text { A. Ililix-temina } \\
\text { A. pedatum } \\
\text { G. Irifiorum } \\
\text { S. iriangularis } \\
\text { S. amplexilolius }\end{array}$ & $\begin{array}{l}\text { Cooper et al. } 1987 \\
\text { Daubenmire and } \\
\text { Daubenmire } 1968\end{array}$ \\
\hline $\begin{array}{l}\text { Thuia pllcatel } \\
\text { Clinfonia uniflora H.T. } \\
\text { C. uniflora (typlc) phase } \\
\text { Menziesia ferruginea phase } \\
\text { Taxis breviltolla phase (ID) } \\
\text { Aralla nudicaulls phase (MT) } \\
\text { Xerophyllum tenex phase (ID) }\end{array}$ & $\begin{array}{l}\text { Mountains ol } \\
\text { northern Idaho } \\
\text { and northwestern } \\
\text { Montana } \\
(1.500 \cdot 5.500)\end{array}$ & $\begin{array}{l}\text { Warm dry } \\
\text { bottomlands }\end{array}$ & $\begin{array}{l}\text { T. plicata } \\
\text { cilmax. } \\
\text { A. lasiocarpa } \\
\text { A. grandis } \\
\text { T. heterophylla } \\
\text { minor cllmaxes }\end{array}$ & $\begin{array}{l}\text { A. lasiocarpa } \\
\text { A. grandis } \\
\text { T. heterophylle } \\
\text { P. engelmennll } \\
\text { P. menzlesil } \\
\text { P. contorla } \\
\text { L. occidentalis }\end{array}$ & $\begin{array}{l}\text { C. unifiora } \\
\text { L. boreells } \\
\text { M. terruginea } \\
\text { V. giobulere } \\
\text { T. brevilolla } \\
\text { A. nudicaulis } \\
\text { X. tenax }\end{array}$ & $\begin{array}{l}\text { Cooper et al. } 1987 \\
\text { Pfister et al. } 1977\end{array}$ \\
\hline $\begin{array}{l}\text { Thuia plicatel } \\
\text { Gymnocarplum dryopteris H.T. }\end{array}$ & $\begin{array}{l}\text { Mountains of } \\
\text { northern Idaho } \\
\{3.200-4.500)\end{array}$ & Cool dry & $\begin{array}{l}\text { T. plicafa } \\
\text { climax }\end{array}$ & $\begin{array}{l}\text { P. engeimannil } \\
\text { P. manzlesil } \\
\text { A. grandis } \\
\text { P. monticola }\end{array}$ & $\begin{array}{l}\text { G. dryoptaris } \\
\text { A. pedatum } \\
\text { A. IIIix.temina } \\
\text { C. uniliora }\end{array}$ & Cooper et al. 1987 \\
\hline \multicolumn{7}{|c|}{ Tsuga heterophylla series } \\
\hline $\begin{array}{l}\text { Isuga haterophyllal } \\
\text { Menziasia ferruginea H.T. }\end{array}$ & $\begin{array}{l}\text { Mountalns of } \\
\text { northern idaho } \\
( \pm 5,000)\end{array}$ & $\begin{array}{l}\text { Warm well- } \\
\text { dralned }\end{array}$ & $\begin{array}{l}\text { T. heterophylla } \\
\text { cllmax }\end{array}$ & $\begin{array}{l}\text { A. lasiocarpa } \\
\text { P. angeimennll }\end{array}$ & $\begin{array}{l}\text { M. lerruginea } \\
\text { V. globulare } \\
\text { X. lenex }\end{array}$ & Cooper el al. 1987 \\
\hline $\begin{array}{l}\text { Tsuga heterophylial } \\
\text { Pechistima myrsinites H.T. }\end{array}$ & $\begin{array}{l}\text { Mountains of } \\
\text { northern Idaho } \\
\text { and eastern } \\
\text { Washington } \\
(2,700-3,900)\end{array}$ & Cool moist & $\begin{array}{l}\text { T. heterophylla } \\
\text { cllmax }\end{array}$ & $\begin{array}{l}\text { T. pllcata } \\
\text { P. menzlesil } \\
\text { A. grandis } \\
\text { P. monticole } \\
\text { L. occidentalls }\end{array}$ & $\begin{array}{l}\text { P. myrsinites } \\
\text { L. boraalls } \\
\text { Vaccinilum membranaceum } \\
\text { C. unilore } \\
\text { G. dryopterls }\end{array}$ & $\begin{array}{l}\text { Daubenmire and } \\
\text { Daubenmire } 1968\end{array}$ \\
\hline $\begin{array}{l}\text { Tsuga heterophyllal } \\
\text { A sarum caudatum H.T. } \\
\text { A. caudelum (typlc) phase } \\
\text { Menzlasla lerruginea phase } \\
\text { A ralla nudicaulis phase }\end{array}$ & $\begin{array}{l}\text { Mountains of } \\
\text { northern Idaho } \\
(2,200-5.000)\end{array}$ & $\begin{array}{l}\text { Warm well. } \\
\text { drained }\end{array}$ & $\begin{array}{l}\text { T. hatarophylla } \\
\text { cllmax }\end{array}$ & $\begin{array}{l}\text { A. lesiocarpe } \\
\text { P. engaimannil } \\
\text { P. menziesil } \\
\text { A. grandis } \\
\text { P. monticole } \\
\text { P. contorta } \\
\text { L. occidentells } \\
\text { T. plicela }\end{array}$ & $\begin{array}{l}\text { A. caudatum } \\
\text { L. boreelis } \\
\text { M. ferruginea } \\
\text { P. myrsinifes } \\
\text { A. nudicaulis } \\
\text { C. unifiore } \\
\text { C. occidentalis } \\
\text { P. hookerl }\end{array}$ & Cooper et al. 1987 \\
\hline $\begin{array}{l}\text { Tsuga haterophyllal } \\
\text { Clintonia unifiora H.T. } \\
\text { C. Unifiora (typlc) phase } \\
\text { Manziesia ferruginas phase (ID) } \\
\text { Aralla nudicaulis phase } \\
\text { Xerophyllum tenax phase (ID) }\end{array}$ & $\begin{array}{l}\text { Mountains of } \\
\text { norlhern Idaho } \\
\text { and northwestern } \\
\text { Montana } \\
(2,000 \cdot 4,500)\end{array}$ & $\begin{array}{l}\text { Warm moist } \\
\text { to well. } \\
\text { dralned }\end{array}$ & $\begin{array}{l}\text { T. helerophylia } \\
\text { cllmax. } \\
\text { A. Iasiocarpa } \\
\text { A. grandis } \\
\text { r. plicele } \\
\text { minor cllmaxes }\end{array}$ & $\begin{array}{l}\text { A. lasiocarpa } \\
\text { A. grandis } \\
\text { T. olicata } \\
\text { P. engeimannil } \\
\text { P. menzlasil } \\
\text { P. contorta } \\
\text { P. monticola } \\
\text { L. occidenialls }\end{array}$ & $\begin{array}{l}\text { C. unifiora } \\
\text { M. ferruginea } \\
\text { T. brevifolie } \\
\text { V. globulare } \\
\text { V. membraneceum } \\
\text { A. nudicaulis } \\
X \text {. tanex }\end{array}$ & $\begin{array}{l}\text { Cooper et al. } 1987 \\
\text { Pflster et al. } 1977\end{array}$ \\
\hline $\begin{array}{l}\text { Isuga helerophylial } \\
\text { Gymnocarplum dryopteris H.T. }\end{array}$ & $\begin{array}{l}\text { Mountalns ol } \\
\text { northern ldaho } \\
(2,500 \cdot 4,500)\end{array}$ & $\begin{array}{l}\text { Warm dry } \\
\text { to well. } \\
\text { drelned }\end{array}$ & $\begin{array}{l}\text { T. hatarophylla } \\
\text { co.cllmax with } \\
\text { T. pllcata }\end{array}$ & $\begin{array}{l}\text { T. plicata } \\
\text { P. engeimannll } \\
\text { A. grandis } \\
\text { P. monticole } \\
\text { L. occldentalls }\end{array}$ & $\begin{array}{l}\text { G. dryopteris } \\
\text { A. caudatum } \\
\text { C. unifiora } \\
\text { C. occidentalls } \\
\text { S. stellata }\end{array}$ & Cooper et al. 1987 \\
\hline \multicolumn{7}{|c|}{ Pinus flexilis series } \\
\hline $\begin{array}{l}\text { Pinus Hexillsl } \\
\text { Arclostaphylos uve-urs/ H.T. }\end{array}$ & $\begin{array}{l}\text { Mountains of } \\
\text { northern New } \\
\text { Mexico and } \\
\text { southern Colorado } \\
(9,500 \cdot 10,000)\end{array}$ & Cool dry & $\begin{array}{l}\text { P. flexilis } \\
\text { co.climex with } \\
\text { P. menziesil. } \\
\text { P. engeimannil } \\
\text { minor climax }\end{array}$ & $\begin{array}{l}\text { P. menziesil } \\
\text { P. engeimann"l } \\
\text { P. tromuloides }\end{array}$ & $\begin{array}{l}\text { A. uve.ursi } \\
\text { J. communis }\end{array}$ & $\begin{array}{l}\text { Develice of al. } \\
1986\end{array}$ \\
\hline $\begin{array}{l}\text { Pinus liexillsl } \\
\text { Berberis repens H.T. }\end{array}$ & $\begin{array}{l}\text { Mountains of } \\
\text { northern Ulah } \\
(6,500-7,000)\end{array}$ & Werm dry & $\begin{array}{l}\text { P. flexllis } \\
\text { cllmex }\end{array}$ & $\begin{array}{l}\text { P. menziesil } \\
\text { J. scopuiorum }\end{array}$ & $\begin{array}{l}\text { B. rapens } \\
\text { P. myrsinites } \\
\text { P. virgintene } \\
\text { S. oreophllus } \\
\text { A. spicalum }\end{array}$ & $\begin{array}{l}\text { Meuk and } \\
\text { Henderson } 1984\end{array}$ \\
\hline
\end{tabular}





\begin{tabular}{|c|c|c|c|c|c|c|}
\hline $\begin{array}{l}\text { Habltat type or } \\
\text { community type }\end{array}$ & $\begin{array}{l}\text { Location and } \\
\text { alavation (faat) }\end{array}$ & Site & $\begin{array}{l}\text { Succeeelonal } \\
\text { statue }\end{array}$ & $\begin{array}{c}\text { Traa } \\
\text { aasoclataa }\end{array}$ & $\begin{array}{l}\text { Princlpal undergrowth } \\
\text { speclas }\end{array}$ & Authority \\
\hline $\begin{array}{l}\text { Plnus Hexilst } \\
\text { Carcocerpus ledilollus H.T. }\end{array}$ & $\begin{array}{l}\text { Mounleins ol } \\
\text { southeastern ldaho } \\
\text { and northern Utah } \\
(7,000-8,700)\end{array}$ & Warm dry & $\begin{array}{l}\text { P. Ilexills } \\
\text { cllmax or } \\
\text { co.cllmax with } \\
\text { P. menzles/l. } \\
\text { J. scopulorum } \\
\text { minor cllmex }\end{array}$ & $\begin{array}{l}\text { P. manziesll } \\
\text { J. scopulorum }\end{array}$ & $\begin{array}{l}\text { C. ledllollus } \\
\text { B. repans } \\
\text { S. oreophllus } \\
\text { A. spicalum } \\
\text { H. Kingll } \\
\text { B. seglltete }\end{array}$ & $\begin{array}{l}\text { Mauk and } \\
\text { Henderson } 1984 \\
\text { Steele al al. } 1983\end{array}$ \\
\hline $\begin{array}{l}\text { PInus llexil/s/ } \\
\text { Juniperus communis H.T. }\end{array}$ & $\begin{array}{l}\text { Mounleins of } \\
\text { Monlena }(4,600- \\
8,300) \text {, north. } \\
\text { waslarn Wyoming } \\
(7,000 \cdot 9,500) \text {, } \\
\text { southeesiern } \\
\text { Wyomlng, end } \\
\text { central and } \\
\text { western Coloredo } \\
(8,300-9,300)\end{array}$ & Warm dry & $\begin{array}{l}\text { P. llexills } \\
\text { cllmax. } \\
\text { P. manzles/l } \\
\text { P. contorte } \\
\text { P. albiceulls } \\
\text { minor cllmaxes }\end{array}$ & $\begin{array}{l}\text { P. menzlesil } \\
\text { P. contorta } \\
\text { P. elbicaulls } \\
\text { P. engalmannill } \\
\text { P. ponderosa } \\
\text { P. tremuloldes }\end{array}$ & $\begin{array}{l}\text { J. communls } \\
\text { A. uva.urst } \\
\text { S. cenedensis } \\
\text { C. purpurascens } \\
\text { C. rossll } \\
\text { A. cordllolle }\end{array}$ & $\begin{array}{l}\text { Alexander et al. } \\
1986 \\
\text { Hess and } \\
\text { Alexander } 1986 \\
\text { Hoffman } 1988 \\
\text { Hottman and } \\
\text { Alexander } 1980 \\
\text { Pllster el al. } 1977 \\
\text { Steele et al. } 1983\end{array}$ \\
\hline $\begin{array}{l}\text { Pinus Ilexills } \\
\text { Agropyron spicatum H.T. }\end{array}$ & $\begin{array}{l}\text { Mountains of } \\
\text { Monlana eest } \\
\text { of Continental } \\
\text { Olvide } \\
(4,400 \cdot 6,600)\end{array}$ & $\begin{array}{l}\text { Warm very } \\
\text { dry }\end{array}$ & $\begin{array}{l}\text { P. llexills } \\
\text { climax or } \\
\text { co-cllmax with } \\
\text { J. scopulorum. } \\
\text { P. ponderose } \\
\text { minor cllmax }\end{array}$ & $\begin{array}{l}\text { P. ponderose } \\
\text { J. scopulorum }\end{array}$ & $\begin{array}{l}\text { A. spicelum } \\
\text { e. grecills } \\
\text { H. kingll } \\
\text { K. cristete } \\
\text { (K. mecrentha) } \\
\text { Cerex spp. }\end{array}$ & Pilster et al. 1977 \\
\hline $\begin{array}{l}\text { Pinus } / \text { exills } \\
\text { Celemegrostis purpurascens H.T. }\end{array}$ & $\begin{array}{l}\text { Mounlains ol } \\
\text { north-cantrel } \\
\text { Colorado } \\
(9,700 \cdot 11,000)\end{array}$ & Cool dry & $\begin{array}{l}\text { P. } / \text { exills } \\
\text { climax }\end{array}$ & $\begin{array}{l}\text { Usually pure stends. } \\
\text { May conlain } \\
\text { P. engelmennll } \\
\text { P. contorte }\end{array}$ & $\begin{array}{l}\text { C. purpurascens } \\
\text { Aranarla lendlerl } \\
\text { Erigeron spp. } \\
\text { Pulsalilla ludoviciene }\end{array}$ & $\begin{array}{l}\text { Hess and } \\
\text { Alaxander } 1986\end{array}$ \\
\hline $\begin{array}{l}\text { Pinus /lex/lis/ } \\
\text { Festuca Idehoens/s H.T. } \\
\text { F. Idahoens/s (lyplc) phase } \\
\text { Festuce scebrelle phase (MT) }\end{array}$ & $\begin{array}{l}\text { Mountains of } \\
\text { southweslern } \\
\text { Montena, central } \\
\text { Idaho, and } \\
\text { northwestern } \\
\text { Wyoming } \\
(4,800-8,300)\end{array}$ & Warm dry & $\begin{array}{l}\text { P. Hexills } \\
\text { co-cllmax wlth } \\
\text { P. menzlesil. } \\
\text { J. scopulorum } \\
\text { minor cllmex }\end{array}$ & $\begin{array}{l}\text { P. menzlesh } \\
\text { J. scopulorum }\end{array}$ & $\begin{array}{l}\text { F. Idehoensis } \\
\text { A. irldentela } \\
\text { A. scipelum } \\
\text { H. kingll } \\
\text { F. scebrella } \\
\text { B. segllete }\end{array}$ & $\begin{array}{l}\text { Pflster et al. } 1977 \\
\text { Staele et al. } \\
\text { 1981, } 1983\end{array}$ \\
\hline $\begin{array}{l}\text { Pinus llexllis/ } \\
\text { Hesperochloa klngll H.T. }\end{array}$ & $\begin{array}{l}\text { Mounleins of } \\
\text { northwestern } \\
\text { and southeaslern } \\
\text { Wyoming } \\
(7,200-9,300)\end{array}$ & Werm dry & $\begin{array}{l}\text { P. llexills } \\
\text { cllmax or } \\
\text { co.cllmex with } \\
\text { P. menzlesil. } \\
\text { P. scopulorum } \\
\text { minor climax }\end{array}$ & $\begin{array}{l}\text { Usuelly pure stands } \\
\text { (SE WY). } \\
\text { Mey contain (NW WY) } \\
\text { P. menzlesil } \\
\text { J. scopulorum }\end{array}$ & $\begin{array}{l}\text { H. kingll } \\
\text { A. splcatum } \\
\text { K. cristata } \\
\text { C. ross } / \\
\text { A. } \mathrm{m} / \mathrm{ser} \\
\text { B. seglttata }\end{array}$ & $\begin{array}{l}\text { Alexander el al. } \\
\quad 1986 \\
\text { Sleele el al. } 1983\end{array}$ \\
\hline $\begin{array}{l}\text { Pinus Ilexills! } \\
\text { Saxllraga bronchialls H.T. } \\
{[P \text {. Ilexllisl }} \\
\text { Clliarla oustromontana H.T.] }\end{array}$ & $\begin{array}{l}\text { Mountalns of } \\
\text { south.centrel } \\
\text { Colorado } \\
(8,500-9,500)\end{array}$ & Cool dry & $\begin{array}{l}\text { P. Ilexills } \\
\text { cllmax }\end{array}$ & P. menzies & $\begin{array}{l}\text { S. bronchlalls } \\
\text { (C. austromontana) } \\
\text { J. communis } \\
\text { A. woodsll } \\
\text { S. oreophllus } \\
\text { F. thurbert }\end{array}$ & $\begin{array}{l}\text { Komarkova of al. } \\
1988\end{array}$ \\
\hline $\begin{array}{l}\text { Pinus llexills/ } \\
\text { rrilollum desyphyllum H.T. }\end{array}$ & $\begin{array}{l}\text { Mounlains of } \\
\text { north.central } \\
\text { Colorado } \\
(11,000-11,500)\end{array}$ & $\begin{array}{l}\text { Cool dry } \\
\text { to well. } \\
\text { drained }\end{array}$ & $\begin{array}{l}\text { P. } \| \text { iexils } \\
\text { cllmax }\end{array}$ & $\begin{array}{l}\text { Usually pure stands. } \\
\text { May conlain } \\
\text { P. engolmannll } \\
\text { A. laslocarpe }\end{array}$ & $\begin{array}{l}\text { T. desyphyllum } \\
\text { C. loenea } \\
\text { A. fend/eri } \\
\text { Merlensia virlais } \\
\text { Oreoxis alpina }\end{array}$ & $\begin{array}{l}\text { Hess and } \\
\text { Alexander } 1986\end{array}$ \\
\hline$-\cdots$ & & & & & $-\cdots$ & \\
\hline $\begin{array}{l}\text { Pinus llexllis- } \\
\text { PInus longaeva H.T. }\end{array}$ & $\begin{array}{l}\text { Mountalns of } \\
\text { central and } \\
\text { soulhern Uteh } \\
(9,000-10,200)\end{array}$ & Cool dry & $\begin{array}{l}\text { P. llexills } \\
\text { co.cllmax wilh } \\
\text { P. longaeva. } \\
\text { P. manzlesil } \\
\text { J. scopulorum } \\
\text { minor climexes }\end{array}$ & $\begin{array}{l}\text { P. longaeve } \\
\text { P. manzlasll } \\
\text { J. scopulorum } \\
\text { P. Iramuloldes }\end{array}$ & $\begin{array}{l}\text { S. oreophilus } \\
\text { B. repens } \\
\text { J. communis } \\
\text { A. patula } \\
\text { C. rossil } \\
\text { A. m/ser }\end{array}$ & $\begin{array}{l}\text { Youngblood and } \\
\text { Mauk } 1985\end{array}$ \\
\hline
\end{tabular}

Populus tremuloldes/

Acer glebrum C.T.

(Alparlan lorest)

Populus tremuloldas.

ablas laslocarpal

Amelanchlar alnilolla C.T.

talns ol

south-ceniral

$(0,000-9,200)$

Mountalns of

eastern Idaho

and Ulah

Mountains of

and soulheaster

$(5,600 \cdot 7,500)$

Pseudolsuga menziesill

Amelanchler alnillolla C.T.

\begin{tabular}{|c|c|c|}
\hline Warm wel & $\begin{array}{l}\text { P. tremuloides } \\
\text { climax or sleble }\end{array}$ & $\begin{array}{l}\text { P. angalmennll } \\
\text { Populus balsamilara }\end{array}$ \\
\hline Cool dry & $\begin{array}{l}\text { P. Iramuloidas } \\
\text { serel to } \\
\text { A. leslocarpe } \\
\text { P. engelmennll }\end{array}$ & $\begin{array}{l}\text { A. leslocerpe } \\
\text { P. engalmannll }\end{array}$ \\
\hline Warm dry & $\begin{array}{l}\text { P. tramuloldes } \\
\text { serel to } \\
\text { P. menzles"l }\end{array}$ & P. menzles" \\
\hline
\end{tabular}

A. m/ser

Powell 1987

A. glabrum

B. repens

A. montlgenum

O. depauporero
T. tendlerl

Mountains of

soulheastern

Idaho, Ulah,

Nevada, western
Wyoming $(5,500$.

Wyoming $(5,500$
$8,800)$, and

$8,800)$, and.
central and

central and

$(8,000-8,500)$

Warm dry

S. orilcinum londierl C.T.

S. oreophllusliall forb C.T.

P. IramuloidasiA. alniliolla.

P. Iramuloldes/A. alnillolla-

[P. Iramuloldes/A.

[P. Iramulo/desia. alnillollo

tall forb C.T.]

Populus tremuloldas.

Ablas concolon

Arclostephylos palula C.T.

Mountalns of

eastern Nevad
$(8,300-8,500)$

Mountalns of

soulh.centra

Colorado:

Front Aanoe

Colorado

$(9,500 \cdot 10,500)$

Cool dry

P. tromulo/dos

Usuelly pure stands.

A. uva.ursl

S. oraophllus

Bromus portorl

C. 100 noe

Carex geophlla

P. Contorte P. contorte

A. laslocarpa
A. alnilolla

P. virginiane

S. oreophllus

Aster engelmann!l

o. chilansis

A. ainilollo

B. repens

P. virginiana

S. betullolle

Agropyron trechycaulum

Elymus glaucus

Elymus gla

P. tremuloldes cllmax (CO).

Stable or grazlng
dlscllmex (ID,NE,

UT,WY)

Usually pure stands

A. alnllolle

A. glabrum

P. myrsinites

P. virginiane

o. gamball

S. batulliolla

S. oreophllus

c. rubascens

B. carinelus

C. gayerl

A. engalmannll

Gerenium viscosissimum

O. chllans/s

P. equllinum
$T$. tendiert

$\begin{array}{ll}\text { P. tremuloldos } & \text { A. concolor } \\ \text { seral to } & \text { P. monzlesil } \\ \text { A. concolor } & \end{array}$

A. pelula

B. repans

P. Iendierian

C. rossil

Penstomon watsont

. Jameslana

Mueggler 1987

Mueggler 1987
Johnston and Hendzel 1985 Komarkova el al.

Mueggler 1987 



\begin{tabular}{|c|c|c|c|c|c|c|}
\hline $\begin{array}{l}\text { Habltat type or } \\
\text { communliy type }\end{array}$ & $\begin{array}{l}\text { Locatlon and } \\
\text { elevation (feet) }\end{array}$ & Silte & $\begin{array}{l}\text { Successlonal } \\
\text { status }\end{array}$ & $\begin{array}{c}\text { Tree } \\
\text { assoclatas }\end{array}$ & $\begin{array}{l}\text { Principel undergrowth } \\
\text { speclas }\end{array}$ & Authorlity \\
\hline $\begin{array}{l}\text { Populus Iramuloides/ } \\
\text { Artamisla Iridentata C.T. }\end{array}$ & $\begin{array}{l}\text { Mountains of } \\
\text { southaastern } \\
\text { Idaho, Ulah, } \\
\text { Nevada, and } \\
\text { westarn Wyoming } \\
(8,900 \cdot 9,400)\end{array}$ & Warm dry & $\begin{array}{l}\text { P. tremuloidas } \\
\text { stabla or seral } \\
\text { to unknown } \\
\text { ullimate cllmax }\end{array}$ & Usually pure stands & $\begin{array}{l}\text { A. tridentata } \\
\text { J. communis } \\
\text { S. oraophllus } \\
\text { B. clllatus } \\
\text { S. occldantalls } \\
\text { raraxicum olficinale }\end{array}$ & Mueggler 1987 \\
\hline $\begin{array}{l}\text { Populus tremuloldest } \\
\text { Barberis rapens H.T. }\end{array}$ & $\begin{array}{l}\text { Mountains of } \\
\text { southwaslern } \\
\text { North Dakola, and } \\
\text { southeastern } \\
\text { Monlana } \\
(3,500-4,000)\end{array}$ & $\begin{array}{l}\text { Warm dry } \\
\text { to wall. } \\
\text { drainad }\end{array}$ & $\begin{array}{l}\text { P. tremuloldas } \\
\text { cllmax }\end{array}$ & $\begin{array}{l}\text { Usually pure slands. } \\
\text { May contain } \\
\text { A. nagundo } \\
\text { F. pannsyivanica }\end{array}$ & $\begin{array}{l}\text { B. rapans } \\
\text { A. glabrum } \\
\text { P. virginlana } \\
\text { S. albus } \\
\text { P. pralans/s } \\
\text { G. boraala }\end{array}$ & $\begin{array}{l}\text { Hansen and } \\
\text { Hollman } 1988\end{array}$ \\
\hline $\begin{array}{l}\text { Populus tremuloldes/ } \\
\text { Betula occldenlalls H.T. }\end{array}$ & $\begin{array}{l}\text { Theodore Roosevall } \\
\text { Natlonal Perk, } \\
\text { North Dakota } \\
(2,400-2,800)\end{array}$ & $\begin{array}{l}\text { Warm well. } \\
\text { dralned }\end{array}$ & $\begin{array}{l}\text { P. Iramuloldes } \\
\text { cllmax }\end{array}$ & $\begin{array}{l}\text { J. scopulorum } \\
\text { A. negundo } \\
\text { Crataagus chrysocarpa } \\
\text { F. pannsylvanica } \\
\text { Uimus amaricana }\end{array}$ & $\begin{array}{l}\text { B. occldantalls } \\
\text { P. virginiana } \\
\text { Symphoricarpos spp. } \\
\text { roxicodandron rydbergll } \\
\text { D. micrantha } \\
\text { Apocynum androsaemilollum }\end{array}$ & $\begin{array}{l}\text { Hansen et al. } \\
1984\end{array}$ \\
\hline $\begin{array}{l}\text { Populus lremuloldes. } \\
\text { Corylus cornula H.T.(SD,WY); } \\
\text { C.T.(CO) } \\
\text { Aralla nudicaulls phase (SD) } \\
\text { Pteridium aquillnum phasa (SD) }\end{array}$ & $\begin{array}{l}\text { Elack Hills and } \\
\text { Bear Lodge } \\
\text { Mountains, South } \\
\text { Dakota and } \\
\text { eastern Wyoming } \\
(3,900-6,300)_{i} \\
\text { Front Ranga, } \\
\text { Colorado }(\geq 8,000)\end{array}$ & $\begin{array}{l}\text { Warm molst } \\
10 \text { well- } \\
\text { dratned }\end{array}$ & $\begin{array}{l}\text { P. Iremuloldas } \\
\text { cllmax (SD,WY). } \\
\text { Slable or seral } \\
\text { (CO) 10 } \\
\text { P. manzias/l } \\
\text { P. pungens }\end{array}$ & $\begin{array}{l}\text { P. manzlas } /(\mathrm{CO}) \\
\text { P. pungens (CD) } \\
\text { Beluta papyrifara }\end{array}$ & $\begin{array}{l}\text { C. cornuta } \\
\text { S. arbus } \\
\text { A. nudicaulls } \\
\text { Astar clllolatus } \\
\text { G. Irillorum } \\
\text { O. chllans/s } \\
\text { P. aquillnum } \\
\text { V. canadansis }\end{array}$ & $\begin{array}{l}\text { Hoffman and } \\
\text { Alexander } 1987 \\
\text { Powell } 1987\end{array}$ \\
\hline $\begin{array}{l}\text { Populus tremuloldes- } \\
\text { Ables laslocarpal } \\
\text { Juniperus communis C.T. }\end{array}$ & $\begin{array}{l}\text { Mountains ol } \\
\text { northern Ulah and } \\
\text { eastern Nevada } \\
(8,000-10,000)\end{array}$ & Cool dry & $\begin{array}{l}\text { P. Iramuloldes } \\
\text { saral to } \\
\text { A. laslocarpa } \\
\text { P. engalmannil }\end{array}$ & $\begin{array}{l}\text { A. lesiocarpa } \\
\text { P. engalmannll }\end{array}$ & $\begin{array}{l}\text { J. communis } \\
\text { B. repens } \\
\text { S. oreophllus } \\
\text { Bromus spp. } \\
\text { S. occldentalls } \\
\text { A. millafollum } \\
\text { F. vesca } \\
\text { (F. americana) } \\
\text { r. oflicinale }\end{array}$ & Mueggler 1987 \\
\hline $\begin{array}{l}\text { Oopulus tremuloldas. } \\
\text { Pseudolsuga menzlasill } \\
\text { Juniperus communis C.T. }\end{array}$ & $\begin{array}{l}\text { Mounlains of } \\
\text { northern Ulah. } \\
\text { plateaus of } \\
\text { southarn Utah, } \\
\text { and mountalns ol } \\
\text { eastern Nevada } \\
(7,500-9,200)\end{array}$ & Warm dry & $\begin{array}{l}\text { P. lremuloldes } \\
\text { seral to } \\
\text { P. menziesil }\end{array}$ & P. menzlesil & $\begin{array}{l}\text { J. communis } \\
\text { B. repens } \\
\text { S. oreophilus } \\
\text { A. trachycaulum } \\
\text { S. occldantalls } \\
\text { Antennarla microphylla } \\
\text { A. miser } \\
\text { T. fendlert }\end{array}$ & Mueggler 1987 \\
\hline $\begin{array}{l}\text { Populus tremuloldes- } \\
\text { PInus contortal } \\
\text { Juniperus communis C.T. }\end{array}$ & $\begin{array}{l}\text { Mountalns ol } \\
\text { northern Utah } \\
(\geq 8,000)\end{array}$ & Cool dry & $\begin{array}{l}\text { P. lremuloldes } \\
\text { seral to unknown } \\
\text { ultimate cllmax. } \\
\text { Probably } \\
\text { A. laslocarpa } \\
\text { P. manzlesil }\end{array}$ & $\begin{array}{l}\text { A. laslocarpa } \\
\text { P. manzlesil } \\
\text { P. angalmannil } \\
\text { P. contorta }\end{array}$ & $\begin{array}{l}\text { J. communis } \\
\text { B. rapans } \\
\text { S. oraophlius } \\
\text { A. trachycaulum } \\
\text { C. geyerl } \\
\text { A. millafollum } \\
\text { A. miser } \\
\text { L. argenleus }\end{array}$ & Mueggler 1987 \\
\hline
\end{tabular}

Populus tremuloldas! Juniparus comm
C.T.(CO,UT.Wr)

[P. Iremuloldes/J. communis.

Carex geyerl C.T.(UT)

IP. tremuloldes/. communis.

Astragalus misar C.T.(UT)]
[P. Iremuloides/S. communls.

[P. Iremuloldes/J. communis-
Luplnus argenieus C.T.(UT,WY) $]$

Populus Iremuloides!

Lonicera involucrata C.T.

Front Range, central Colorado $(9,700-10,200)$

Mountains of wastern Wyoming, and Ulah $(8,000$ ,000); Front

Range, central $9,000-9,500)$

$(9,700-10,200)$

Front Range,

Populus tremuloldesl

Physocarpus monogynus C.T.

centrat Colorado

$(8,500 \cdot 9,500)$

Populus tremuloidas!

(Riparlan foresi)

Mounlalns of

south central

$(9,760 \cdot 10,600)$

Populus tramuloidest
Rubus parvilliorus H.T.(CD)

Mountalns of western Wyomin is 300$)$, western Colorado (8,000-10,000)

Mountalns of soulheastern Idaho aastarn Neva
$(5,800 \cdot 7,400)$

Populus Iramuloidest Sallx scoulerlana C. outhaastern Idaho northern Utah, and
Cool dry

P. Iramuloldes
climax, stable.
or seral 10
unknown ullimala
climax.
Probably
P. menziesil

Cool molst to well-
drained

Cool mols

P. tramuloldes
saral to saral to P. menzlesit

Cool mols

P. tremuloides sarat 10

$P$. angalmann

Cool molst

P. Hemuloldes Stable or saral ullimale cllmax (ID,UT,WY). Probably A. lasiocarpa Warm to P. tremuloldes well. dralned P. tremuloldes
stable. May be climax

Usually pura stands

$$
\text { Usually pura stands }
$$
P. manzlesil A. laslocarpa $(\mathrm{CO})$
$P$. engelmann
P. $P$. pungans (CD) P. contort
$P$. llexills

A. laslocarpa P. balsamiler

P. menzlasil engalmannil

P. angelmannil

Usually pure slands. May contain A. lasiocarpa P. menziasil (ID,UT,WY)

ainifolla

P. virginiana

B. carinalus

E. glaucus

O. chllansis

S. racemosa

Sambucus ceurl

A. Irachycaulum

B. carinalus

Martansia arizonica

D. occidantalls

Rudbackla occldantalls

T. landierl

S. canadans/s

B. repans

R. Woods

E. glaucus

O. chilensis

r. tendiarl
Mauk and Henderson 198 Mueggler 198

Powell 1987

Powell 1987 Radloff 1983

Powell 1987

Powell 198

Powell 1987

Hollman 1988 Muaggler 1987

\section{Muag} western Wyoming, and norlhern Utah and norlhern
$(7,000,8,300)$

$\begin{array}{lll}\text { Cool dry } & \text { P. tramuloldes } & \text { A. laslocarpa } \\ \text { to well. } & \text { seral lo } & \text { P. engeimannll } \\ \text { dralned } & \text { A. lastocarpa } & \text { P. contorta } \\ & \text { P. engalmannil } & \text { P. Hexills }\end{array}$





\begin{tabular}{|c|c|c|c|c|c|c|}
\hline $\begin{array}{l}\text { Habltat typ } s \text { or } \\
\text { community typ } s\end{array}$ & $\begin{array}{l}\text { Location and } \\
\text { elavatlon (IaBt) }\end{array}$ & SIta & $\begin{array}{l}\text { Successlonal } \\
\text { status }\end{array}$ & $\begin{array}{c}\text { Tras } \\
\text { assoclates }\end{array}$ & $\begin{array}{l}\text { Principal undargrowth } \\
\text { spoclas }\end{array}$ & Authority \\
\hline $\begin{array}{l}\text { Populus tremuloldes/ } \\
\text { Shepherdla canadensis C.T. }\end{array}$ & $\begin{array}{l}\text { Mountains of } \\
\text { soulheaslern Idaho } \\
\text { and wastern Wyoming } \\
(8,000 \cdot 9,000) \text {; } \\
\text { Front Range, } \\
\text { central Colorado } \\
(9,000 \cdot 10,000)\end{array}$ & $\begin{array}{l}\text { Cool dry } \\
\text { to well. } \\
\text { dralned }\end{array}$ & $\begin{array}{l}\text { P. Iremuio/des } \\
\text { soral to unknown } \\
\text { ultimate cllmax. } \\
\text { Probably } \\
\text { A. laslocarpa } \\
\text { p. engeimannll } \\
\text { P. menzlesill }\end{array}$ & $\begin{array}{l}\text { A. laslocarpa } \\
\text { P. engelmannll } \\
\text { P. menzlesh } \\
\text { P. contorla } \\
\text { p. flexills }\end{array}$ & $\begin{array}{l}\text { S. canadansis } \\
\text { B. repans } \\
\text { J. communis } \\
\text { R. woods/l } \\
\text { B. clllatus } \\
\text { G. boreale } \\
\text { G. viscosissimum } \\
\text { L. argentaus } \\
\text { O. chllensis }\end{array}$ & $\begin{array}{l}\text { Powell } 1987 \\
\text { Musggler } 1987\end{array}$ \\
\hline 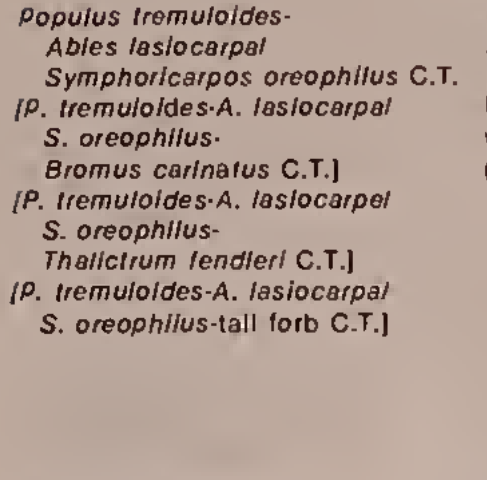 & $\begin{array}{l}\text { Mountains of } \\
\text { southeastern } \\
\text { Idaho, northern } \\
\text { Ulah, and } \\
\text { wastern Wyoming } \\
(7,000-9,000)\end{array}$ & $\begin{array}{l}\text { Warm dry } \\
\text { to wall- } \\
\text { dralned }\end{array}$ & $\begin{array}{l}\text { P. Iremuloldes } \\
\text { soral to unknown } \\
\text { ullimate cllmax. } \\
\text { Probably } \\
\text { A. laslocarpa }\end{array}$ & $\begin{array}{l}\text { May be pure stands. } \\
\text { Usually conlain } \\
\text { A. laslocarpo } \\
\text { A. concolor } \\
\text { p. menzies } /\end{array}$ & $\begin{array}{l}\text { S. oreophilus } \\
\text { A. ainilolla } \\
\text { A. tridentale } \\
\text { B. repens } \\
\text { P. virginlana } \\
\text { B. cerlnatus } \\
\text { C. rubescens } \\
\text { E. glaucus } \\
\text { P. pratensis } \\
\text { C. geyeri } \\
\text { G. viscosissimum } \\
\text { L. argenteus } \\
\text { R. occlientalls } \\
\text { Senecio serre } \\
\text { T. fendien }\end{array}$ & $\begin{array}{l}\text { Muagglar } 1987 \\
\text { Staala at al. } 1983\end{array}$ \\
\hline $\begin{array}{l}\text { Populus Iremuloldes- } \\
\text { Ables concolorl } \\
\text { Symphoricerpos oreophlius C.T. }\end{array}$ & $\begin{array}{l}\text { Mountatns ol } \\
\text { northern Ulah and } \\
\text { aastern Navada } \\
(7,000-9,000)\end{array}$ & Warm dry & $\begin{array}{l}\text { P. Iremuloides } \\
\text { seral to } \\
\text { A. concolor }\end{array}$ & $\begin{array}{l}\text { A. concolor } \\
\text { p. pungens }\end{array}$ & $\begin{array}{l}\text { S. oreophllus } \\
\text { B. repens } \\
\text { A. woodsll } \\
\text { A. trachycaulum } \\
\text { E. glaucus } \\
\text { A. engelmannll } \\
\text { R. occldentalls } \\
\text { O. chllensis } \\
\text { S. serra }\end{array}$ & Musggler 1987 \\
\hline $\begin{array}{l}\text { Populus tremuloldes- } \\
\text { Pseudolsuga menziesill } \\
\text { Sym phoricarpos oreophilius C.T. }\end{array}$ & $\begin{array}{l}\text { Mounlalns of } \\
\text { southeastarn } \\
\text { Idaho and } \\
\text { northern Utah } \\
(6,000 \cdot 7.500)\end{array}$ & Warm dry & $\begin{array}{l}\text { P. Iremuloldes } \\
\text { seral to } \\
\text { P. menzies/l }\end{array}$ & $\begin{array}{l}\text { P. menzlesil } \\
\text { A. laslocarpa } \\
\text { P. contorla }\end{array}$ & $\begin{array}{l}\text { S. oreophllus } \\
\text { R. woodsll } \\
\text { C. rubescens } \\
\text { E. glaucus } \\
\text { G. viscos/ssimum } \\
\text { L. argenteus } \\
\text { T. landleri }\end{array}$ & Mueggler 1987 \\
\hline $\begin{array}{l}\text { Populus tremuloldes- } \\
\text { Plnus contortal } \\
\text { Symphoricarpos oreophilus C.T. }\end{array}$ & $\begin{array}{l}\text { Mountains of } \\
\text { southeastern } \\
\text { Idaho and } \\
\text { northern Idaho } \\
(5,700-9,800)\end{array}$ & Warm dry & $\begin{array}{l}\text { P. tremuloides } \\
\text { seral to unknown } \\
\text { ulltmale cllmax }\end{array}$ & P. contorta & $\begin{array}{l}\text { S. oreophllus } \\
\text { P. myrsinites } \\
\text { C. rubescens } \\
\text { C. geyerl } \\
\text { G. viscosissimum } \\
\text { o. chllensis } \\
\text { T. fendlent }\end{array}$ & Mueggler 1987 \\
\hline $\begin{array}{l}\text { Populus iremuloldest } \\
\text { Symphoricarpos oreophlius H.T., } \\
\text { C.T.(CO);C.T.(ID.UT.NE,WY) } \\
\text { (P. tremuloldes/S. oreophilus- } \\
\text { Wyerhis amplexicaulls C.T.1 }\end{array}$ & $\begin{array}{l}\text { Mountalns of } \\
\text { Ulah, south. } \\
\text { eastern Idaho, } \\
\text { northwestern } \\
\text { Wyoming, eastern }\end{array}$ & $\begin{array}{l}\text { Warm molst } \\
\text { to well. } \\
\text { drained }\end{array}$ & $\begin{array}{l}\text { P. tremuloldes } \\
\text { climax (CO). } \\
\text { Slable or seral } \\
\text { to unknown } \\
\text { ullimate cllmax }\end{array}$ & $\begin{array}{l}\text { Usually pure stands. } \\
\text { Seral stands in } \mathrm{CO} \\
\text { may contatn } \\
\text { A. laslocarpa } \\
\text { P. menzlestl }\end{array}$ & $\begin{array}{l}\text { S. oreophllus } \\
\text { B. repens } \\
\text { o. myrstnlies } \\
\text { o. gambelli } \\
\text { W. amplexlcaulls }\end{array}$ & $\begin{array}{l}\text { Hess and Wasser } 1982 \\
\text { Hoftman } 1988 \\
\text { Hoflman and } \\
\text { Atexander } \\
1980.1983\end{array}$ \\
\hline 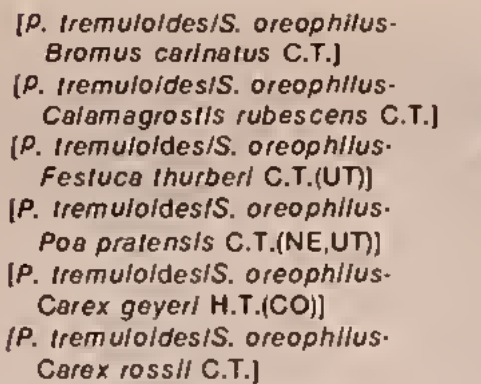 & $\begin{array}{l}\text { Nevada }(7,000 \\
9,000) \text {, and } \\
\text { centrat and } \\
\text { western Colorado } \\
(8,000 \cdot 10,000)\end{array}$ & & $\begin{array}{l}\text { (CO,UT,ID,WY). } \\
\text { Probably } \\
\text { A. laslocarpa } \\
\text { p. menzlesil }\end{array}$ & $\begin{array}{l}\text { P. engelmannil } \\
\text { P. contorta } \\
\text { P. ponderosa }\end{array}$ & $\begin{array}{l}\text { 8. carlnatus } \\
\text { C. rubescens } \\
\text { F. thurberi } \\
\text { P. pratensis } \\
\text { C. geyell } \\
\text { C. rossil } \\
\text { A. engelmannll } \\
\text { M. arizonica } \\
\text { R. occidentells } \\
\text { S. sarra } \\
\text { T. fendlerl }\end{array}$ & $\begin{array}{l}\text { Johnston and } \\
\text { Handzal 1985 } \\
\text { Komarkova et at. } \\
1988 \\
\text { Mauk and } \\
\text { Henderson } 1984 \\
\text { Mueggler } 1987 \\
\text { Powell } 1987\end{array}$ \\
\hline
\end{tabular}

P. Iremuloldes/S. oreophllus.

Thallctrum lendlerl C.T.J

[P. Iremulo/desis. oreophllus. lall forb C.T.]

Populus Iremuloides!

Vaccinium caespilosum C.T.

Front Range, ceniral Colorado cenlral Colo
$(7,600 \cdot 7,800)$

Front Range, central Colorado $(9,000 \cdot 10,500)$

Populus tremuioldesi Vaccinlum myrilius C.T.

Populus tremulodies!

Wyethia amplexicaulis C.T.

soulheastern

Idaho, Utah,

eastern Novada,

and wastern

Mountatns of

southeaslern

Idaho, Ulah,

easlern Nevad
and western

Wyoming

$(6,200-10,000)$

Populus Iremuloldes/

Fronl Range, central Colorado
$(9,000 \cdot 10,200)$

Bromus clllatus C.T.

Fronl Range,

Populus tremuloldest $(9,000 \cdot 10,500)$

Popuius tromuloides. Careudolsuga monzlesill

Mountains of southaaster
idaho and Western Wyoming
Cool molsl P. tramuloldes P. menzlesil Io wall.

P. tramulor

P. menzies

Cool dry

P. tremuloides seral to A, laslocarpa A. concolor
P. engelmannil

Cool wall. dralned

P. Iremuloldes slable or seral to unknown uitimate cllmax

Warm to
cool dry

p. tremulo/des slable or seral to unknown

ultimate cllmax. May be grazlng disclimax

Cool molst

P. tremulodies seral to

A. concolor

A. laslocarpe

Cool molsi

P. tromuloldes

seral to

A. lasiocarpa

P. engelmannl

Cool dry

P. tremuloldes seral lo Peral menziosh

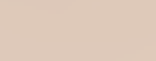

A. teslocarpa A. concolor
$P$. engeimannll

Usually pura stands

Usuatly pure stands

the

A. concolor

A. loslocarpa

P. engelmann

p. pungens

P. contorla

A. laslocarpa

P. ongelmannl

A. concolor

P. contorta

P. menzlesil
p. contorta
V. caespllosum

A. glabrum

A. ainlfolla

R. woods/
E. glaucus

A. cordllolla

V. myrtilius

B. repens

o. monogynus

V. scoparium
A. cordifolla

W. amplexicaulls

8. carlnatus
p. protensis

P. pratensis
G. viscosissimum

R. occidentalls

S. sorra

8. cerinalus

A. Irachycaulu

E. glaucus
P. pratens/s

P. pratensis

A. occidentells

A. occidentells
T. Iend/arl

v. americana

B. clllatus

A. uva.ursl

J. communls

Poa spp.

Poa spp.

S. stellata

C. canedensis

R. woodsII

P. pratensis

Ligusticum portor

S. triangularls

c. rubescons

S. oreophilu

E. gleucus

C. geyerl

A. cordifolla

L. argentous

o. chilens/s

r. fendierl
Powell 1987

Powell 1987

Mueggler 1987

Muagglar 1987

Powell 1987

Powall 1987 Radloff 1983 



\begin{tabular}{|c|c|c|c|c|c|c|}
\hline $\begin{array}{l}\text { Hebltel type or } \\
\text { communlty type }\end{array}$ & $\begin{array}{l}\text { Locetlon and } \\
\text { elevetton (leet) }\end{array}$ & Slie & $\begin{array}{l}\text { Successlonal } \\
\text { stetus }\end{array}$ & $\begin{array}{c}\text { Tree } \\
\text { essocletes }\end{array}$ & $\begin{array}{l}\text { Princlpal undergrowth } \\
\text { specles }\end{array}$ & Authorlity \\
\hline $\begin{array}{l}\text { populus tremuloidest } \\
\text { Calamagrostls rubescens H.T. } \\
\text { (SE WY);C.T.(NW WY,ID,UT) }\end{array}$ & $\begin{array}{l}\text { Mounteins of } \\
\text { southesstern } \\
\text { Ideho, Uteh, and } \\
\text { northwestern } \\
\text { end southesstern } \\
\text { Wyoming } \\
(8,000 \cdot 8,600)\end{array}$ & Cool dry & $\begin{array}{l}\text { P. Iremuioldes } \\
\text { cllmex (SE WY). } \\
\text { Stoble or seral } \\
\text { to unknown } \\
\text { ultimete cllmax } \\
\text { (NWW WY,ID,UT). } \\
\text { Probebly } \\
\text { A. /esiocarpa } \\
\text { P. menzlesll }\end{array}$ & $\begin{array}{l}\text { Usually pure stands } \\
\text { (SE WY). } \\
\text { Mey conteln } \\
\text { A. lesiocarps } \\
\text { p. menzlesll } \\
\text { P. contorts } \\
\text { (NW WY,ID,UT) }\end{array}$ & $\begin{array}{l}\text { C. rubescens } \\
\text { S. oreophllus } \\
\text { o. pratensis } \\
\text { C. geyerl } \\
\text { Fragarla spp. } \\
\text { G. viscosissimum } \\
\text { O. chllensis } \\
\text { T. Iendierl }\end{array}$ & $\begin{array}{l}\text { Alexender el al. } \\
1986 \\
\text { Mueggler } 1987\end{array}$ \\
\hline $\begin{array}{l}\text { Populus tremuloldesi } \\
\text { Elymus glaucus C.T. }\end{array}$ & $\begin{array}{l}\text { Front Renge, } \\
\text { centrel Coloredo } \\
(9,500 \cdot 10,000)\end{array}$ & Cool wet & $\begin{array}{l}\text { P. tremuloldes } \\
\text { seral to } \\
\text { A. toslocarps } \\
\text { p. engelmannll }\end{array}$ & $\begin{array}{l}\text { A. laslocarpa } \\
\text { p. engeimannll }\end{array}$ & $\begin{array}{l}\text { E. glaucus } \\
\text { B. cllialus } \\
\text { o. pratensis } \\
\text { Aconlium columblenum } \\
\text { Erlgeron spp. } \\
\text { Heracleum sphondyllum } \\
\text { (H. lanatum) }\end{array}$ & Powell 1987 \\
\hline $\begin{array}{l}\text { Populus tremuloidesi } \\
\text { Fesiuca arlzonica H.T. }\end{array}$ & $\begin{array}{l}\text { Mountains ol } \\
\text { south.centrat } \\
\text { Colorado } \\
(9,500 \cdot 10,000)\end{array}$ & Warm dry & $\begin{array}{l}\text { P. tremuloldes } \\
\text { cllmax }\end{array}$ & Usuelly pure stands & $\begin{array}{l}\text { F. arizonica } \\
\text { F. thurberl } \\
\text { M. montana } \\
\text { L. argenteus }\end{array}$ & $\begin{array}{l}\text { Komarkova et al. } \\
1988\end{array}$ \\
\hline $\begin{array}{l}\text { Populus tremuloldes/ } \\
\text { Festuca thurberl H.T.C.T.(CO); } \\
\text { C.T.(UT) } \\
\text { [P. IremuloldestF. thurberi- } \\
\text { Carex geyerl H.T.(CO)] }\end{array}$ & $\begin{array}{l}\text { Mountelns of } \\
\text { Uteh }(8,000 . \\
10.000) \text { and } \\
\text { Colorado } \\
(9,000-10,500)\end{array}$ & Werm dry & $\begin{array}{l}\text { P. tremuloldes } \\
\text { cllmax or stable } \\
\text { (CO). Stable or } \\
\text { seral to unknown } \\
\text { ulimate climax } \\
\text { (UT). Probably } \\
\text { A. laslocarps } \\
\text { P. engelmannil }\end{array}$ & $\begin{array}{l}\text { Usuelly pure stands. } \\
\text { May contaln } \\
\text { A. laslocarpa } \\
\text { p. engelmannll } \\
\text { p. menzles } \\
\text { p. contorta } \\
\text { p. llexills }\end{array}$ & $\begin{array}{l}\text { F. thurberl } \\
\text { S. oreophllus } \\
\text { B. carinatus } \\
\text { S. occldentalls } \\
\text { C. geyen } \\
\text { L. leucenthus } \\
\text { T. ollicinale } \\
\text { T. lendient }\end{array}$ & $\begin{array}{l}\text { Hess and } \\
\text { Alexander } 1986 \\
\text { Hess and Wasser } \\
1982 \\
\text { Johnston and } \\
\text { Hendzel } 1985 \\
\text { Komarkova el al. } \\
\text { 1988 } \\
\text { Mueggler } 1987 \\
\text { Powell 1987 }\end{array}$ \\
\hline $\begin{array}{l}\text { Populus tremuloides. } \\
\text { Ables concolorl } \\
\rho_{\text {os pratensis C.T. }}\end{array}$ & $\begin{array}{l}\text { Mounteins of } \\
\text { southern Uteh and } \\
\text { eastern Nevada } \\
(8,000-8,800)\end{array}$ & $\begin{array}{l}\text { Warm dry } \\
\text { to well. } \\
\text { dralned }\end{array}$ & $\begin{array}{l}\text { D. tremuloldes } \\
\text { seral to } \\
\text { A. concolor }\end{array}$ & $\begin{array}{l}\text { A. concolor } \\
\text { p. menziesil }\end{array}$ & $\begin{array}{l}\text { P. pratensis } \\
\text { J. communis } \\
\text { A. trachycaulum } \\
\text { B. carinalus } \\
\text { S. occidentelis } \\
\text { T. ollicinate } \\
\text { T. lendlerl }\end{array}$ & Mueggler 1987 \\
\hline $\begin{array}{l}\rho_{\text {Opulus Iremuioldesl }} \\
\rho_{O Q} \text { pratensis C.T. }\end{array}$ & $\begin{array}{l}\text { Mountains ol } \\
\text { southeastern } \\
\text { Ideho, southern } \\
\text { Uteh, and eastern } \\
\text { Nevada }(7,000 \text { - } \\
9,000) \text {; Front } \\
\text { Range, central } \\
\text { Colorado } \\
(8,500-9,600)\end{array}$ & $\begin{array}{l}\text { Cool molst } \\
\text { to well. } \\
\text { dralned }\end{array}$ & $\begin{array}{l}\text { P. tremuloldes } \\
\text { stable or seral } \\
\text { to unknown } \\
\text { ultimate climax } \\
\text { (ID,NE,UT). May be } \\
\text { grazing disclimax. } \\
\text { Serat (CO) to } \\
\text { A. lesiocarpe } \\
\text { A. concolor } \\
\text { P. pungens } \\
\text { P. menziesil }\end{array}$ & $\begin{array}{l}\text { A. lasiocarpa } \\
\text { A. concolor } \\
\text { p. pungens } \\
\text { p. menzles"l } \\
\text { p. engelmannll } \\
\text { p. ponderose } \\
\text { p. "lexills } \\
\end{array}$ & $\begin{array}{l}\text { P. pratensis } \\
\text { p. monogynus } \\
\text { B. carinatus } \\
\text { C. rubescens } \\
\text { p. nervosa } \\
\text { A. millelollum } \\
\text { S. stellera } \\
\text { T. ollicinale } \\
\text { T. lendlerl } \\
\text { Trllollum longlpes } \\
\text { V. americang }\end{array}$ & $\begin{array}{l}\text { Mueggler } 1987 \\
\text { Powell } 1987\end{array}$ \\
\hline $\begin{array}{l}\text { Populus tremuloidest } \\
\text { Stlpa comata C.T. }\end{array}$ & $\begin{array}{l}\text { Mountalns of } \\
\text { southeestern tdaho } \\
\text { south to the } \\
\text { plateaus of } \\
\text { southern Utah } \\
(6,500-9.500)\end{array}$ & Warm dry & $\begin{array}{l}\text { P. tremutoldes } \\
\text { siable or seral } \\
\text { to unknown } \\
\text { ultimate cllmax. } \\
\text { Probably } \\
\text { P. ponderosa }\end{array}$ & $\begin{array}{l}\text { Usually pure stands. } \\
\text { May contaln } \\
\text { p. ponderose }\end{array}$ & $\begin{array}{l}\text { S. comata } \\
\text { F. Idahoensis } \\
\text { P. fendlerlana } \\
\text { S. hystrix } \\
\text { A. microphylla } \\
\text { L. argenteus } \\
\text { T. olllelnale }\end{array}$ & Mueggler 1987 \\
\hline $\begin{array}{l}\text { Populus tremuloldes/ } \\
\text { Carex loeneo C.T. }\end{array}$ & $\begin{array}{l}\text { Front Range. } \\
\text { central Colorado } \\
(9,300 \cdot 10,700)\end{array}$ & Cool molst & $\begin{array}{l}\text { P. tremuloldes } \\
\text { seral lo } \\
\text { A. concolor } \\
\text { P. menziesh } \\
\text { (tow elevations) } \\
\text { A. laslocarpa } \\
\text { P. engelmannll } \\
\text { (high elevations) }\end{array}$ & $\begin{array}{l}\text { A. concolor } \\
\text { P. menzles"l } \\
\text { A. lastocarpa } \\
\text { P. engetmannill } \\
\text { P. contorta } \\
\text { P. llexills }\end{array}$ & $\begin{array}{l}\text { C. loenea } \\
\text { B. cllialus } \\
\text { A. woodsll } \\
\text { A. millelollum } \\
\text { A. miser } \\
\text { F. ovalls } \\
\text { (F. virginiana) } \\
\text { Thermopsis divaricarpa }\end{array}$ & Powell 1987 \\
\hline $\begin{array}{l}\text { Populus tremuloides. } \\
\text { Ables laslocarpal } \\
\text { Carex geverl C.T. }\end{array}$ & $\begin{array}{l}\text { Mountalns of } \\
\text { southeastern } \\
\text { Idaho, Uteh, } \\
\text { and western } \\
\text { Wyoming } \\
(6,000-10,000)\end{array}$ & Coot dry & $\begin{array}{l}\text { p. Iremuloides } \\
\text { seral to } \\
\text { A. laslocarpe } \\
\text { p. engelmennill }\end{array}$ & $\begin{array}{l}\text { A. laslocarpa } \\
\text { p. engeimannil } \\
\text { p. menzles ll }\end{array}$ & $\begin{array}{l}\text { C. geyerl } \\
\text { S. oreophlius } \\
\text { C. rubescens } \\
\text { S. occidentelis } \\
\text { C. rossil } \\
\text { A. millolollum } \\
\text { A. miser } \\
\text { F. vesca } \\
\text { (F. americena) } \\
\text { O. chllensis } \\
\text { T. lendlerl } \\
\text { T. longlpes }\end{array}$ & Mueggler 1987 \\
\hline $\begin{array}{l}\text { Populus tremuloides- } \\
\text { Plnus contorlal } \\
\text { Carex geyerl C.T. }\end{array}$ & $\begin{array}{l}\text { Mountains of } \\
\text { southeastern } \\
\text { Idaho and } \\
\text { northeastern Utah } \\
(8,200 \cdot 9,400)\end{array}$ & Cool dry & $\begin{array}{l}\text { P. tremuloides } \\
\text { seral to unknown } \\
\text { ultimate cllmax. } \\
\text { Probably } \\
\text { A. lasiocarps } \\
\text { P. menziesil }\end{array}$ & $\begin{array}{l}\text { A. laslocarpa } \\
\text { p. menzles/l } \\
\text { p. contorta }\end{array}$ & $\begin{array}{l}\text { C. geyerl } \\
\text { V. scoparium } \\
\text { C. rubescens } \\
\text { L. argenteus } \\
\text { O. chllens/s } \\
\text { T. lendierl }\end{array}$ & Mueggler 1987 \\
\hline $\begin{array}{l}\text { Populus tremuloidest } \\
\text { Carex geyerl H.T. }\end{array}$ & $\begin{array}{l}\text { Mountains of } \\
\text { Utah, south. } \\
\text { eastern Wyoming, } \\
\text { and north.central } \\
\text { and west.contral } \\
\text { Colorado } \\
(7,500 \cdot 10,000)\end{array}$ & $\begin{array}{l}\text { Cool dry } \\
\text { to well. } \\
\text { dralned }\end{array}$ & $\begin{array}{l}\text { P. tremuloides } \\
\text { climax (CO,WY). } \\
\text { Seral to unknown } \\
\text { ult mate cllmex } \\
\text { (UT) }\end{array}$ & $\begin{array}{l}\text { Usuelly pure stands. } \\
\text { May contaln (CO) } \\
\text { A. lasiocarpe } \\
\text { p. contorla } \\
\text { p. llexllis }\end{array}$ & $\begin{array}{l}\text { G. geyent } \\
\text { B. repens } \\
\text { J. communis } \\
\text { S. oreophllus } \\
\text { C. rossll } \\
\text { C. rubescens } \\
\text { A. cordilolla } \\
\text { L. leucanthus } \\
\text { Liguslicum spp. } \\
\text { O. depauperata }\end{array}$ & $\begin{array}{l}\text { Alexander et at. } \\
1986 \\
\text { Hess end } \\
\text { Alexander } 1986 \\
\text { Holfman and } \\
\text { Alexander } 1983 \\
\text { Johnston end } \\
\text { Hendzel 1985 } \\
\text { Mauk sand Henderson } \\
1984\end{array}$ \\
\hline $\begin{array}{l}\text { Populus tremuloides. } \\
\text { Ables laslocarpal } \\
\text { Carex rossil C.T. }\end{array}$ & $\begin{array}{l}\text { Mountains ol } \\
\text { southern Utah } \\
\text { and eastern } \\
\text { Nevada } \\
(8,000 \cdot 10,300)\end{array}$ & Cool dry & $\begin{array}{l}\text { P. tremuloides } \\
\text { seral to } \\
\text { A. leslocarps } \\
\text { p. engelmennll }\end{array}$ & $\begin{array}{l}\text { A. lasiocarpa } \\
\text { p. engelmannll }\end{array}$ & $\begin{array}{l}\text { C. rossll } \\
\text { Bromus anomalus } \\
\text { A. miser } \\
\text { L. argenteus } \\
\text { T. ollicinale } \\
\text { Trillollum spp. }\end{array}$ & Mueggler 1987 \\
\hline $\begin{array}{l}\text { Populus tremuloidest } \\
\text { Carex ressil C.T. }\end{array}$ & $\begin{array}{l}\text { Mountains of } \\
\text { southern Utah } \\
\text { and eastern } \\
\text { Nevada } \\
(8,000 \cdot 10,500)\end{array}$ & Coot dry & $\begin{array}{l}\text { p. tremuioides } \\
\text { stable or serel } \\
\text { to unknown } \\
\text { ultimate climex. } \\
\text { Probebly } \\
\text { A. laslocarpo } \\
\text { P. engelmannil }\end{array}$ & $\begin{array}{l}\text { A. laslocerpa } \\
\text { p. engelmannil } \\
\text { p. Hexills }\end{array}$ & $\begin{array}{l}\text { C. rossll } \\
\text { A. tridentata } \\
\text { S. oreophilus } \\
\text { A. trachyceulum } \\
\text { B. anomalus } \\
\text { P. tendieriane } \\
\text { S. occldentalls } \\
\text { T. ollicinale }\end{array}$ & Mueggler 1987 \\
\hline $\begin{array}{l}\text { opulus tremuioldesl } \\
\text { Astragalus miser C.T. }\end{array}$ & $\begin{array}{l}\text { Mountalns of } \\
\text { western Wyoming } \\
\text { and Utah }(7,500 \cdot \\
10,000) \text {, and } \\
\text { south.central } \\
\text { Colorado } \\
(10,000 \cdot 10.600)\end{array}$ & $\begin{array}{l}\text { Coof to } \\
\text { well. } \\
\text { dralned }\end{array}$ & $\begin{array}{l}\text { p. tremuioldes } \\
\text { stable or seral } \\
\text { to unknown } \\
\text { ultimate climex. } \\
\text { May be grazlng } \\
\text { dlscllmex (UT). }\end{array}$ & D. Hex $\| / / \mathrm{s}$ & $\begin{array}{l}\text { A. miser } \\
\text { C. geyerl } \\
\text { A. millelollum } \\
\text { G. viscosissimum } \\
\text { L. ergenteus } \\
\text { T. lendierl }\end{array}$ & $\begin{array}{l}\text { Mueggler } 1987 \\
\text { Powell } 1987\end{array}$ \\
\hline
\end{tabular}





\begin{tabular}{|c|c|c|c|c|c|c|}
\hline $\begin{array}{l}\text { Mebitet type or } \\
\text { community type }\end{array}$ & $\begin{array}{l}\text { Location and } \\
\text { elevetion (feet) }\end{array}$ & Slte & $\begin{array}{l}\text { Successionel } \\
\text { status }\end{array}$ & $\begin{array}{c}\text { Tree } \\
\text { essocletes }\end{array}$ & $\begin{array}{l}\text { Principel undergrowth } \\
\text { spscles }\end{array}$ & Authority \\
\hline $\begin{array}{l}\text { Populus iremuloldesl } \\
\text { Heracleum sphondyllium H.T., } \\
\text { C.T. } \\
\text { [P. Tremuloldesl } \\
\text { Herecleum lenalum H.T.] }\end{array}$ & $\begin{array}{l}\text { Mountelns of } \\
\text { south-centrel end } \\
\text { western Coloredo } \\
(8,500-9,900)\end{array}$ & $\begin{array}{l}\text { Werm molet } \\
\text { to wet }\end{array}$ & $\begin{array}{l}\text { P. Iremuloides } \\
\text { climax or steble }\end{array}$ & $\begin{array}{l}\text { Usuelly pure stands. } \\
\text { Mey contaln } \\
\text { P. belsamilere }\end{array}$ & $\begin{array}{l}\text { H. sphondyllium } \\
\text { (H. tanatum) } \\
\text { A. tenullolla } \\
\text { B. clliatus } \\
\text { E. glaucus } \\
\text { Pedicularls bracteosa } \\
\text { T. fendlerl }\end{array}$ & $\begin{array}{l}\text { Hess and Wasser } \\
1982 \\
\text { Hoffman } 1988 \\
\text { Hoflman and } \\
\text { Alexender } 1980 . \\
1983 \\
\text { Powell } 1987\end{array}$ \\
\hline $\begin{array}{l}\text { Populus tremuloldest } \\
\text { Lathyrus teucanthus C.T. }\end{array}$ & $\begin{array}{l}\text { Front Range, } \\
\text { centrel Colorado } \\
(10,000-10,500)\end{array}$ & $\begin{array}{l}\text { Cool molst } \\
\text { to well. } \\
\text { drained }\end{array}$ & $\begin{array}{l}\text { P. Iremuioldes } \\
\text { stable or serel } \\
\text { to unknown } \\
\text { ullimete cllmex. } \\
\text { Mey be grazing } \\
\text { disclimax. }\end{array}$ & Usually pure stands & $\begin{array}{l}\text { L. levcanthus } \\
\text { A. woods/l } \\
\text { B. porlerl } \\
\text { F. thurberl } \\
\text { A. millelollum }\end{array}$ & Powell 1987 \\
\hline $\begin{array}{l}\text { Populus tremuloldes/ } \\
\text { Liguiaria bigelovil C.T. } \\
\text { [P. Iremuloldes } \\
\text { Seneclo bigelovil C.T.] }\end{array}$ & $\begin{array}{l}\text { Front Range, } \\
\text { central Colorado } \\
(8,500 \cdot 9,000)\end{array}$ & Cool moist & $\begin{array}{l}\text { P. tremuloldes } \\
\text { seral to unknown } \\
\text { ultimate cllmex. } \\
\text { Probably } \\
\text { P. menzles/l } \\
\text { P. pungens } \\
\text { A. concolor }\end{array}$ & $\begin{array}{l}\text { P. menzies } \\
\text { P. pungens } \\
\text { A. concolor } \\
\text { P. contorta }\end{array}$ & $\begin{array}{l}\text { L. blgelovil } \\
\text { J. communis } \\
\text { A. woodsil } \\
\text { F. ovalls } \\
\text { (F. virginlana) } \\
\text { G. boreale } \\
\text { G. richardsonll }\end{array}$ & Powell 1987 \\
\hline $\begin{array}{l}\text { Populus iremuloldesl } \\
\text { Ligusilicum porterl H.T.;C.T. }\end{array}$ & $\begin{array}{l}\text { Front Range, } \\
\text { central Colorado, } \\
\text { end mounlelns of } \\
\text { southwestern } \\
\text { Colorado } \\
(9,000-10,000)\end{array}$ & $\begin{array}{l}\text { Warm molst } \\
\text { to well. } \\
\text { dralned }\end{array}$ & $\begin{array}{l}\text { P. Iremuloldes } \\
\text { cllmex or seral } \\
\text { to unknown } \\
\text { ultimate cllmax, } \\
\text { Probably } \\
\text { A. concolor } \\
\text { P. pungens } \\
\text { P. menziesll }\end{array}$ & $\begin{array}{l}\text { Usually pure stands. } \\
\text { May contaln } \\
\text { A. concolor } \\
\text { P. pungens } \\
\text { P. menzlesll } \\
\text { P. Hexllis }\end{array}$ & $\begin{array}{l}\text { L. porterl } \\
\text { S. oreophlius } \\
\text { C. geyerl } \\
\text { L. leucanthus } \\
\text { T. lendlerl } \\
\text { V. americana }\end{array}$ & $\begin{array}{l}\text { Johnston and } \\
\text { Hendzel } 1985 \\
\text { Powell } 1987\end{array}$ \\
\hline $\begin{array}{l}\text { Populus Iremuloidesl } \\
\text { Luplnus argenteus H.T. }\end{array}$ & $\begin{array}{l}\text { Bighorn Mountains, } \\
\text { north-central } \\
\text { Wyoming } \\
(7,000 \cdot 7,800)\end{array}$ & $\begin{array}{l}\text { Werm well- } \\
\text { dralned }\end{array}$ & $\begin{array}{l}\text { P. Iremuloldes } \\
\text { cllmex }\end{array}$ & Usually pure stands & $\begin{array}{l}\text { Lupinus spp. } \\
\text { Carex spp. } \\
\text { Trilollum spp. }\end{array}$ & $\begin{array}{l}\text { Hoflman and } \\
\text { Alexander } 1976\end{array}$ \\
\hline $\begin{array}{l}\text { Populus tremuloldesl } \\
\text { Preridium aquillinum H.T.C.T. } \\
\text { (CO);C.T.(UT) }\end{array}$ & $\begin{array}{l}\text { Front Range, } \\
\text { central Colorado; } \\
\text { mountalns of } \\
\text { western and } \\
\text { south-central } \\
\text { Coloredo } \\
(8,000 \cdot 9,000) \text {, } \\
\text { and norhern Uteh } \\
(5,800 \cdot 9.400)\end{array}$ & $\begin{array}{l}\text { Werm } \\
\text { poorly. } \\
\text { dralned }\end{array}$ & $\begin{array}{l}\text { P. Iremulo/des } \\
\text { climax or stable } \\
\text { (CO). Slable or } \\
\text { serel to unknown } \\
\text { ullimate cllmax } \\
\text { (UT) }\end{array}$ & $\begin{array}{l}\text { Usually pure slands. } \\
\text { Moy contain } \\
\text { P. menzlesh (FR CO) }\end{array}$ & $\begin{array}{l}\text { P. equilinum } \\
\text { B. carinatus } \\
\text { E. gleucus } \\
\text { C. geyerl } \\
\text { Melica subuleta } \\
\text { R. occidentells } \\
\text { S. serra } \\
\text { T. lendierl }\end{array}$ & $\begin{array}{l}\text { Hoffman } 1988 \\
\text { Hoflman and } \\
\text { Alexander } 1980 . \\
\text { 1983 } \\
\text { Komarkova el al. } \\
\quad 1988 \\
\text { Mueggler } 1987 \\
\text { Powell } 1987\end{array}$ \\
\hline $\begin{array}{l}\text { Populus tremuloldes. } \\
\text { Ables lasiocerpel } \\
\text { Thallctrum fendiell C.T. }\end{array}$ & $\begin{array}{l}\text { Mountalns of } \\
\text { southeestern } \\
\text { ldaho, western } \\
\text { Wyoming, Ulah, } \\
\text { and eastern } \\
\text { Nevada } \\
(7,000 \cdot 10,100)\end{array}$ & Cool molst & $\begin{array}{l}\text { P. tremuloldes } \\
\text { seral to } \\
\text { A. lastocerpe } \\
\text { P. engeimennll }\end{array}$ & $\begin{array}{l}\text { A. leslocarpe } \\
\text { P. engalmennill }\end{array}$ & $\begin{array}{l}\text { T. lendlerl } \\
\text { P. myrsiniles } \\
\text { S. oreophlius } \\
\text { B. carinatus } \\
\text { C. rossil } \\
\text { G. viscosissimum } \\
\text { O. chliensis }\end{array}$ & Mueggler 1987 \\
\hline $\begin{array}{l}\text { Populus Iremuloldes- } \\
\text { Plnus contortal } \\
\text { Thallictrum fendierl C.T. }\end{array}$ & $\begin{array}{l}\text { Mounlalns of } \\
\text { northern Ulah } \\
(7.100-8,900)\end{array}$ & $\begin{array}{l}\text { Warm molst } \\
\text { to well. } \\
\text { dralnad }\end{array}$ & $\begin{array}{l}\text { P. Iremuloldes } \\
\text { seral to unknown } \\
\text { ultimate cllmax. } \\
\text { Probably } \\
\text { A. laslocarpe }\end{array}$ & $\begin{array}{l}\text { A. laslocarpa } \\
\text { P. contorta }\end{array}$ & $\begin{array}{l}\text { T. lendieri } \\
\text { S. Oreophilus } \\
\text { A. trachycaulum } \\
\text { E. glaucus } \\
\text { G. viscosissimum } \\
\text { O. chilens/s }\end{array}$ & Mueggler 1987 \\
\hline $\begin{array}{l}\text { Populus tremuloldes/ } \\
\text { Thallctrum fendlerl H.T. } \\
\text { (CO, SE WY) C.T.(CO, W WY.IO, } \\
\text { NE.UT) } \\
\text { [P. Iremuloldes/T. lendierl. } \\
\text { Carex geyerl H.T.(CO)] } \\
\text { T. lendlerl (Iyplc) phase } \\
\text { Delphinlum barbeyl phase (CO) } \\
\text { Ligusticum porterl phese (CO) }\end{array}$ & $\begin{array}{l}\text { Mountalns ol } \\
\text { southeastern } \\
\text { Idaho, Uleh, } \\
\text { eastern Nevade, } \\
\text { western and } \\
\text { southeastern } \\
\text { Wyoming, and } \\
\text { Colorado } \\
(8,000-10,500)\end{array}$ & Gool molst & $\begin{array}{l}\text { P. Iremuloides } \\
\text { climax (SE Wr, } \\
\text { CO). Steble or } \\
\text { serel to unknown } \\
\text { ultimete cllmax } \\
\text { (FR CO, ID, NE, } \\
\text { UT). Probably } \\
\text { A. lasiocerpe } \\
\text { P. menzlesil }\end{array}$ & $\begin{array}{l}\text { Usually pure stands. } \\
\text { May contaln } \\
\text { A. leslocarpa } \\
\text { P. menzles } \\
\text { P. Hexllts } \\
\text { P. contorta }\end{array}$ & $\begin{array}{l}\text { T. lendierl } \\
\text { S. oreophlius } \\
\text { B. cllatus } \\
\text { E. glaucus } \\
\text { C. geyerl } \\
\text { D. barbeyl } \\
\text { G. richardsonll } \\
\text { L. leucanthus } \\
\text { Llgusticum fllicinum } \\
\text { L. porterl } \\
\text { L. argenteus }\end{array}$ & $\begin{array}{l}\text { Alexander et al. } \\
\text { 1986 } \\
\text { Hass and } \\
\text { Alexandar 1986 } \\
\text { Hess and Wassar } \\
1982 \\
\text { Hollman } 1988 \\
\text { Hoftman and } \\
\text { Alexander 1980, } \\
1983 \\
\text { Komerkova el al. } \\
1988 \\
\text { Johnsion and } \\
\text { Hendzel 1985 } \\
\text { Mueggler } 1987 \\
\text { Powell } 1987\end{array}$ \\
\hline $\begin{array}{l}\text { Populus tremuloldes/ } \\
\text { Thermopsis divaricarpe C.T. }\end{array}$ & $\begin{array}{l}\text { Front Range, } \\
\text { centrel Colorado } \\
(9,600 \cdot 10,400)\end{array}$ & Cool moist & $\begin{array}{l}\text { P. Iremuloldes } \\
\text { seral to } \\
\text { A. concolor } \\
\text { P. menzlesil } \\
\text { (low elevations) } \\
\text { A. laslocarpe } \\
\text { P. engelmannll } \\
\text { (high elevatlons) }\end{array}$ & $\begin{array}{l}\text { A. concolor } \\
\text { P. menzleshl } \\
\text { A. laslocarpe } \\
\text { P. ongelmennll } \\
\text { P. contorla }\end{array}$ & $\begin{array}{l}\text { T. divericerpa } \\
\text { J. communis } \\
\text { R. woodsil } \\
\text { C. loenee } \\
\text { A. millelollum } \\
\text { F. ovalls } \\
\text { (F. virgintane) }\end{array}$ & Powell 1987 \\
\hline $\begin{array}{l}\text { Populus Iremuloldes/ } \\
\text { Veralrum renuipelalum H.T.(CO) } \\
\text { [P. Iremuloldesl } \\
\text { V. calllornicum C.T.(NE,UT)] }\end{array}$ & $\begin{array}{l}\text { Mountalns of } \\
\text { northweslern } \\
\text { Colorado, } \\
\text { northern Utah, } \\
\text { and eastern } \\
\text { Nevada } \\
(7,000-8,000)\end{array}$ & Cool molst & $\begin{array}{l}\text { P. Iremulo/des } \\
\text { cllmex (CO). } \\
\text { Stoble or seral } \\
\text { to unknown } \\
\text { ullimele cllmex } \\
\text { (NE,UT) }\end{array}$ & Usuatly pure stends & $\begin{array}{l}\text { V. calllornicum } \\
\text { V. lenulpetalum } \\
\text { B. clllatus } \\
\text { Poa elplnum } \\
\text { Carex hoodll } \\
\text { L. porterl } \\
\text { M. arizonica } \\
\text { Mertensla clllata } \\
\text { S. lameslana }\end{array}$ & $\begin{array}{l}\text { Hoflman and } \\
\text { Alexander } 1980 \\
\text { Mueggler } 1987\end{array}$ \\
\hline $\begin{array}{l}\text { Populus lremuloldes. } \\
\text { Ables lasiocarpal } \\
\text { Tall forb C.T. }\end{array}$ & $\begin{array}{l}\text { Mountalns of } \\
\text { southeaslern } \\
\text { Idaho, weslern } \\
\text { Wyoming, Utah, } \\
\text { and eastern Nevada } \\
(6,800 \cdot 10,200)\end{array}$ & $\begin{array}{l}\text { Warm well. } \\
\text { dralned }\end{array}$ & $\begin{array}{l}\text { P. tremuloides } \\
\text { serel lo } \\
\text { A. lasiocerpa } \\
\text { P. engelmannll }\end{array}$ & $\begin{array}{l}\text { A. laslocarpa } \\
\text { P. ongeimannill }\end{array}$ & $\begin{array}{l}\text { B. carinetus } \\
\text { E. glaucus } \\
\text { A. engelmannll } \\
\text { Delphinium occidentalls } \\
\text { Osmorhiza spp. } \\
\text { R. occidentalls } \\
\text { Valeriana occidentells }\end{array}$ & Mueggler 1987 \\
\hline $\begin{array}{l}\text { Populus tramuloldes/ } \\
\text { Tall forb C.T. }\end{array}$ & $\begin{array}{l}\text { Mountalns of } \\
\text { western Wyoming. } \\
\text { Uteh, and } \\
\text { eastern Neveda } \\
(7,000 \cdot 9,000)\end{array}$ & $\begin{array}{l}\text { Werm moisl } \\
\text { to well. } \\
\text { drelned }\end{array}$ & $\begin{array}{l}\text { P. tremuloides } \\
\text { stoble or serel } \\
\text { to } \\
\text { A. laslocarpa }\end{array}$ & A. lastocerpa & $\begin{array}{l}\text { Agastache uricllolla } \\
\text { A. engelmannll } \\
\text { O. occldenlalls } \\
\text { Hackella lloribunda } \\
\text { H. sphondyllium } \\
\text { (H. lanalum) } \\
\text { M. erizonica }\end{array}$ & Mueggler 1987 \\
\hline $\begin{array}{l}\text { Populus tremuloldes. } \\
\text { Pinus ponderosa C.T. }\end{array}$ & $\begin{array}{l}\text { Mountalns of } \\
\text { Utan } \\
(8,000-8,900)\end{array}$ & Warm dry & $\begin{array}{l}\text { P. tremuloldes } \\
\text { serel to } \\
\text { P. ponderosa }\end{array}$ & P. ponderosa & $\begin{array}{l}\text { 8. repens } \\
\text { J. communis } \\
\text { Q. gambell } \\
\text { S. oreophilus } \\
\text { P. fendlerlana } \\
\text { A. millefollum } \\
\text { T. ollicinale }\end{array}$ & Mueggler 1987 \\
\hline $\begin{array}{l}\text { Popuius tramuloldes. } \\
\text { Pinus Hoxills C.T. }\end{array}$ & $\begin{array}{l}\text { Mountalns of } \\
\text { western Wyoming. } \\
\text { Utah, and eestern } \\
\text { Neveda }(\geq 9,000)\end{array}$ & Cool dry & $\begin{array}{l}\text { P. Iremuloldes } \\
\text { usually steble. } \\
\text { Mey be } \\
\text { succeeded by } \\
\text { P. flexills }\end{array}$ & P. $\|$ exllls & $\begin{array}{l}\text { 8. repens } \\
\text { S. oreophilus } \\
\text { A. Irachycaulum } \\
\text { C. rossll } \\
\text { A. millatolium } \\
\text { S. jameslana }\end{array}$ & Mueggler 1987 \\
\hline
\end{tabular}





\begin{tabular}{|c|c|c|c|c|c|c|}
\hline $\begin{array}{l}\text { Hebltet type or } \\
\text { community type }\end{array}$ & $\begin{array}{l}\text { Locatlon and } \\
\text { elevetlon (feet) }\end{array}$ & Slte & $\begin{array}{l}\text { Succassional } \\
\text { etetus }\end{array}$ & $\begin{array}{c}\text { Tree } \\
\text { assoclates }\end{array}$ & $\begin{array}{l}\text { Principal undergrowth } \\
\text { specles }\end{array}$ & Authority \\
\hline $\begin{array}{l}\text { Populus tremuloldes. } \\
\text { Picea pungens C.T. }\end{array}$ & $\begin{array}{l}\text { Mountalns of } \\
\text { Uteh } \\
(7,400-9,100)\end{array}$ & Cool dry & $\begin{array}{l}\text { P. Iremuloldes } \\
\text { seral to } \\
\text { P. pungens }\end{array}$ & P. pungens & $\begin{array}{l}\text { J. communis } \\
\text { S. oreophllus } \\
\text { B. enomalus } \\
\text { P. pretensis } \\
\text { A. millefollum }\end{array}$ & Mueggler 1987 \\
\hline
\end{tabular}

Pinus contorta series end other $P$. contorto-domineted vegetetion

Pinus contortal A rctostaphylos uva.urs/ H.T.

Pinus contortal

Berberis repens C.T.

Pinus contortal Juniperus communis H.T.(CO SE WY:CT ID NW WY, UT

Mountelns of
northern Utah
and north-centra
Wyoming; Front
Range, Colorado
$(7,800-9,500)$
Mountalns of
northern Uteh
$(7,700-10,000)$

Mountalns of southeastern Idaho northwestern and southeastern Wyoming, norther
Utah, and central and north-central Colorado $(8,000 \cdot 10,500)$

\section{Pinus contortal}

Uinneea porealis C.T.

Mountains of Montena east of Continental northwest $(5,6007,200)$

Pinus contortal Purshie tridentale H.T.

Mountalns of $(\leq 6,600)$

\begin{tabular}{|c|c|c|}
\hline $\begin{array}{l}\text { Warm dry } \\
\text { to well- } \\
\text { drelned }\end{array}$ & $\begin{array}{l}\text { P. contorla } \\
\text { climex or stable }\end{array}$ & $\begin{array}{l}\text { Usuelly pure stends. } \\
\text { May conteln } \\
\text { P. tremuioides }\end{array}$ \\
\hline $\begin{array}{l}\text { Cool dry } \\
\text { to well. } \\
\text { dralned }\end{array}$ & $\begin{array}{l}\text { P. contorla } \\
\text { seral to unknown } \\
\text { ultimate cllmax. } \\
\text { Probebly } \\
\text { A. leslocerpa } \\
\text { A. concolor }\end{array}$ & $\begin{array}{l}\text { Usually } \\
\text { P. Iremuloldes. } \\
\text { Mey elso contaln } \\
\text { A. lasiocerpe } \\
\text { A. concolor }\end{array}$ \\
\hline Cool dry & $\begin{array}{l}\text { P. contorta } \\
\text { climax (CO,SE WY) } \\
\text { or seral to } \\
\text { unknown ultimate } \\
\text { cflmax (ID,NWWY). } \\
\text { Probably } \\
\text { A. fasiocarpa } \\
\text { P. menzles:l }\end{array}$ & $\begin{array}{l}\text { Usually pure stands. } \\
\text { Mey contaln } \\
\text { A. laslocarpa } \\
\text { P. menzlesil } \\
\text { P. engeimannll } \\
\text { P. ponderosa } \\
\text { P. afblcaulls } \\
\text { P. tremuloldes }\end{array}$ \\
\hline $\begin{array}{l}\text { Cool molst } \\
\text { to well. } \\
\text { dralned }\end{array}$ & $\begin{array}{l}\text { P. contorte } \\
\text { seral to unknown } \\
\text { ultimate climax. } \\
\text { Probably } \\
\text { A. laslocarpa } \\
\text { P. menzlesill }\end{array}$ & $\begin{array}{l}\text { A. lasiocarpa } \\
\text { P. menzies"l } \\
\text { P. engeimannll }\end{array}$ \\
\hline $\begin{array}{l}\text { Warm dry } \\
\text { to well. } \\
\text { dralned }\end{array}$ & $\begin{array}{l}\text { P. contorte } \\
\text { climax or steble }\end{array}$ & $\begin{array}{l}\text { Usually pure stands. } \\
\text { Mey contefn } \\
\text { A. leslocarpa } \\
\text { P. menzies" } \\
\text { P. engelmannil } \\
\text { P. tremuloldes }\end{array}$ \\
\hline $\begin{array}{l}\text { Warm dry } \\
\text { to well. } \\
\text { dralned }\end{array}$ & $\begin{array}{l}\text { P. contorte } \\
\text { climax (CO,SE WY) } \\
\text { or seral to } \\
\text { unknown ultimate } \\
\text { climax (IO,NWWY). } \\
\text { Probably } \\
\text { A. lastocarpa } \\
\text { P. menzios"l }\end{array}$ & $\begin{array}{l}\text { Usually pure stands. } \\
\text { May contain } \\
\text { A. lasiocarpe } \\
\text { P. menzlesil } \\
\text { P. engeimennll } \\
\text { P. tremuloides }\end{array}$ \\
\hline Warm dry & $\begin{array}{l}\text { P. contorta } \\
\text { seral to unknown } \\
\text { uttlmate ctimax. } \\
\text { Probably } \\
\text { A. lasiocarpa }\end{array}$ & $\begin{array}{l}\text { A. laslocarpa } \\
\text { P. engeimanntl } \\
\text { P. menziestl }\end{array}$ \\
\hline $\begin{array}{l}\text { Cool molst } \\
\text { to well. } \\
\text { drained }\end{array}$ & $\begin{array}{l}\text { P. contorta } \\
\text { seral to unknown } \\
\text { ultimate cllmax. } \\
\text { Probably } \\
\text { A. taslocarpa } \\
\text { P. menzlesll }\end{array}$ & $\begin{array}{l}\text { A. lesiocerpe } \\
\text { P. menziesil } \\
\text { P. engelmennil }\end{array}$ \\
\hline $\begin{array}{l}\text { Cool well. } \\
\text { drained }\end{array}$ & $\begin{array}{l}\text { P. contorta } \\
\text { seral to unknown } \\
\text { ultimate cllmax. } \\
\text { Probably } \\
\text { A. laslocarpe } \\
\text { P. menzlesil }\end{array}$ & $\begin{array}{l}\text { A. lasiocarpa } \\
\text { P. menziesil } \\
\text { P. engeimannil }\end{array}$ \\
\hline Cool dry & $\begin{array}{l}\text { P. contorta } \\
\text { climax }\end{array}$ & P. tremuloides \\
\hline Cool dry & $\begin{array}{l}\text { P. contorte } \\
\text { cllmex (CO,SE WY) } \\
\text { or seral to } \\
\text { unknown ultimate } \\
\text { climax (ID,NW WY. } \\
\text { UT). Probably } \\
\text { A. lasiocarpa } \\
\text { A. grandis } \\
\text { P. menziesil }\end{array}$ & $\begin{array}{l}\text { Usually pure slands. } \\
\text { May contaln } \\
\text { A. lasiocerpa } \\
\text { P. menzlesil } \\
\text { A. grandis } \\
\text { P. engelmennil } \\
\text { P. Ilexilis } \\
\text { P. albicaulis } \\
\text { L. occldentalis } \\
\text { T. helerophylie }\end{array}$ \\
\hline
\end{tabular}

A. uva.urst

J. communis

S. betulliolle

. hystrix
c. rossil

B. repens

A. peluta

P. myrsinites

C. geyeri

A. cordifolla

J. communis

A. uva.ursi

B. repens
S. canadensis

S. canadensis
A. cordilolla

Lupinus spp.

Luplnus spp.

L. borealls

V. globulare

V. scoparium

A. cordllollo

A. cordlolla

P. tridentata

A. uva.ursl

A. splcalum
F. Idahoensis

C. rossil

Eplioblum angusllollum

Pinus contortal

Shepherdie canadensis $H_{\text {T }}$.

Mounteins of

southeastern fdaho

7,000-8,000),

northwestern and

Wyoming, and north

central Cotorado

$(8,000-9,800)$

Pinus contorta/
Splraea betuiliolis C.T.

Mountains

southeastern ldaho

and northw
Wyoming

(7.000-8.000)

Pinus contortal

Mountains of

south.centrat

$(5,000-7,500)$,

and northern

Utah

$300 \cdot 10,000)$

Pinus contortal

Vaccinium giobulare C.T

Mountains of

southeastern Idaho,

northwestern

Wyoming, and

$(7,500 \cdot 9,000)$

$\begin{array}{ll}\text { Pinus contortel } & \text { Front Range } \\ \text { Vaccinlum myrtilius H.T. } & \text { Colorado }\end{array}$

Front Range,

$(8,500 \cdot 10,000)$

Plinus contort

Mountains of

Vaccinlum scoperium H.T. $\quad$ Montana, Idaho,

Wyoming $(7,000$

8,500), northern

Utah, southeastern

Wyoming, an

western and

central Colorado
Hoflman and

Alexander 1976

Meuk and 1984

Henderson 1984
Radloff 1983

Mauk and

Henderson 1984

Alexander et al.

1986

Alexandar 1986

Komerkova el al.

1988

Mauk and

Henderson
Aadloff 1983

Steele of al. 1983

Pflster et al. 1977

Steele et al. 1983

Pftster et al. 1977

P. contorta Usually puro

seral lo unknown May contaln

ultimate cllmax. A. lesiocerpa

$\begin{array}{ll}\text { Probably } & \text { P. menziesh } \\ \text { A. laslocarpe } & \text { P. engolmannil }\end{array}$

P. menzlesill L. occidentalls

Utah, and

northweste
Wyoming

$(6,000 \cdot 8,000)$

Pinus contortal

Mountalns of

central Ideho

$(5,000-9,000)$

Mountelns of

central Idaho,

northwestern

Wyoming $(8,000$

9,000), soulh.

eastern Wyoming,

Colorado

$(7,500 \cdot 10,000)$
Warm dry

to well.
dralned

contorto

P. albicaulis

P. elbicaulls

minor climex

Cool dry

P. contorta

climex (CO) or

serel to unknow

(ID,WY).

A. laslocarpe

P. menziesil

ay contal

A. lasiocerpe

$P$. engelmann

P. albicaulls

P. Iremuloldas

P. contorta P. elbicaulls

F. Idahoensis

C. rossil

Artemisla spp.

C. geyeri

J. communis

P. myrsiniles

R. woodsil

S. oreophllus

Fragarla $\mathrm{spp}$.

L. argenteus
S. canedensis

A. uve-ursi

$\checkmark$. commun

L. borealls

P. myrsinites
R. woodsil

V. scoparlum
A. cordifolla

s. betulifolla

S. myrsinites

S. oreophilus

C. geyeri

V. caespitosum

J. communis

L. boreells

V. scoparium

Festuca ovina

V. globulare

v. scoparium

C. rubescens

V. myrtillus

L. borealls

C. geyerl

V. scoparlum

A. uva.ursi

8. repens

. borealls

c. rubescens

C. geyer

rossil

cordilolla

ergenteus

canadensis

J. communis

. nervose

A. cordifolla

C. rubescens

A. uva-urs

S. albus

C. geyerl

Alexander et al.

1986

Hess and

Hess and Wasser

1982

Hoffman an

Alexander 1980
Steole of at. 1983

Steele et al. 1983

Cooper et al. 1987

Mauk and

Ptister el al. 1977

Steele el al. 1981

Steele et al. 1983
Henderson 198
Padloll 1983

Alexander et al.

1986

Hess and

Holfman and

Alexander 1980

1988

Mauk and

Henderson 1984

Henderson 1984
Pfister et al. 1977

Steele et al.

1981, 1983

Mauk and

Henderson 1984

Pflster el al. 1977

Steele et al. 1983

Steele et al. 1981

Ste

Alexander et el.

1986

Hess and

Alexender 1986

Hess and Wesser

1982

Komerkova et al.

1988

Steele et al.
1981,1983
Cooper el el. 1987

Alexander 1986 



\begin{tabular}{|c|c|c|c|c|c|c|}
\hline $\begin{array}{l}\text { Hebltet lype or } \\
\text { community lype }\end{array}$ & $\begin{array}{l}\text { Locetion and } \\
\text { elevetion (toet) }\end{array}$ & Sito & $\begin{array}{l}\text { Successlonal } \\
\text { stetus }\end{array}$ & $\begin{array}{c}\text { Tree } \\
\text { essocletes }\end{array}$ & $\begin{array}{l}\text { Princlpal undergrowth } \\
\text { spectes }\end{array}$ & Authorlty \\
\hline $\begin{array}{l}\text { Pinus contorlal } \\
\text { Carex rossl/ H.T.(UT,SE WY); } \\
\text { C.T.(NWW WY) }\end{array}$ & $\begin{array}{l}\text { Mountelns of } \\
\text { norlhern Ulah, } \\
\text { end northwestern } \\
\text { and soulheeslern } \\
\text { Wyoming } \\
(8,500 \cdot 10,000)\end{array}$ & Cool dry & $\begin{array}{l}\text { P. contorta } \\
\text { cllmex (SE WY,UT) } \\
\text { or seral to } \\
\text { unknown ultimate } \\
\text { cllimax (NW WY). } \\
\text { Probably } \\
\text { A. laslocarpo } \\
\text { P. engelmannll }\end{array}$ & $\begin{array}{l}\text { May be pure slends. } \\
\text { Usuelly conlaln } \\
\text { A. laslocrpa } \\
\text { P. engelmannll } \\
\text { P. albicaulls } \\
\text { P. lremuloides }\end{array}$ & $\begin{array}{l}\text { C. rossil } \\
\text { P. nervosa } \\
\text { L. argenteus } \\
\text { Pyrola spp. } \\
\text { Sadum lancaolatum } \\
\text { Salldago multiradlata }\end{array}$ & $\begin{array}{l}\text { Alexendar et el. } \\
1986 \\
\text { Mauk and } \\
\text { Henderson } 1984 \\
\text { Sleele el al. } 1983\end{array}$ \\
\hline $\begin{array}{l}\text { Pinus contortal } \\
\text { Ainica cordilfolla C.T. }\end{array}$ & $\begin{array}{l}\text { Mounlatns of } \\
\text { soulheestern Idaho } \\
\text { and northweslern } \\
\text { Wyoming } \\
(6,000 \cdot 9,000)\end{array}$ & Cool dry & $\begin{array}{l}\text { P. contorta } \\
\text { seral lo unknown } \\
\text { ullimate cllmax. } \\
\text { Probably } \\
\text { A. lasiocarpa } \\
\text { P. engeimannll } \\
\text { P. menzlesil }\end{array}$ & $\begin{array}{l}\text { Usuelly pure slands. } \\
\text { Mey conteln } \\
\text { A. laslocarpa } \\
\text { P. engelmannill } \\
\text { P. menzlesil } \\
\text { P. alblcaulls } \\
\text { P. flexills }\end{array}$ & $\begin{array}{l}\text { A. cordifolls } \\
\text { A. racemosa } \\
\text { A. miser } \\
\text { P. secunda }\end{array}$ & Steele el al. 1983 \\
\hline $\begin{array}{l}\text { Pinus contortal } \\
\text { Xerophyllum tenex C.T. }\end{array}$ & $\begin{array}{l}\text { Mountelns of } \\
\text { northern Idaho } \\
(6,000-8,000)\end{array}$ & Cool dry & $\begin{array}{l}\text { P. contorta } \\
\text { serel to } \\
\text { A. lastocarpa }\end{array}$ & $\begin{array}{l}\text { A. laslocarpa } \\
\text { P. engelmannll } \\
\text { P. menzlesil }\end{array}$ & $\begin{array}{l}\text { X. tenax } \\
\text { V. globutare } \\
\text { V. scoparlum }\end{array}$ & Cooper et al. 1987 \\
\hline \multicolumn{7}{|c|}{ Pinus aristata series } \\
\hline $\begin{array}{l}\text { Pinus arisietal } \\
\text { Juniperus communis H.T. }\end{array}$ & $\begin{array}{l}\text { Mountalns of } \\
\text { soulh.centrel } \\
\text { Coloredo } \\
(9,500 \cdot 10,000)\end{array}$ & Cool dry & $\begin{array}{l}\text { P. arlstate } \\
\text { climex or } \\
\text { co.cllmax with } \\
\text { P. menzles } I\end{array}$ & $\begin{array}{l}\text { P. manzias } \\
\text { P. flexills } \\
\text { P. Iramuloldes }\end{array}$ & $\begin{array}{l}\text { J. communis } \\
\text { A. uva-urst } \\
\text { Artem/sla spp. } \\
\text { M. montana } \\
\text { P. tendleriana }\end{array}$ & $\begin{array}{l}\text { Komarkova el al. } \\
1988\end{array}$ \\
\hline $\begin{array}{l}\text { Pinus aristafal } \\
\text { Rlbes monllgenum H.T. } \\
\text { (Scree torest) }\end{array}$ & $\begin{array}{l}\text { Mountelns of } \\
\text { northern New } \\
\text { Mexico and } \\
\text { soulhern Colorado } \\
(11,000.11,500)\end{array}$ & Cool dry & $\begin{array}{l}\text { P. aristate } \\
\text { cllmax }\end{array}$ & $\begin{array}{l}\text { Usually pure slands. } \\
\text { May contaln } \\
\text { P. engelmannll }\end{array}$ & $\begin{array}{l}\text { R. montigenum } \\
\text { S. bronchialis }\end{array}$ & $\begin{array}{l}\text { Devellce et al. } \\
1986\end{array}$ \\
\hline $\begin{array}{l}\text { Pinus artstatal } \\
\text { Festuca arlzonica H.T. }\end{array}$ & $\begin{array}{l}\text { Mountelns of } \\
\text { northern New } \\
\text { Mexico, and } \\
\text { soulhern ond } \\
\text { weslern Colorado } \\
(8.600-10,000)\end{array}$ & Werm dry & $\begin{array}{l}\text { P. aristale } \\
\text { climex or } \\
\text { co.cllmax with } \\
\text { P. menzlesil }\end{array}$ & $\begin{array}{l}\text { P. menziesh } \\
\text { P. llaxills } \\
\text { P. tremuloldes }\end{array}$ & $\begin{array}{l}\text { F. arlzonica } \\
\text { R. cereum } \\
\text { K. cristata } \\
\text { (K. macrantha) } \\
\text { M. montana } \\
\text { A. friglda }\end{array}$ & $\begin{array}{l}\text { Devellce et al. } \\
1986 \\
\text { Komarkova et al. } \\
1988\end{array}$ \\
\hline $\begin{array}{l}\text { PInus aristatal } \\
\text { Fesfuca thurberi H.T. }\end{array}$ & $\begin{array}{l}\text { Mountains of } \\
\text { northern New } \\
\text { Mexlco and } \\
\text { southern and } \\
\text { western Colorado } \\
(10,000 \cdot 11,800)\end{array}$ & Cool dry & $\begin{array}{l}\text { P. aristala } \\
\text { cllmax or } \\
\text { co.cllmax with } \\
P . \text { engelmannll }\end{array}$ & $\begin{array}{l}\text { P. engelmannll } \\
\text { P. tremuloides }\end{array}$ & $\begin{array}{l}\text { F. thurbarl } \\
\text { A. monllganum } \\
\text { A. cordlfolla } \\
\text { Polemonlum puicherrimum } \\
\text { (P. dellcalum) } \\
\text { S. bronchialls }\end{array}$ & $\begin{array}{l}\text { DeVellce el al. } \\
1986 \\
\text { Komarkova el al. } \\
1988\end{array}$ \\
\hline $\begin{array}{l}\text { PInus arlstatal } \\
\text { Trilfollum dasyphyllum H.T. }\end{array}$ & $\begin{array}{l}\text { Mounlains of } \\
\text { north-cenlral } \\
\text { Colorado } \\
(11,400-11,600)\end{array}$ & Cool dry & $\begin{array}{l}\text { P. aristala } \\
\text { climax. } \\
\text { P. engelmannll } \\
\text { minor climax }\end{array}$ & P. engalmannil & $\begin{array}{l}\text { T. dasyphyllum } \\
\text { C. foenea } \\
\text { A. lanulosa } \\
\text { Penslemon whippleanus } \\
\text { P. pulcherrimum } \\
\text { (P. delicalum) }\end{array}$ & $\begin{array}{l}\text { Hess and } \\
\text { Alexander } 1986\end{array}$ \\
\hline \multicolumn{7}{|c|}{ Picea glauca series } \\
\hline $\begin{array}{l}\text { Plcea glaucal } \\
\text { Linnaea borealls H.T. }\end{array}$ & $\begin{array}{l}\text { Black Hitts } \\
\text { and Bear Lodge } \\
\text { Mountalns. South } \\
\text { Dakota and } \\
\text { easlern Wyoming } \\
(5,800-6,500)\end{array}$ & $\begin{array}{l}\text { Coot well- } \\
\text { dralned }\end{array}$ & $\begin{array}{l}\text { P. glauca } \\
\text { climax }\end{array}$ & $\begin{array}{l}\text { P. ponderosa } \\
\text { P. tromulaldes } \\
\text { B. popyritora }\end{array}$ & $\begin{array}{l}\text { L. Doreal/s } \\
\text { J. communis } \\
\text { Rosa acicularis } \\
\text { O. asporillila } \\
\text { F. ovalls } \\
\text { (F. virgintana) }\end{array}$ & $\begin{array}{l}\text { Hollman and } \\
\text { Alexander } 1987\end{array}$ \\
\hline $\begin{array}{l}\text { Picas glaucal } \\
\text { Vacctnlum scoparium H.T. }\end{array}$ & $\begin{array}{l}\text { Black HIIIs } \\
\text { and Bear Lodge } \\
\text { Mountalns, South } \\
\text { Dakote and } \\
\text { eastern Wyoming } \\
(5,700-6,700)\end{array}$ & $\begin{array}{l}\text { Cool well. } \\
\text { drained }\end{array}$ & $\begin{array}{l}\text { P. glauca } \\
\text { climex }\end{array}$ & $\begin{array}{l}\text { P. ponderosa } \\
\text { P. Pramutoldes } \\
\text { B. papyritera }\end{array}$ & $\begin{array}{l}\text { V. scoparlum } \\
\text { 8. repens } \\
\text { J. communis } \\
\text { S. belullfolla } \\
\text { F. ovalis } \\
\text { (F. virginiana) } \\
\text { G. boreale }\end{array}$ & $\begin{array}{l}\text { Holtman and } \\
\text { Alexander } 1987\end{array}$ \\
\hline $\begin{array}{l}\text { Plcea engelmannill } \\
\text { Acer glabrum H.T. }\end{array}$ & $\begin{array}{l}\text { Mountalns ol } \\
\text { soulh.cantral } \\
\text { Arizona and } \\
\text { soulhern } \\
\text { New Mexico } \\
(8,500-9,500)\end{array}$ & $\begin{array}{r}\text { Pic } \\
\text { Cool molst }\end{array}$ & $\begin{array}{l}\text { engelmannii se } \\
\text { P. engolmannll } \\
\text { cllmax. } \\
\text { A. laslocarpa } \\
P \text {. angeimannll } \\
\text { minor ctimaxes }\end{array}$ & $\begin{array}{l}\text { Aries } \\
\text { A. laslocarpa } \\
\text { P. engeimannll } \\
\text { P. menzlesll } \\
\text { A. concolor } \\
\text { P. stroblformis } \\
\text { P. tremuloldes }\end{array}$ & $\begin{array}{l}\text { A. glabrum } \\
\text { B. clllatus } \\
\text { L. portarl } \\
\text { S. stallata } \\
\text { V. canadensis }\end{array}$ & $\begin{array}{l}\text { Alexander et al. } \\
1984 e \\
\text { DeVellce and } \\
\text { Ludwig } 1983 \\
\text { Molr and } \\
\text { Ludwig } 1979\end{array}$ \\
\hline $\begin{array}{l}\text { Plcee engelmannill } \\
\text { Junlperus communis H.T. }\end{array}$ & $\begin{array}{l}\text { Mounlalns ol } \\
\text { northweslern } \\
\text { Wyoming } \\
(7,400 \cdot 10,300)\end{array}$ & Cool dry & $\begin{array}{l}\text { P. angalmannll } \\
\text { cllmax }\end{array}$ & $\begin{array}{l}\text { P. manziesill } \\
\text { P. contorta } \\
\text { P. flexills } \\
\text { P. albicaulls }\end{array}$ & $\begin{array}{l}\text { J. communls } \\
\text { A. cordifolla } \\
\text { Frasara speclosa } \\
\text { S. mulliradiata }\end{array}$ & Slaale et al. 1983 \\
\hline $\begin{array}{l}\text { Plces engelmannill' } \\
\text { Linnaes borealls H.T. }\end{array}$ & $\begin{array}{l}\text { Mountalns of } \\
\text { Montena easl ol } \\
\text { Continental } \\
\text { Oivide }(4,200 \text {. } \\
7.800) \text {, and } \\
\text { northwestern } \\
\text { Wyoming } \\
(8,200 \cdot 8,200)\end{array}$ & $\begin{array}{l}\text { Cool well- } \\
\text { dralned }\end{array}$ & $\begin{array}{l}\text { P. ongolmannll } \\
\text { cllmax }\end{array}$ & $\begin{array}{l}\text { P. menzlasll } \\
\text { P. contorts }\end{array}$ & $\begin{array}{l}\text { L. boraalls } \\
\text { J. communis } \\
\text { S. albus } \\
\text { V. globulara } \\
\text { C. rubescens } \\
\text { A. cordllolla } \\
\text { P. secunda }\end{array}$ & $\begin{array}{l}\text { Pflsler et el. } 1977 \\
\text { Steele el al. } 1983\end{array}$ \\
\hline $\begin{array}{l}\text { Picea angelmannil' } \\
\text { Physocarpus malvacaus H.T. }\end{array}$ & $\begin{array}{l}\text { Mountalns of } \\
\text { south.centrel } \\
\text { Monlana, } \\
\text { soulheastern idaho. } \\
\text { and northwestern } \\
\text { Wyoming } \\
(5,900 \cdot 7,200)\end{array}$ & Cool molsl & $\begin{array}{l}\text { P. engelmannll } \\
\text { climax. } \\
\text { A. lasiocarpa } \\
\text { minor climex }\end{array}$ & $\begin{array}{l}\text { A. taslocarpa } \\
\text { P. manzlesil } \\
\text { P. contorta }\end{array}$ & $\begin{array}{l}\text { P. malvaceus } \\
\text { S. belullfolla } \\
\text { S. albus } \\
\text { G. Iriflorum } \\
\text { Thallctrum spp. }\end{array}$ & $\begin{array}{l}\text { Pilster et al. } 1977 \\
\text { Steele el al. } 1983\end{array}$ \\
\hline $\begin{array}{l}\text { Pices engeimannil' } \\
\text { Albes monilgenum H.T. }\end{array}$ & $\begin{array}{l}\text { Mountalns of } \\
\text { northwestern } \\
\text { Wyoming }(8,400 \\
9,700) \text { and } \\
\text { southarn Utah } \\
(10,000-11,400)\end{array}$ & Cool dry & $\begin{array}{l}\text { P. engelmannll } \\
\text { climax }\end{array}$ & $\begin{array}{l}\text { P. contorta } \\
\text { P. albicaulis } \\
\text { P. } l \text { exills } \\
\text { P. tromuloides }\end{array}$ & $\begin{array}{l}\text { R. montlgenum } \\
\text { F. ovina } \\
\text { A. caarulea } \\
\text { A. latiolia } \\
\text { A. miser } \\
\text { Sibbaldla procumbens }\end{array}$ & $\begin{array}{l}\text { Plisler } 1972 \\
\text { Steele el al. } 1983 \\
\text { Youngblood and } \\
\text { Meuk } 1985\end{array}$ \\
\hline $\begin{array}{l}\text { Picea engelmannill } \\
\text { Sallx pseudolapponum H.T. } \\
\text { [P. engelmannill } \\
\text { Ables laslocarpal } \\
\text { S. Pseudolapponum H.T.J }\end{array}$ & $\begin{array}{l}\text { Mountains of } \\
\text { northern end } \\
\text { central Colorado } \\
(11,200 \cdot 11,800)\end{array}$ & Cool molst & $\begin{array}{l}\text { P. angelmannill } \\
\text { climax }\end{array}$ & $\begin{array}{l}\text { A. laslocarpa } \\
\text { P. contorta } \\
\text { P. flexills }\end{array}$ & $\begin{array}{l}\text { S. pseudolapponum } \\
\text { V. scoparlum } \\
\text { G. rosill } \\
\text { P. pulcherrimum } \\
\text { (P. dellcatum) }\end{array}$ & $\begin{array}{l}\text { Hess end } \\
\text { Alexander } 1986 \\
\text { Hess and Wesser } \\
1982\end{array}$ \\
\hline $\begin{array}{l}\text { Plcea engelmannill } \\
\quad \text { Vaccinlum caespliosum H.T. }\end{array}$ & $\begin{array}{l}\text { Mountains of } \\
\text { norlhwestern } \\
\text { Montana }(3,100- \\
5,300) \text { and } \\
\text { northarn Utah } \\
(9,300-11,000)\end{array}$ & $\begin{array}{l}\text { Cool molsl } \\
\text { lo well. } \\
\text { dralned }\end{array}$ & $\begin{array}{l}\text { P. engelmannll } \\
\text { climax }\end{array}$ & $\begin{array}{l}\text { P. menzlosil } \\
\text { P. contorta } \\
P_{1} \text { ponderosa } \\
\text { L. occldenlalls } \\
P_{1} \text { tremuloldes }\end{array}$ & $\begin{array}{l}\text { V. caespllosum } \\
\text { L. borealls } \\
\text { A. monligenum } \\
\text { V. scoparium } \\
\text { C. rubescens }\end{array}$ & $\begin{array}{l}\text { Meuk end } \\
\text { Handerson } 1984 \\
\text { Pflster et al. } 1977\end{array}$ \\
\hline 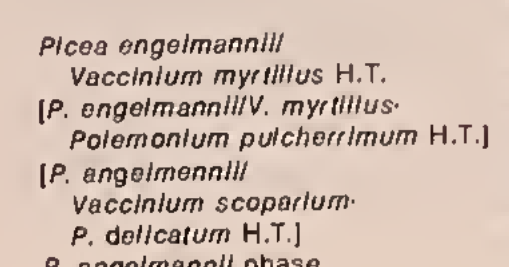 & $\begin{array}{l}\text { Mounlains of } \\
\text { northern and } \\
\text { southwestern } \\
\text { New Mexlco, and } \\
\text { southern and } \\
\text { central Colorado } \\
(9,400 \cdot 11,900)\end{array}$ & $\begin{array}{l}\text { Cool dry } \\
\text { lo well. } \\
\text { dralned }\end{array}$ & $\begin{array}{l}\text { P. engelmannll } \\
\text { climax. } \\
\text { A. lasiocarpa } \\
\text { co.cllmax, minor } \\
\text { climax or absanl }\end{array}$ & $\begin{array}{l}\text { A. laslocarpa } \\
\text { P. menzlesll } \\
\text { P. stroblformis } \\
\text { P. pungens } \\
\text { P. aristola } \\
\text { P. tromuloldes }\end{array}$ & $\begin{array}{l}\text { V. myrillus } \\
\text { J. americana } \\
\text { J. communis } \\
\text { Rosa spp. } \\
\text { V. scoparlum } \\
\text { P. pulcherrimum } \\
\text { (P. dellicalum) } \\
\text { Seneclo amplectens }\end{array}$ & $\begin{array}{l}\text { Devellce at al. } \\
1986 \\
\text { Fllzhugh el al. } \\
1987 \\
\text { Molr end } \\
\text { Ludwig } 1979 \\
\text { Radlolf } 1983\end{array}$ \\
\hline
\end{tabular}





\section{Habital lype or}

\section{cond}

Plcaa enge/mannill

Vaccinlum scoperium H.T.

\section{Picea engelmannill
Cerex loanea H.T.}

Picea engelmannil'

Arnice cordholla H.T.

Plcea engelmannill

Calthe leptosapaie H.T.

Mountalns of
Utah $(10,000$.
$11,000)$, north.
weslern Wyoming.
and southeastern

and southeastern

Picee engelmannil

Clinsonia unitiora H.T.

C. unillora (typlc) phase
Vaccinlum coespitosum phase

Picea engelmennil'

Equiselum ervense H.T.

to

\section{Picea engelmannili}

Erigeron eximius H.T.

[P. pungens.P. enge/mennIII
E. superbus M.T.]

P/cea engelmannIII

Gallum tillorum H.T.

\section{Picea engelmannlli}

Geum ross/l H.T.

Picea engelmannill Heracleum sphondylium H.T.
(Riparian forest)

\section{Mountains of}

northwestern

Montana
$(3,000 \cdot 4,000)$

Mounlalns ol

Montana, central

ldaho $(2,900$

$6,800)$, north.
westarn Wyoming

and northern Ula

Mountains ol

eastern Arizona

and soulhweslern
New Mexico

New Mexico
$(8,500-9,500)$

Mounlains of

Soulh.central

Montana, centra

Idaho, and norin.
weslern Wyoming

$(6.000 \cdot 8,500)$

Mountains of
north-centrat

north-cen

Arizona

Mountains ol

norlhern Naw

southern Colorado

$(8,800 \cdot 9,200)$

\section{Picea engalmannili}

Hypnum revolutum H.T.

Mountains of

centrat and

soulheastern
tdaho 8,100 .

$8,300)$, and norih.

westarn Wyoming

$7.700 \cdot 10,500$ )

Picea angelmannili

Sexilraga bronchialls H.T.

Mounlains of

northern New

Mexico and
southern Colorado

southern Colorado
$(11,200 \cdot 11,800)$

Mountains of

eastarn Arizona
and southwastarn

Plcea angelmannili
Senecio cardamine H.T

(P. pungens.P angaimannili)

S. cardamina H.T.]

Ables fasiocarpa phase

Pices engalmannllI

Saneclo streptanthllollus H.T.

P. angelmannill phase

Pseudolsuga manzles/l phase

Picea engeimannili

Smllacina stallate H.T.

Picea engelmannil

Trilollum dasyphylium H.T.

New Mexico

$(8,500-9,400)$

Mountains of

ceniral and

southwestarn

Monlana

Mountalns ol

Montana aast of

Continenlal

(4.400.7.400)

Mounlains of north.

ceniral Colorado

$(10,800-11,300)$

Picea engelmannil

Moss spp. H.T.
Mountains of

Southwastern New

Arizona; Front

Range, Colorado

$(9,500 \cdot 11,000)$

Site $\begin{gathered}\text { Successlonat } \\ \text { status }\end{gathered}$

Trae

Principal undergrowth

spaclas

\begin{tabular}{|c|c|c|c|}
\hline $\begin{array}{l}\text { P. angelmannil } \\
\text { cllmax } \\
\text { A. laslocarpa } \\
\text { may ba minor } \\
\text { climax }\end{array}$ & $\begin{array}{l}\text { A. lasiocarpa } \\
\text { P. manzlasll } \\
\text { P. contorta } \\
\text { P. \|laxills } \\
\text { P. elbiceulls }\end{array}$ & $\begin{array}{l}\text { V. scoparium } \\
\text { C. rossli } \\
\text { A. cordilolla } \\
\text { Antennarla spp. } \\
\text { F. ovalls } \\
\text { (F. virginlana) } \\
\text { Lupinus spp. }\end{array}$ & $\begin{array}{l}\text { Hollman and } \\
\text { Alexander } 1976 \\
\text { Mauk and } \\
\quad \text { Handerson } 1984 \\
\text { Steele at al. } 1983\end{array}$ \\
\hline
\end{tabular}

Coot dry
to wall.
dralned

P. angelmennll

co.cllmax with P. aristate

A. leslocarpa. P. lramuloldes

P. manzlasit

P. inor climax

Coot moist

cllmax.

A. lasiocarpa

A. laslocarpa
P. pungens

P. angoimannil

Cool to

P. angalmann

cllmax.

A. laslocarpa
minor climax

dralned

Coot dry

$P$. angalmannil

A. laslocarpa

P. menzles/l
P. tremuloides

cllmax

\section{P. menzles /}

P. contorta
P. llexills

P. Ilexills
P. albicaulls

P. tramuloides

Cool molst

$\begin{array}{ll}\text { P. engelmannil } & \text { A. leslocarpe } \\ \text { cllmax. } & \text { P. contorla } \\ \text { A. lasiocarpa } & \text { P. albicaulis } \\ \text { minor cllmax } & \end{array}$

Warm molst

P. engeimannil

drained

cllmax

P. menzies /

P. ponderose

L. occldentalis

Warm to

cool wet

P. engelmannil

A. leslocarpe

cllmax.

$P$. pungens

A. teslocarpe
mInor climax

P. contorte

Cool w

P. engelmann

P. menzles/I

E. trilicoldes

A. glabrum

H. dumosis

H. amarican
Ribes spp.

Alexander et al.

Molr and

Ludwlg 1979

C. disparma

Aclas rubra

G. trillorum

Saxiltraga arguta

Saxilraga arguta
S. Irlangularls

C. loanae

B. clliatus
P. pratansis

A. cordllolla

F. Idahoensis

H. kingll

C. ross"

A. misar
F. speciosa

Sanaclo streptanthilolius

C. leprosepale

Deschampsia caespllosa

Arnica spp.

Pediculeris spp.

Phieum alpinum

Trollis laxus

C. unilliora

Cornus canadensis

V. caespilosum

E. arvanse

C. cenadensis

Luzula perviliora

Equiselum sclipoldes

S. trlangularis

S. amplexilolius

E. eximius

(E. superbus)

Steate et at.

1981, 1983

Devellce and

Ludwig 1983

Ludwig 1979

Steela et al. 1983

co.cllmax will A. lastocarpa

P. monzies"l
P. pungons.

A. concolor

P. strobliosmis

P. remuioldes

Coot mois!

climax.

A. lasiocarpa

minor climax

A. tasiocarpa
P. menzlesii

A. menzlesii
P. pungens

P. ponderosa
P. contorta

Cool dry

P. engelmannil

P. tromuloldes

ctimax

Coot molst

P. ongeimannll A. lasiocarpe

climax $P$ tremuloldes

B. cllarus
G. Hchardso

G. "larizardicus

L. arizonicus
S. srollera

G. trillorum

C. canadens/s
A. rubra

A. rubra

S. siellata

G. rossii

Festuca brachyphylla

P. pulcherrimum

(P. dallcalum)

H. sphondyllum

(H. lenatum)

conicera Involucrara

B. clllere

E. eximius

G. richardsonll

M. clllata

V. canadens/s

H. revolutum

Cladonla llmbriata

Discranowiesla crispula

s. amplexilolius

S. triangularis

Cool dry

ctimax or

P. manzios

to well.
dralned

P. monzlas / (ID)

P. albiceulis

S. bronchialls

J. communis
C. rossil

Davellce et al

$\begin{array}{ll}\text { P. ange/mannll } & \text { A. lasiocarpa } \\ \text { climax. } & \text { P. lramuloldes }\end{array}$

A. lasiocarpa

Cool molst P. engeimann/l A. Jaslocarpa

cllmax or

co.cllmax with P. manzlasi

$\begin{array}{ll}\text { A. laslocarpa } & \text { P. pungons } \\ \text { A. concolor } & \text { P. pondarosa }\end{array}$

$\begin{array}{ll}\text { A. concolor } & \text { P. pondarosa } \\ \text { P. menziosil } & \text { P. strobitorm/s }\end{array}$

$\begin{array}{lll}\text { Cool dry } & P . \text { angelmannll } & \text { P. manzlash } \\ \text { lo woll. } & \text { cllmax } & \text { P. } / \text { laxilis }\end{array}$

lo woll.

dralnod

B. cllialus

F. ovalls

(F. virginiana)

P. aquilinum

S. straptanthilollus

A. communis

A. cordilolia
O. chllansis

O. chilansis
P. sacunda

S. stellate

S. oraophlius

Q. richardsonil

T. occidentala

T. dasyphyllum

Trisolum splcatum

Pyrola minor

Moss spp.

Moss spp.
Rose spp.

Vaccinlum spp. 



\begin{tabular}{|c|c|c|c|c|c|c|}
\hline $\begin{array}{l}\text { Habltat type or } \\
\text { community type }\end{array}$ & $\begin{array}{l}\text { Location and } \\
\text { elevation (10ot) }\end{array}$ & Site & $\begin{array}{l}\text { Successlonal } \\
\text { atatus }\end{array}$ & $\begin{array}{l}\text { Tree } \\
\text { assoclates }\end{array}$ & $\begin{array}{l}\text { Principal undergrowth } \\
\text { species }\end{array}$ & Authority \\
\hline \multicolumn{7}{|c|}{ Abies lasiacarpa series } \\
\hline $\begin{array}{l}\text { Ables laslocarpal } \\
\text { Acar glabrum H.T. } \\
\text { A. glabrum (typlc) phase } \\
\text { Pachistima myrsiniles phase } \\
\text { (ID,WY) }\end{array}$ & $\begin{array}{l}\text { Mountalns of } \\
\text { central and } \\
\text { southern Idaho } \\
(5,000 \cdot 8,000) \text {, } \\
\text { northern and } \\
\text { central Utah, } \\
\text { northwestern } \\
\text { Wyoming }(6,000 . \\
10,000) \text {, and } \\
\text { southwestern } \\
\text { New Mexico } \\
(9,700 \cdot 10,000)\end{array}$ & Warm molst & $\begin{array}{l}\text { A. lastocarpa } \\
\text { climax. } \\
\text { P. angeimannll } \\
\text { may be minor } \\
\text { cllmax }\end{array}$ & $\begin{array}{l}\text { P. engalmannll } \\
\text { P. manzlastl } \\
\text { P. contorta } \\
\text { A. concolor } \\
\text { A. grandls } \\
\text { P. pungans } \\
\text { P. llaxillis } \\
\text { P. tramuloldes }\end{array}$ & $\begin{array}{l}\text { A. glabrum } \\
\text { B. rapans } \\
\text { P. myrsinites } \\
\text { B. clllatus } \\
\text { A. cordllolla } \\
\text { O. chllensls } \\
\text { T. fandlarl } \\
\text { T. occldantale }\end{array}$ & $\begin{array}{l}\text { Alexander el al. } \\
1987 \\
\text { Mauk and } \\
\text { Henderson } 1984 \\
\text { Steele et al. } \\
\text { 1981, } 1883 \\
\text { Youngblood and } \\
\text { Mauk } 1985\end{array}$ \\
\hline $\begin{array}{l}\text { Abies laslocarpal } \\
\text { Alnus sinuata H.T. }\end{array}$ & $\begin{array}{l}\text { Mountains of } \\
\text { northern Montana } \\
\text { and centrat Idaho } \\
(5,000 \cdot 7,500)\end{array}$ & Coot molst & $\begin{array}{l}\text { A, laslocarpa } \\
\text { cllmax }\end{array}$ & $\begin{array}{l}\text { P. angalmannll } \\
\text { P. menzles/l } \\
\text { P. contorla } \\
\text { P. alblcaulis } \\
\text { L. occldentalls }\end{array}$ & $\begin{array}{l}\text { A. sinuata } \\
\text { V. globulare } \\
\text { V. scoparlum } \\
\text { P. secunda } \\
\text { X. tenax }\end{array}$ & $\begin{array}{l}\text { Pflster et al. } 1977 \\
\text { Steele et at. } 1981\end{array}$ \\
\hline $\begin{array}{l}\text { Ables laslocarpal } \\
\text { Berberls repens H.T. } \\
\text { B. repens (lyplc) phase } \\
\text { Plcea engelmannll phase (UT) } \\
\text { Pseudolsuga menzles"l phase (UT) } \\
\text { Plnus flexills phase (UT) } \\
\text { Juniperus communis phase (UT) } \\
\text { Rlbes monligenum phase (UT) } \\
\text { Carex geyerl phase (UT) }\end{array}$ & $\begin{array}{l}\text { Mountains of } \\
\text { Uiah }(6,000 . \\
10,800) \text {, north. } \\
\text { western Wyoming, } \\
\text { and southeastern } \\
\text { (daho } \\
(6,600 \cdot 9,000)\end{array}$ & $\begin{array}{l}\text { Warm to } \\
\text { cool well. } \\
\text { dralned }\end{array}$ & $\begin{array}{l}\text { A. laslocarpa } \\
\text { climax. } \\
\text { P. engelmann/l } \\
\text { minor ctlmax }\end{array}$ & $\begin{array}{l}\text { P. engalmannll } \\
\text { P. contorta } \\
\text { P. pungens } \\
\text { P. menzles/l } \\
\text { A. concolor } \\
\text { A. grandls } \\
\text { P. llexills } \\
\text { P. tremuloldes }\end{array}$ & $\begin{array}{l}\text { B. rapens } \\
\text { J. communis } \\
\text { P. myrsiniles } \\
\text { R. montigenum } \\
\text { R. woodshl } \\
\text { S. oreophlius } \\
\text { C. geyell } \\
\text { C. rossill }\end{array}$ & $\begin{array}{l}\text { Mauk and } \\
\text { Henderson } 1984 \\
\text { Pttster } 1972 \\
\text { Steete el at. } 1983 \\
\text { Youngblood and } \\
\text { Mauk 1985 }\end{array}$ \\
\hline $\begin{array}{l}\text { Ables laslocarpal } \\
\text { Clematls pseudoalpina H.T. }\end{array}$ & $\begin{array}{l}\text { Mountalns of } \\
\text { Montana east } \\
\text { of Continentat } \\
\text { Divide } \\
(6.000-8,000)\end{array}$ & Warm dry & $\begin{array}{l}\text { A. laslocarpa } \\
\text { climax }\end{array}$ & $\begin{array}{l}\text { P. engelmannil } \\
\text { P. menzles/l } \\
\text { P. contorla } \\
\text { P. alb/caulls } \\
\text { P. llexills }\end{array}$ & $\begin{array}{l}\text { C. pseudoalpina } \\
\text { C. tenulloba } \\
\text { A. cordllolla } \\
\text { G. boraale } \\
\text { Valerlans diolce }\end{array}$ & Pfister et al. 1977 \\
\hline $\begin{array}{l}\text { Ables laslocarpal } \\
\text { Holodiscus dumosus H.T. } \\
\text { (Scree foresi) }\end{array}$ & $\begin{array}{l}\text { Mountains of } \\
\text { southwestern } \\
\text { New Mexlco } \\
(9,500 \cdot 10,500)\end{array}$ & Cool dry & $\begin{array}{l}\text { A. leslocarpa } \\
\text { climax or } \\
\text { co.cllmax with } \\
\text { P. slrobltom/s } \\
\text { P. menzles/l. } \\
\text { P. engelmannll } \\
\text { minor climax }\end{array}$ & $\begin{array}{l}\text { P. strobllorm/s } \\
\text { P. menzles/l } \\
\text { P. engelmannll } \\
\text { P. tremuloldes }\end{array}$ & $\begin{array}{l}\text { H. dumosus } \\
\text { J. communis } \\
\text { S. oreophlius } \\
\text { B. clllatus } \\
\text { G. rlchardsonll } \\
\text { H. parryl }\end{array}$ & $\begin{array}{l}\text { Fitzhugh et al. } \\
1987\end{array}$ \\
\hline $\begin{array}{l}\text { Ables laslocarpal } \\
\text { Jamesla americana H.T. } \\
\text { (Scree forest) }\end{array}$ & $\begin{array}{l}\text { Mountatns of } \\
\text { south-central } \\
\text { Arlzona } \\
(8,700-9.100)\end{array}$ & Cool dry & $\begin{array}{l}\text { A. laslocarpa } \\
\text { climax }\end{array}$ & P. menzlesill & $\begin{array}{l}\text { J. americana } \\
\text { Ribes pinetorum } \\
\text { Sambucus melanocarpa } \\
\text { S. oreophllus }\end{array}$ & $\begin{array}{l}\text { Muldavln et al. } \\
1986\end{array}$ \\
\hline $\begin{array}{l}\text { Ables laslocarpal } \\
\text { Junlperus communis H.T. } \\
\text { [A. laslocarpa. } \\
\text { Picea engelmannill } \\
\text { J. communls H.T.] }\end{array}$ & $\begin{array}{l}\text { Mountains of } \\
\text { central Idaho, } \\
\text { northwestern } \\
\text { Wyomtng }(7,500 \text {. } \\
9,500) \text {, Utah, } \\
\text { northern Arizona, } \\
\text { southwestern New } \\
\text { Mextco, and } \\
\text { south-central } \\
\text { Colorado } \\
\text { (8.500-10.500) }\end{array}$ & $\begin{array}{l}\text { Warm to } \\
\text { cold dry }\end{array}$ & $\begin{array}{l}\text { A. laslocarpa } \\
\text { cllmax or } \\
\text { co.climax wlth } \\
\text { P. engelmannll }\end{array}$ & $\begin{array}{l}\text { P. angelmannll } \\
\text { P. menzies"I } \\
\text { P. contorla } \\
\text { A. concolor } \\
\text { P. pungens (UT) } \\
\text { P. longaeva (UT) } \\
\text { P. Iexills } \\
\text { P. tremuloldes }\end{array}$ & $\begin{array}{l}\text { J. communis } \\
\text { A. uva.ursl } \\
\text { A. woodsli } \\
\text { S. canadens/s } \\
\text { S. oreophlius } \\
\text { Poa spp. } \\
\text { A. cordifolla } \\
\text { P. secunda }\end{array}$ & $\begin{array}{l}\text { Komarkova et at. } \\
1988 \\
\text { Mauk and } \\
\text { Henderson } 1984 \\
\text { Molr and } \\
\text { Ludwig } 1979 \\
\text { Steele et al. } \\
\text { 1981, 1983 } \\
\text { Youngblood and } \\
\text { Mauk 1985 }\end{array}$ \\
\hline 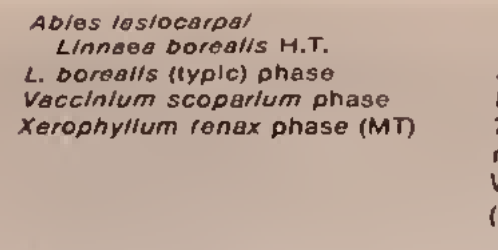 & $\begin{array}{l}\text { Mountalns of } \\
\text { Montana, central } \\
\text { and southeastern } \\
\text { deho }(5,000 \text { - } \\
7,500) \text {, and } \\
\text { northwestern } \\
\text { Wyomlng } \\
(7,000-8,500)\end{array}$ & $\begin{array}{l}\text { Cool molst } \\
\text { to well. } \\
\text { dralned }\end{array}$ & $\begin{array}{l}\text { A. fasiocarpa } \\
\text { climax or or with } \\
\text { co.cllmax with } \\
\text { P. ongelmennll }\end{array}$ & $\begin{array}{l}\text { P. ongolmannll } \\
\text { P. monzlosil } \\
\text { P. contorta } \\
\text { P. ponderosa } \\
\text { P. monlicota } \\
\text { L. occldentalls } \\
\text { P. tremuloldes }\end{array}$ & $\begin{array}{l}\text { L. Doroslls } \\
\text { S. canadensis } \\
\text { v. scoparium } \\
\text { C. rubescens } \\
\text { A. cordliolla } \\
\text { T. occldentells } \\
\text { X. lenax }\end{array}$ & $\begin{array}{l}\text { Pilster et al. } 1977 \\
\text { Steele ot al. } \\
1981,1983\end{array}$ \\
\hline $\begin{array}{l}\text { Ablas laslocarpal } \\
\text { Manzlesla ferruginas H.T. } \\
\text { M. farrugines (Iyplc) phase } \\
\text { Vaccinlum scoparlum phase (ID) } \\
\text { Luzula hilchcockll phase (ID) } \\
\text { Coplls occldentalls phase (ID) } \\
\text { Xerophylfum tenax phase (ID) }\end{array}$ & $\begin{array}{l}\text { Mountatns of } \\
\text { eastern Washington, } \\
\text { Montana, Idaho, } \\
\text { and northwestern } \\
\text { Wyoming } \\
(5,000-7,500)\end{array}$ & Coot molst & $\begin{array}{l}\text { A. lasiocarpa } \\
\text { climax }\end{array}$ & $\begin{array}{l}\text { P. angelmannll } \\
\text { P. menzleshl } \\
\text { P. contorta } \\
\text { P. montlcola } \\
\text { P. albicaulls } \\
\text { L. occldentalls } \\
\text { T. mertenstana }\end{array}$ & $\begin{array}{l}\text { M. lerruginea } \\
\text { Ledum glandulosum } \\
\text { Rhododendron alblllorum } \\
\text { V. globulare } \\
\text { V. scoparlum } \\
\text { L. hlchcockll } \\
\text { C. occldentalls } \\
\text { X. lenax }\end{array}$ & $\begin{array}{l}\text { Cooper el at. } 1987 \\
\text { Daubenmlre and } \\
\text { Daubenmlre } 1968 \\
\text { Pflster et at. } 1977 \\
\text { Steele et al. } \\
\text { 1981, } 1983\end{array}$ \\
\hline $\begin{array}{l}\text { Ables laslocarpal } \\
\text { Oplopanax horrldum H.T. }\end{array}$ & $\begin{array}{l}\text { Mountains of } \\
\text { northern Montana } \\
(4,000 \cdot 5,000)\end{array}$ & $\begin{array}{l}\text { Cooi molst } \\
\text { wet }\end{array}$ & $\begin{array}{l}\text { A. laslocarpa } \\
\text { co.climax wifh } \\
\text { P. ongelmannll }\end{array}$ & $\begin{array}{l}\text { P. engelmannll } \\
\text { P. menzlosil } \\
\text { P. monticola } \\
\text { L. occldentalls }\end{array}$ & $\begin{array}{l}\text { O. horridum } \\
\text { T. bravilolla } \\
\text { C. unillora } \\
\text { Tlaralla lrilollata }\end{array}$ & Ptlster el al. 1977 \\
\hline $\begin{array}{l}\text { Ables laslocarpal } \\
\text { Pachlstima myrsinlios H.T. } \\
\text { A. laslocarpa. } \\
\text { Picea angalmannill' } \\
\text { P. myrsinites H.T.(CO)] }\end{array}$ & $\begin{array}{l}\text { Mountalns of } \\
\text { northern Idaho and } \\
\text { eastern Washington } \\
(4,700-5,800) \text {, and } \\
\text { central Colorado } \\
(9,000 \cdot 9,500)\end{array}$ & $\begin{array}{l}\text { Cool to } \\
\text { weff. } \\
\text { drained }\end{array}$ & $\begin{array}{l}\text { A. laslocarpa } \\
\text { cilmax or } \\
\text { co.cllmax wilh } \\
\text { P. angelmannll }\end{array}$ & $\begin{array}{l}\text { P. engelmannll } \\
\text { P. menzles/l } \\
\text { P. contorta } \\
\text { P. monticola } \\
\text { L. occldentalls } \\
\text { P. tremuloldes }\end{array}$ & $\begin{array}{l}\text { P. myrsiniles } \\
\text { V. scoparium } \\
\text { C. geyerl } \\
\text { A. cordllolle } \\
\text { C. unilfora } \\
\text { Erigeron spp. } \\
\text { G. Hillorum }\end{array}$ & $\begin{array}{l}\text { Daubenmire and } \\
\text { Daubenmire } 1968 \\
\text { Hess and Wasser } \\
1982\end{array}$ \\
\hline $\begin{array}{l}\text { Ables laslocarpal } \\
\text { Physocarpus malvaceus H.T. }\end{array}$ & $\begin{array}{l}\text { Mountains of } \\
\text { southeastern Idaho, } \\
\text { northwestern } \\
\text { Wyomlng, and } \\
\text { northern and } \\
\text { centrat Utah } \\
(6,000-9,500)\end{array}$ & $\begin{array}{l}\text { Warm molst } \\
10 \text { well. } \\
\text { dralned }\end{array}$ & $\begin{array}{l}\text { A. laslocerpe } \\
\text { climax }\end{array}$ & $\begin{array}{l}\text { P. ongolmennil } \\
\text { P. monzles"l } \\
\text { A. concolor } \\
\text { A. grandis } \\
\text { P. tramuloldes }\end{array}$ & $\begin{array}{l}\text { P. malvacaus } \\
\text { A. alnifolla } \\
\text { S. betullfolla } \\
\text { Sorbus scopulina } \\
\text { S. albus }\end{array}$ & $\begin{array}{l}\text { Mauk and } \\
\text { Henderson } 1984 \\
\text { Steele el al. } 1983 \\
\text { Youngblood and } \\
\text { Mauk } 1985\end{array}$ \\
\hline $\begin{array}{l}\text { Ables laslocarpal } \\
\text { Ribes moniliganum H.T. } \\
\text { A. monilgenum (typic) phase } \\
\text { Pinus albicaulls phase (ID,WY) } \\
\text { Pinus contoria phase (UT) } \\
\text { Trisalum splcalum phase (UT) } \\
\text { Mertensla erlzonice phase (UT) } \\
\text { Thallcirum fondlerl phase (UT) }\end{array}$ & $\begin{array}{l}\text { Mountatns of } \\
\text { southern Montana, } \\
\text { Idaho, Utah, } \\
\text { and northwestern } \\
\text { Wyoming } \\
(8,000-11,000)\end{array}$ & Cool dry & $\begin{array}{l}\text { A. laslocerpa } \\
\text { cllmax or } \\
\text { co.cllmax with } \\
\text { P. engolmannll }\end{array}$ & $\begin{array}{l}\text { P. ongalmannil } \\
\text { P. menzlasil } \\
\text { P. contorla } \\
\text { P. albicaulls } \\
\text { P. tromuloides }\end{array}$ & $\begin{array}{l}\text { R. montigenum } \\
\text { T. splcalum } \\
\text { C. rossill } \\
\text { A. microphylla } \\
\text { A. tatilolla } \\
\text { M. arlzonica } \\
\text { O. chllensis } \\
\text { T. tendlarl }\end{array}$ & $\begin{array}{l}\text { Mauk and } \\
\text { Henderson } 1984 \\
\text { Pllster } 1972 \\
\text { Ptlster et al. } 1977 \\
\text { Steele et al. } \\
\text { 1981, 1983 } \\
\text { Youngblood and } \\
\text { Mauk 1985 }\end{array}$ \\
\hline $\begin{array}{l}\text { Ables laslocarpal } \\
\text { Rubus parvillorus H.T. }\end{array}$ & $\begin{array}{l}\text { Mountatns of } \\
\text { northern and south. } \\
\text { western New Mexlco, } \\
\text { and southwestern } \\
\text { Colorado } \\
(8,500 \cdot 10,500)\end{array}$ & Warm moist & $\begin{array}{l}\text { A. laslocarpa } \\
\text { co.cllmax with } \\
\text { P. engalmannil }\end{array}$ & $\begin{array}{l}\text { P. engelmannll } \\
\text { P. menzlas/l } \\
\text { A. concolor } \\
\text { P. strobilomis } \\
\text { P. tremuloldes }\end{array}$ & $\begin{array}{l}\text { A. parvillorus } \\
\text { A. glabrum } \\
\text { P. myrsinltes } \\
\text { V. myrthlus } \\
\text { B. clllefus } \\
\text { E. eximius } \\
\text { (E. suparbus) } \\
\text { G. richerdsonll }\end{array}$ & $\begin{array}{l}\text { Devellce et at. } \\
1986 \\
\text { Fltzhugh et al. } \\
1987 \\
\text { Moir and } \\
\text { Ludw/g } 1979\end{array}$ \\
\hline $\begin{array}{l}\text { Ables lasiocarpal } \\
\text { Sellx glauca H.T. } \\
\text { [A. laslocerpa. } \\
\text { Piceo angelmannill } \\
\text { S. glauce H.T.] }\end{array}$ & $\begin{array}{l}\text { High mountains } \\
\text { of south-centsal } \\
\text { Colorado } \\
(11,000 \cdot 11,800)\end{array}$ & Cold wet & $\begin{array}{l}\text { A. lasiocarpa } \\
\text { co.climax wilth } \\
\text { P. engermannll }\end{array}$ & P. ongelmennll & $\begin{array}{l}\text { S. glauce } \\
\text { V. myrillius } \\
\text { Carex spp. } \\
\text { Acomastylis rossil } \\
\text { P. pulcherrimum } \\
\text { (P. delicatum) }\end{array}$ & $\begin{array}{l}\text { Komarkova et al. } \\
1988\end{array}$ \\
\hline $\begin{array}{l}\text { Ables lasiocarpal } \\
\text { Shopherdia canadensis H.T. }\end{array}$ & $\begin{array}{l}\text { Blghorn Mountalns, } \\
\text { north.central } \\
\text { Wyoming } \\
(8,000-8,500)\end{array}$ & Warm dry & $\begin{array}{l}\text { A. leslocarpa } \\
\text { co.climax with } \\
\text { P. ongeimannll }\end{array}$ & $\begin{array}{l}\text { P. engelmennll } \\
\text { P. menzles ll } \\
\text { P. contorta }\end{array}$ & $\begin{array}{l}\text { S. canadensis } \\
\text { B. repens } \\
\text { S. belulliolla } \\
\text { V. scoperlum }\end{array}$ & $\begin{array}{l}\text { Hotfman and } \\
\text { Alexander } 1976\end{array}$ \\
\hline
\end{tabular}





\begin{tabular}{|c|c|c|c|c|c|}
\hline $\begin{array}{l}\text { Habltat typa or } \\
\text { community typo }\end{array}$ & $\begin{array}{l}\text { Location and } \\
\text { elavation (teet) }\end{array}$ & Slte & $\begin{array}{l}\text { Succassionat } \\
\text { status }\end{array}$ & $\begin{array}{c}\text { Trae } \\
\text { assoclates }\end{array}$ & $\begin{array}{l}\text { Principal undargrowth } \\
\text { spectas }\end{array}$ \\
\hline $\begin{array}{l}\text { Ables laslocarpal } \\
\text { Splraea betulliolla H.T. }\end{array}$ & $\begin{array}{l}\text { Mountains of } \\
\text { cantral and } \\
\text { southeastern Idaho, } \\
\text { and northwastern } \\
\text { Wyoming } \\
(5,500-7,500)\end{array}$ & Warm dry & $\begin{array}{l}\text { A, lasiocarpa } \\
\text { cllmax }\end{array}$ & $\begin{array}{l}\text { P. engelmannil } \\
\text { P. menzlesil } \\
\text { P. contoria }\end{array}$ & $\begin{array}{l}\text { S. beluillolla } \\
\text { A. ainllolla } \\
\text { B. rapens } \\
\text { P. myrsinltas } \\
\text { C. rubascens }\end{array}$ \\
\hline $\begin{array}{l}\text { Ablas lasiocarpal } \\
\text { Symphoricarpos albus H.T. }\end{array}$ & $\begin{array}{l}\text { Mountalns of } \\
\text { southeastern } \\
\text { Id aho and north. } \\
\text { western Wyoming } \\
(5,700 \cdot 7,600)\end{array}$ & $\begin{array}{l}\text { Warm well- } \\
\text { dralned }\end{array}$ & $\begin{array}{l}\text { A. lasiocarpa } \\
\text { climax }\end{array}$ & $\begin{array}{l}\text { P. angelmannil } \\
\text { P. menzlesil } \\
\text { P. contorla } \\
\text { P. tramuloldes }\end{array}$ & $\begin{array}{l}\text { S. arbus } \\
\text { A. ainllolla } \\
\text { C. rubescens } \\
\text { A. cordilolla }\end{array}$ \\
\hline $\begin{array}{l}\text { Ables laslocarpal } \\
\text { Vaccinium caespllosum H.T. } \\
\text { V. caespltosum (typlc) phase } \\
\text { Picea engelmannll phase (UT) }\end{array}$ & $\begin{array}{l}\text { Mountalns of } \\
\text { south-central } \\
\text { Montana, } \\
\text { ldaho }(5,000 \cdot 7,500) \text {, } \\
\text { and northern } \\
\text { and central Utah } \\
(8,500 \cdot 10,000)\end{array}$ & $\begin{array}{l}\text { Cool molst } \\
\text { to wall. } \\
\text { drainad }\end{array}$ & $\begin{array}{l}\text { A. laslocarpa } \\
\text { cllmax. } \\
\text { P. engeimannll } \\
\text { may be minor } \\
\text { cllmax }\end{array}$ & $\begin{array}{l}\text { P. engelmannll } \\
\text { P. menzlesil } \\
\text { P. contoria } \\
\text { P. albicaulls } \\
\text { P. tremuloides }\end{array}$ & $\begin{array}{l}\text { V. caespliosum } \\
\text { L. borealls } \\
\text { P. myrsinltes } \\
\text { Ribas spp. } \\
\text { V. scoparlum } \\
\text { C. rubescens } \\
\text { A. cordllolla }\end{array}$ \\
\hline $\begin{array}{l}\text { Ables lasiocarpal } \\
\text { Vaccinium globulare H.T. } \\
\text { V. globulare (typlc) phase } \\
\text { Pachistima myrsinlies phase } \\
\text { (ID,WY) } \\
\text { Vaccinlum scoparium phase } \\
\text { (ID,WY) }\end{array}$ & $\begin{array}{l}\text { Mountalns of } \\
\text { south.central } \\
\text { Montana and } \\
\text { Idaho }(5,000 \cdot \\
8,700) \text {, northern } \\
\text { Utah, and norlh. } \\
\text { western Wyoming } \\
(7,000 \cdot 9,500)\end{array}$ & $\begin{array}{l}\text { Cool molst } \\
\text { to well. } \\
\text { drained }\end{array}$ & $\begin{array}{l}\text { A. laslocarpa } \\
\text { cllmax }\end{array}$ & $\begin{array}{l}\text { P. engelmannil } \\
\text { P. menzlesil } \\
\text { P. contorta } \\
\text { P. alblcaulls }\end{array}$ & $\begin{array}{l}\text { V. globulare } \\
\text { L. utahansis } \\
\text { P. myrsinites } \\
\text { R. monilgenum } \\
\text { S. oreophllus } \\
\text { V. scoparlum }\end{array}$ \\
\hline 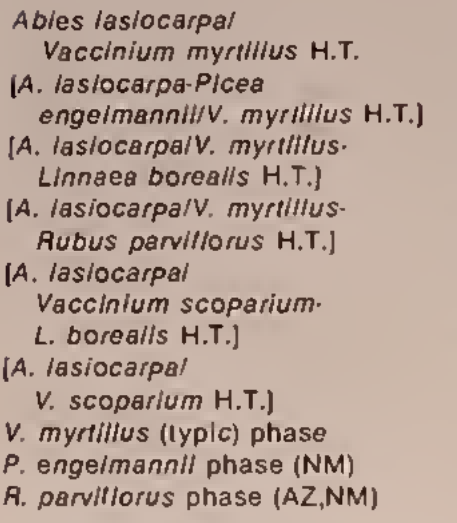 & $\begin{array}{l}\text { Mounlains ol } \\
\text { easlem and } \\
\text { south.central } \\
\text { Arlzona, northarn } \\
\text { and southwestern } \\
\text { New Mexlco, southern } \\
\text { and western } \\
\text { Colorado }(9,000 . \\
11,000) \text {, and } \\
\text { cantral Utah } \\
(10,000 \cdot 10.600)\end{array}$ & $\begin{array}{l}\text { Cool dry } \\
\text { to well. } \\
\text { dralned }\end{array}$ & $\begin{array}{l}\text { A. laslocarpa } \\
\text { cllmax or } \\
\text { co.cllmax with } \\
\text { P. engelmannil }\end{array}$ & $\begin{array}{l}\text { P. engalmannll } \\
\text { P. menzlesll } \\
\text { A. concolor } \\
\text { P. pungens } \\
\text { P. contorla (CO) } \\
\text { P. strobllormis } \\
\text { P. llexllis } \\
\text { P. arlstala } \\
\text { P. tremuloldes }\end{array}$ & $\begin{array}{l}\text { V. myrtllus } \\
\text { L. borealls } \\
\text { P. myrsinites } \\
\text { Ramischla secunta } \\
\text { R. monilgenum } \\
\text { R. parviliorus } \\
\text { V. scoparlum } \\
\text { B. clllatus } \\
\text { E. eximius } \\
\text { (E. superbus) } \\
\text { O. chllensis } \\
\text { P. racemosa }\end{array}$ \\
\hline $\begin{array}{l}\text { Ables lasiocarpal } \\
\text { Vaccinlum scoparium H.T. } \\
\text { A. laslocarpa. } \\
\text { Plcea engelmannill } \\
\text { V. scoparlum H.T. } \\
\text { (P. engelmannill } \\
\text { V. scoparium H.T.) } \\
\text { V. scoparlum (Iypic) phase } \\
\text { Pinus albicaulls phase (ID,Wn } \\
\text { Catamagrostis rubescens phase } \\
\text { (10,MT.Wn }\end{array}$ & $\begin{array}{l}\text { Mountains of } \\
\text { Montana and } \\
\text { Idaho }(5,000- \\
10,000) \text { soulh to } \\
\text { Arizona and } \\
\text { New Mexlco } \\
(8,000 \cdot 11,000)\end{array}$ & Cool dry & $\begin{array}{l}\text { A. laslocarpa } \\
\text { climax or } \\
\text { co.cllmax wlth } \\
\text { P. engelmannil }\end{array}$ & $\begin{array}{l}\text { P. angeimannll } \\
\text { P. menziesil } \\
\text { P. contorta } \\
\text { P. albicaulls } \\
\text { L. occidantalls } \\
\text { P. tremuloides }\end{array}$ & $\begin{array}{l}\text { V. scoparium } \\
\text { L. borealls } \\
\text { P. myrsinites } \\
\text { V. myrllilus } \\
\text { C. rubescens } \\
\text { C. geyerl } \\
\text { A. cordilolla } \\
\text { A. talliolla } \\
\text { E. eximius } \\
\text { (E. Superbus) } \\
\text { Phyllodoco emporriform/s }\end{array}$ \\
\hline
\end{tabular}

Staale at al.

1981,1983

Cooper ot al. 1987 Mauk and

enderson 1984

Fister et al. 1977

Youngblood and

Mauk 1985

Cooper et al. 1987

Henderson 1984

Steele el al.
1981,1983

Youngblood and
Mauk 1985

Alexander et al.

1987

DeVetice

1986

Devetlce and

Ludwig 1983
Fitzhugh el al.

1987

Holfman 1988

omarkova et al.

1988
Molr and

Ludwlg 1979

Youngblood and

Alexander et al.

1986

Cooper et al. 1987

Daubenmire 1968 Hess and
Hex

Alexander 1986

1982

Hoftman 1988

Alexander 197

1980. 1983 Komarkova el al.

1988

Henderson 198

Mols and Ludwig

1979

Pilster el al. 1977

Steele et al.

ister el ai. 1977

V. scoparium

C. rossill

$x$. tenax

Hieracium graclle

Vaccinlum scoparium H.T. $\quad$ ol Con

$\begin{array}{ll}\text { co.cllmax with } & \text { P. engelmannil } \\ P \text {. albicaulls } & \text { P. conlorta }\end{array}$

C. canadensis

Utah, and north

and central

$(8,000 \cdot 10,500)$

C. canadensis (typlc) phase
Vaccinium caespltosum phas$$
\text { (ID,MT }
$$

Gallum trillorum phase (MT)

Ledum g/andulosum phase (ID)

Ledum glandulosum phase (ID)

Ables laslocarpal

Calamagrosils ruboscens H.T

C. rubascens (lyplc) phase

chistima myrsinites phase$$
\text { (ID,WY }
$$

Mounlains of

Montana easi

ol Conlinental

Divide, Idaho,

northern Ulah,

Wyoming

Mountains of

Abies laslocarpal

Mountalns ol

Monlana west

ol Conlinental

Divide, Idaho,

L. hilchcockil (lyplc) phase

Manziasla lerruginea phase (MT)

(ID,MT)

Ables laslocarpal

Carex gayorl H.T.

A. laslocarpa.

Plcea engalmannill

C. geyeri H.T.

C. geyarl (typlc) phase

Pseudorsuga menziasil phase
(ID,MT)

Artemisla tridantata phase (ID)
$(6,000-8,000)$

Mounlains of

cantral Montana,

central ldaho,

southern Utah

$(6,500 \cdot 9,500)$.

wyoming, and

Colorado $(8,500 \cdot 11,000)$
L. glandulosum

Vaccinum spp.

Carex spp.

$E$. arvense

G. Iriflorum

L. canby

S. Iriangularis colimax or

$P$. engelmannil

$P$. pungens (UT)
$P$. albicaulls

P. Iremuloldes
Hess and

Alexander 1986 Komarkova el al. 1988

Mauk and

Henderson 1984

Pllster el al. 1977

1981,1983

Cooper et al. 1987 Mauk and

Handerson 198 Plistar at al. 197

1981,1983

P. myrsinites

C. geyarl

A. cordilolla

O. chllansis
T. occidantale

Viola adunca

L. hllchcock"l

M. lerruginea

V. scoparium

A. cordillille

$x$. Lenox

Larix lyalli!

P. contorta

P. albicaulis

L. lyalli (MT)

Cool dry

$\begin{array}{lll}\text { A. lasiocarpa } & \text { P. engelmannII } & \text { C. geyerl } \\ \text { climax or } & \text { P. manzles } /(M T, I D) & \text { A. tridantate }\end{array}$

climax or climax with P. contorta

A. woodsil

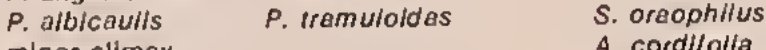

Alaxander 1986
Hass and Wasser

Lathyrus lanszweril

L. argentaus

O. chilensis
S. stellata
Cooper al al. 1987 Pister at al. 1977
Steala at al.

1981, 1983

Alexander at al.

1986 1982

Hollman 1988

Hollman and

Alaxander 1976,

1983

Komarkova al al.

1988

Pllstar et al. 1977

Steele et al.

1981,1983

Youngblood and

Mauk 1985 



\begin{tabular}{|c|c|c|c|c|c|c|}
\hline $\begin{array}{l}\text { Habltat type or } \\
\text { community type }\end{array}$ & $\begin{array}{l}\text { Location and } \\
\text { elevation (faet) }\end{array}$ & Site & $\begin{array}{l}\text { Successlonel } \\
\text { status }\end{array}$ & $\begin{array}{c}\text { Trea } \\
\text { assocletes }\end{array}$ & $\begin{array}{l}\text { Principal undergrowth } \\
\text { speclas }\end{array}$ & Authority \\
\hline $\begin{array}{l}\text { Ables laslocarpal } \\
\text { Carex rossil H.T. }\end{array}$ & $\begin{array}{l}\text { Mountains of } \\
\text { southaastern Ideho, } \\
\text { northwestern } \\
\text { Wyoming }(7,500 \text {. } \\
8,000) \text {, and } \\
\text { central and } \\
\text { southern Utah } \\
(8,500-10,500)\end{array}$ & Cool dry & $\begin{array}{l}\text { A. lasiocarpa } \\
\text { cllmax }\end{array}$ & $\begin{array}{l}\text { P. angalmannll } \\
\text { P. manzlasil } \\
\text { P. contorta } \\
\text { P. Ilaxills } \\
\text { P. tramuloldes }\end{array}$ & $\begin{array}{l}\text { C. rossil } \\
\text { A. palula } \\
\text { Albas viscosissimum } \\
\text { A. cordllolla } \\
\text { A. angelmannil } \\
\text { A. misar }\end{array}$ & $\begin{array}{l}\text { Steele el al. } 1983 \\
\text { Youngblood and } \\
\text { Mauk } 1985\end{array}$ \\
\hline $\begin{array}{l}\text { Ables laslocarpal } \\
\text { Aconilum columbianum H.T. }\end{array}$ & $\begin{array}{l}\text { Mountelns of } \\
\text { cantral and } \\
\text { southern Utah } \\
(7,400-10,000)\end{array}$ & Cool molst & $\begin{array}{l}\text { A. laslocarpa } \\
\text { cllmax. } \\
\text { P. engaimannll } \\
\text { minor cllmax }\end{array}$ & $\begin{array}{l}\text { P. engalmannil } \\
\text { P. manzlasil } \\
\text { A. concolor } \\
\text { P. tramuioldas }\end{array}$ & $\begin{array}{l}\text { A. columblanum } \\
\text { A. montlgenum } \\
\text { B. clllatus } \\
\text { A. rubra } \\
\text { A. cordllolla } \\
\text { G. richardsonll } \\
\text { O. chllansls }\end{array}$ & $\begin{array}{l}\text { Youngblood and } \\
\text { Mauk } 1985\end{array}$ \\
\hline $\begin{array}{l}\text { Ables laslocarpal } \\
\text { Actaea rubra H.T. }\end{array}$ & $\begin{array}{l}\text { Mountalns of } \\
\text { cantral Idaho, } \\
\text { northern Utah, } \\
\text { and northwestarn } \\
\text { Wyoming } \\
(6,000-8,000)\end{array}$ & Warm molst & $\begin{array}{l}\text { A. laslocarpa } \\
\text { co.cllmax with } \\
\text { P. angelmannll }\end{array}$ & $\begin{array}{l}\text { P. angalmannll } \\
\text { P. pungens } \\
\text { P. manzlasll } \\
\text { P. contorta } \\
\text { A. concolor } \\
\text { A. grandls } \\
\text { P. tramuloldas }\end{array}$ & $\begin{array}{l}\text { A. rubra } \\
\text { 8. repans } \\
\text { L. ulahensis } \\
\text { R. parvillorus } \\
\text { V. giobulara } \\
\text { O. chilensls } \\
\text { T. landlarl }\end{array}$ & $\begin{array}{l}\text { Mauk and } \\
\text { Henderson } 1984 \\
\text { Steale el al. } 1983\end{array}$ \\
\hline 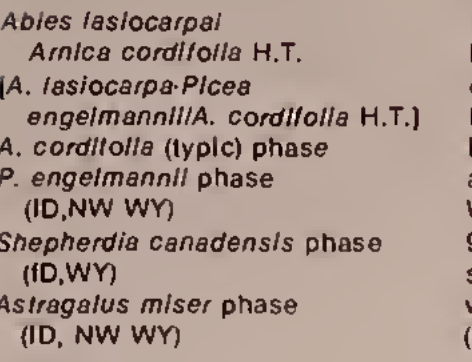 & $\begin{array}{l}\text { Mountains of } \\
\text { Montana east } \\
\text { of Continental } \\
\text { Dlvide, cantral } \\
\text { Idaho, northwestern } \\
\text { and north.central } \\
\text { Wyoming }(7,000 \text {. } \\
9,500) \text {, and } \\
\text { south-central and } \\
\text { western Colorado } \\
(9,000 \cdot 11,000)\end{array}$ & $\begin{array}{l}\text { Cool dry } \\
\text { to well. } \\
\text { drained }\end{array}$ & $\begin{array}{l}\text { A. laslocarpa } \\
\text { climax or } \\
\text { co-climax with } \\
\text { P. angelmannil }\end{array}$ & $\begin{array}{l}\text { P. engelmannill } \\
\text { P. manzlesll } \\
\text { P. contorta } \\
\text { P. albicaulls } \\
\text { P. llaxills } \\
\text { P. Hemuloldes }\end{array}$ & $\begin{array}{l}\text { A. cordilolla } \\
\text { S. canadensls } \\
\text { A. misar } \\
\text { E. angustllollum } \\
\text { F. ovalls } \\
\text { (F. virginiana) } \\
\text { P. secunda }\end{array}$ & $\begin{array}{l}\text { Hoftman and } \\
\text { Alexander } 1976 \\
\text { Komarkova et al. } \\
1988 \\
\text { Pflster et at. } 1977 \\
\text { Staele el al. } \\
\text { 1981, } 1983\end{array}$ \\
\hline $\begin{array}{l}\text { Ables lasiocarpal } \\
\text { Arnica latllolla H.T. }\end{array}$ & $\begin{array}{l}\text { Mountains of } \\
\text { southeastarn Idaho, } \\
\text { northern Utah, } \\
\text { and northwesiern } \\
\text { Wyoming } \\
(7,400-9,300)\end{array}$ & Coot dry & $\begin{array}{l}\text { A. taslocarpa } \\
\text { cllmax }\end{array}$ & $\begin{array}{l}\text { P. engalmannil } \\
\text { P. menzlesill } \\
\text { P. contorta } \\
\text { P. albicaulls } \\
\text { P. Iremuloldes }\end{array}$ & $\begin{array}{l}\text { A. latilolla } \\
\text { P. myrsiniles } \\
\text { P. monligenum } \\
\text { A. engelmannill } \\
\text { P. racemosa } \\
\text { P. secunda }\end{array}$ & Steela at al. 1983 \\
\hline $\begin{array}{l}\text { Ioles laslocarpal } \\
\text { Caltha blllora H.T. }\end{array}$ & $\begin{array}{l}\text { Mountalns ol } \\
\text { ceniral Idaho } \\
(6,200 \cdot 7,800)\end{array}$ & Cool wet & $\begin{array}{l}\text { A. lasiocarpa } \\
\text { climax }\end{array}$ & $\begin{array}{l}\text { P. engelmannll } \\
\text { P. contorta }\end{array}$ & $\begin{array}{l}\text { C. blllora } \\
\text { L. Involucrata } \\
\text { Dodecaniheon jelfreyl } \\
\text { Pedicularl's bracleosa } \\
\text { S. Iriangularls }\end{array}$ & Steele et al. 1981 \\
\hline $\begin{array}{l}\text { Doles lasiocarpal } \\
\text { Clintonia unlliora H.T. } \\
\text {. Unillora (Iyplc) phase } \\
\text { Menzlesia terruginea phasa } \\
\text { Vaccinlum caespllosum phase } \\
\text { (MT) } \\
\text { Aralla nudicaults phase (Mn } \\
\text { Xerophyllum renax phase }\end{array}$ & $\begin{array}{l}\text { Mountains of } \\
\text { northweslern } \\
\text { Montana, and } \\
\text { northern and } \\
\text { central Idaho } \\
(3,500-6,000)\end{array}$ & $\begin{array}{l}\text { Warm molst } \\
\text { to well. } \\
\text { dralned }\end{array}$ & $\begin{array}{l}\text { A. laslocarpa } \\
\text { cllmax. } \\
\text { Mlnor cllmaxes } \\
\text { A. grandls } \\
\text { T. martensiana }\end{array}$ & $\begin{array}{l}\text { A. grandis } \\
\text { T. mertienslana } \\
\text { P. engalmannll } \\
\text { P. manzlasll } \\
\text { P. contorta } \\
\text { P. monticola } \\
\text { P. ponderosa } \\
\text { L. occldenralls }\end{array}$ & $\begin{array}{l}\text { C. unlliora } \\
\text { M. Iarruginea } \\
\text { P. myrsinites } \\
\text { V. caespllosum } \\
\text { V. globulare } \\
\text { A. nudicaulls } \\
\text { C. occldentalls } \\
\text { X. renax }\end{array}$ & $\begin{array}{l}\text { Cooper el al. } 1987 \\
\text { Pflster et al. } 1977 \\
\text { Steele el al. } 1981\end{array}$ \\
\hline $\begin{array}{l}\text { Ables lasiocarpal } \\
\text { Coporls occidentalls H.T. }\end{array}$ & $\begin{array}{l}\text { Mountains of } \\
\text { central toaho } \\
(5.100-6.700)\end{array}$ & $\begin{array}{l}\text { Warm lo } \\
\text { well. } \\
\text { dralned }\end{array}$ & $\begin{array}{l}\text { A. fastocarpa } \\
\text { climax }\end{array}$ & $\begin{array}{l}\text { P. engelmannil } \\
\text { P. menzlesil } \\
\text { P. contorta } \\
\text { L. occldentalls }\end{array}$ & $\begin{array}{l}\text { C. occidentalls } \\
\text { M. terrugineas } \\
\text { V. globuiare } \\
\text { Anemona plpent } \\
\text { X. tenex }\end{array}$ & Steele et al. 1981 \\
\hline $\begin{array}{l}\text { Ables laslocarpal } \\
\text { Erlgaron eximlus } \\
\text { (E. superbus) H.T. }\end{array}$ & $\begin{array}{l}\text { Mountalns of } \\
\text { southweslern } \\
\text { Colorado, northern } \\
\text { and soulhwestern } \\
\text { New Mexico, and } \\
\text { eastern Arlzona } \\
(9,000-11,000)\end{array}$ & $\begin{array}{l}\text { Cool molst } \\
\text { ro well. } \\
\text { drainad }\end{array}$ & $\begin{array}{l}\text { A. leslocarpa } \\
\text { co.cllmax wilh } \\
\text { P. angalmannll }\end{array}$ & $\begin{array}{l}\text { P. engalmannil } \\
\text { P. manzlesil } \\
\text { A. concolor } \\
\text { P. pungans } \\
\text { P. llaxllis } \\
\text { P. strobllormis } \\
\text { P. Iramuloldes }\end{array}$ & $\begin{array}{l}\text { E. eximius } \\
\text { (E. superbus) } \\
\text { 8. repens } \\
\text { L. involucrata } \\
\text { A. cordilolla } \\
\text { G. richardsonll } \\
\text { L. arizonicus }\end{array}$ & $\begin{array}{l}\text { Alexander at at. } \\
1987 \\
\text { DeVellce el at. } \\
1986 \\
\text { Flizhugh at at. } \\
1987 \\
\text { Moir and } \\
\text { Ludwig } 1979\end{array}$ \\
\hline $\begin{array}{l}\text { bles lasiocarpal } \\
\text { Gallum trillorum H.T. }\end{array}$ & $\begin{array}{l}\text { Mountalns of } \\
\text { northern Montana } \\
(5,000-7,700)\end{array}$ & Werm molst & $\begin{array}{l}\text { A. lasiocarpa } \\
\text { climex }\end{array}$ & $\begin{array}{l}\text { P. angalmannll } \\
\text { P. menziosil } \\
\text { P. contorta } \\
\text { L. occldantalls }\end{array}$ & $\begin{array}{l}\text { G. trillorum } \\
\text { A. rubra } \\
\text { S. trlangularis } \\
\text { S. amplexilolius }\end{array}$ & Pflster et el. 1977 \\
\hline $\begin{array}{l}\text { bles laslocarpal } \\
\text { Lathyrus arizonicus H.T. }\end{array}$ & $\begin{array}{l}\text { Mountalns of } \\
\text { north-central } \\
\text { Arlzona and } \\
\text { southwestern } \\
\text { Now Mexico } \\
(9,500 \cdot 10,500)\end{array}$ & Cool dry & $\begin{array}{l}\text { A. laslocarpa } \\
\text { cllmex or } \\
\text { co-climax wlith } \\
\text { P. ongelmannll }\end{array}$ & $\begin{array}{l}\text { P. engalmannll } \\
\text { P. manzlesil } \\
\text { P. strobllormis } \\
\text { P. Iramuloldas }\end{array}$ & $\begin{array}{l}\text { L. arizonicus } \\
\text { A. glabrum } \\
\text { S. oraophllus } \\
\text { B. cillalus } \\
\text { G. fichardsonll } \\
\text { S. stallata } \\
\text { V. amaricana }\end{array}$ & $\begin{array}{l}\text { Flizhugh el al. } \\
1987 \\
\text { Molr and } \\
\text { Ludwig } 1979\end{array}$ \\
\hline $\begin{array}{l}\text { Ables laslocarpal } \\
\text { Martensia clllata H.T. }\end{array}$ & $\begin{array}{l}\text { Mountains of } \\
\text { northain Naw } \\
\text { Maxico and } \\
\text { southern Colorado } \\
(9,200 \cdot 11,200)\end{array}$ & Cool molst & $\begin{array}{l}\text { A. lasiocarpa } \\
\text { co.cllmex wilh } \\
\text { P. engolmannll }\end{array}$ & $\begin{array}{l}\text { P. angolmannll } \\
\text { P. tramuloldes }\end{array}$ & $\begin{array}{l}\text { M. clliata } \\
\text { Carox balla } \\
\text { C. laptosapala } \\
\text { Cardamine cordllolla } \\
\text { M. pantandra } \\
\text { O. Tandlent }\end{array}$ & $\begin{array}{l}\text { Davellce et al. } \\
\quad 1986\end{array}$ \\
\hline $\begin{array}{l}\text { Ablas lasiocarpal } \\
\text { Osmorhiza chitansis H.T. } \\
\text { o. chllans/s (typlc) phasa } \\
\text { Pachislima myrsinites phasa (ID) }\end{array}$ & $\begin{array}{l}\text { Mounlalns of } \\
\text { soulhaastarn } \\
\text { Idaho and } \\
\text { northarn Ulah } \\
(6,500-8,800)\end{array}$ & $\begin{array}{l}\text { Warm molst } \\
\text { to well. } \\
\text { dralnad }\end{array}$ & $\begin{array}{l}\text { A. lastocarpa } \\
\text { climax }\end{array}$ & $\begin{array}{l}\text { P. angelmannll } \\
\text { P. manzlas"l } \\
\text { P. conforla } \\
\text { P. llaxills } \\
\text { P. alblcaulls } \\
\text { P. tramuloldes }\end{array}$ & $\begin{array}{l}\text { O. chllansis } \\
\text { 8. repans } \\
\text { P. myrsinltes } \\
\text { C. rossll } \\
\text { O. dapapurata } \\
\text { T. tandleri }\end{array}$ & $\begin{array}{l}\text { Meuk and } \\
\text { Henderson } 1984 \\
\text { Steale el al. } 1983\end{array}$ \\
\hline $\begin{array}{l}\text { Ablas laslocarpal } \\
\text { Pedicularis racemosa H.T. } \\
\text { P. racemosa (typlc) phase } \\
\text { Psaudolsuga manzlasill phase (UT) }\end{array}$ & $\begin{array}{l}\text { Mountalns of } \\
\text { southeastern } \\
\text { Idaho, norlh. } \\
\text { westarn Wyoming, } \\
\text { and norlhern } \\
\text { Utah } \\
(7,000-9,500)\end{array}$ & $\begin{array}{l}\text { Warm dry } \\
\text { lo well. } \\
\text { dralnad }\end{array}$ & $\begin{array}{l}\text { A. laslocarpa } \\
\text { climax or } \\
\text { co.climax wlth } \\
\text { P. angolmannll } \\
\text { (CO) }\end{array}$ & $\begin{array}{l}\text { P. angalmannll } \\
\text { P. manzlos"l } \\
\text { P. contorta } \\
\text { P. llaxllis } \\
\text { P. albicaulls } \\
\text { P. tramuloldes }\end{array}$ & $\begin{array}{l}\text { P. racamosa } \\
\text { P. myrsinilas } \\
\text { S. oraophllus } \\
\text { A. cordllolla } \\
\text { A. angalmannll } \\
\text { L. lanszwerll } \\
\text { P. sacunda }\end{array}$ & $\begin{array}{l}\text { Mauk and } \\
\text { Henderson } 1984 \\
\text { Steela el al. } 1983\end{array}$ \\
\hline $\begin{array}{l}\text { Ables leslocarpel } \\
\text { Polemonlum pulcherrimum H.T. } \\
\text { [A. lastocerpa. } \\
\text { Plcea engelmannill } \\
\text { P. pulcherrimum H.T.] }\end{array}$ & $\begin{array}{l}\text { Mountains ol } \\
\text { south-cantrel } \\
\text { Cotorado } \\
(10,500 \cdot 11,000)\end{array}$ & Cool dry & $\begin{array}{l}\text { A. laslocarpa } \\
\text { co.cllmax with } \\
\text { P. engelmannll }\end{array}$ & P. angelmannll & $\begin{array}{l}\text { P. pulcharrimum } \\
\text { (P. dellcolum) } \\
\text { Vaccinlum spp. } \\
\text { C. leptosapala } \\
\text { Osmorhize oblusa }\end{array}$ & $\begin{array}{l}\text { Komarkova of al. } \\
1988\end{array}$ \\
\hline $\begin{array}{l}\text { Ables leslocarpel } \\
\text { Saxifrage bronchialls H.T. } \\
\text { (Scraa forast) }\end{array}$ & $\begin{array}{l}\text { Mounlains of } \\
\text { northern New } \\
\text { Mexico and } \\
\text { southern Colorado } \\
(10,000 \cdot 11,000)\end{array}$ & Cool dry & $\begin{array}{l}\text { A. laslocarpa } \\
\text { cllmax or } \\
\text { co-cllmax with } \\
\text { P. engelmannll }\end{array}$ & $\begin{array}{l}\text { P. engeimannil } \\
\text { P. menzleshl } \\
\text { P. strobitormis }\end{array}$ & $\begin{array}{l}\text { S. bronchialls } \\
\text { J. communis } \\
\text { R. monilganum } \\
\text { K. crlstata } \\
\text { (K. macrantha) } \\
\text { C. rossll } \\
\text { F. ovalls } \\
\text { (F. virginiana) }\end{array}$ & $\begin{array}{l}\text { Develice ot el. } \\
1986\end{array}$ \\
\hline $\begin{array}{l}\text { Ables leslocarpal } \\
\text { Senocio sangulsorboldes H.T. } \\
\text { S. aengulsorboldes (typic) phaae } \\
\text { Psoudotsuga menziesll phase }\end{array}$ & $\begin{array}{l}\text { Mountalns of } \\
\text { southern New } \\
\text { Mex|co }(\geq 10,000)\end{array}$ & $\begin{array}{l}\text { Cool dry } \\
\text { to well. } \\
\text { drained }\end{array}$ & $\begin{array}{l}\text { A. lasiocarpa } \\
\text { co.climax with } \\
\text { P. engelmannll }\end{array}$ & $\begin{array}{l}\text { P. engelmannil } \\
\text { P. menziesil } \\
\text { P. Iramuloidas }\end{array}$ & $\begin{array}{l}\text { S. sanguisorboides } \\
\text { R. montigenum } \\
\text { Aibes wollli } \\
\text { E. eximius } \\
\text { (E. superbus) }\end{array}$ & $\begin{array}{l}\text { Aloxander at al. } \\
\text { 1984a } \\
\text { Moir end } \\
\text { Ludwig } 1979\end{array}$ \\
\hline
\end{tabular}





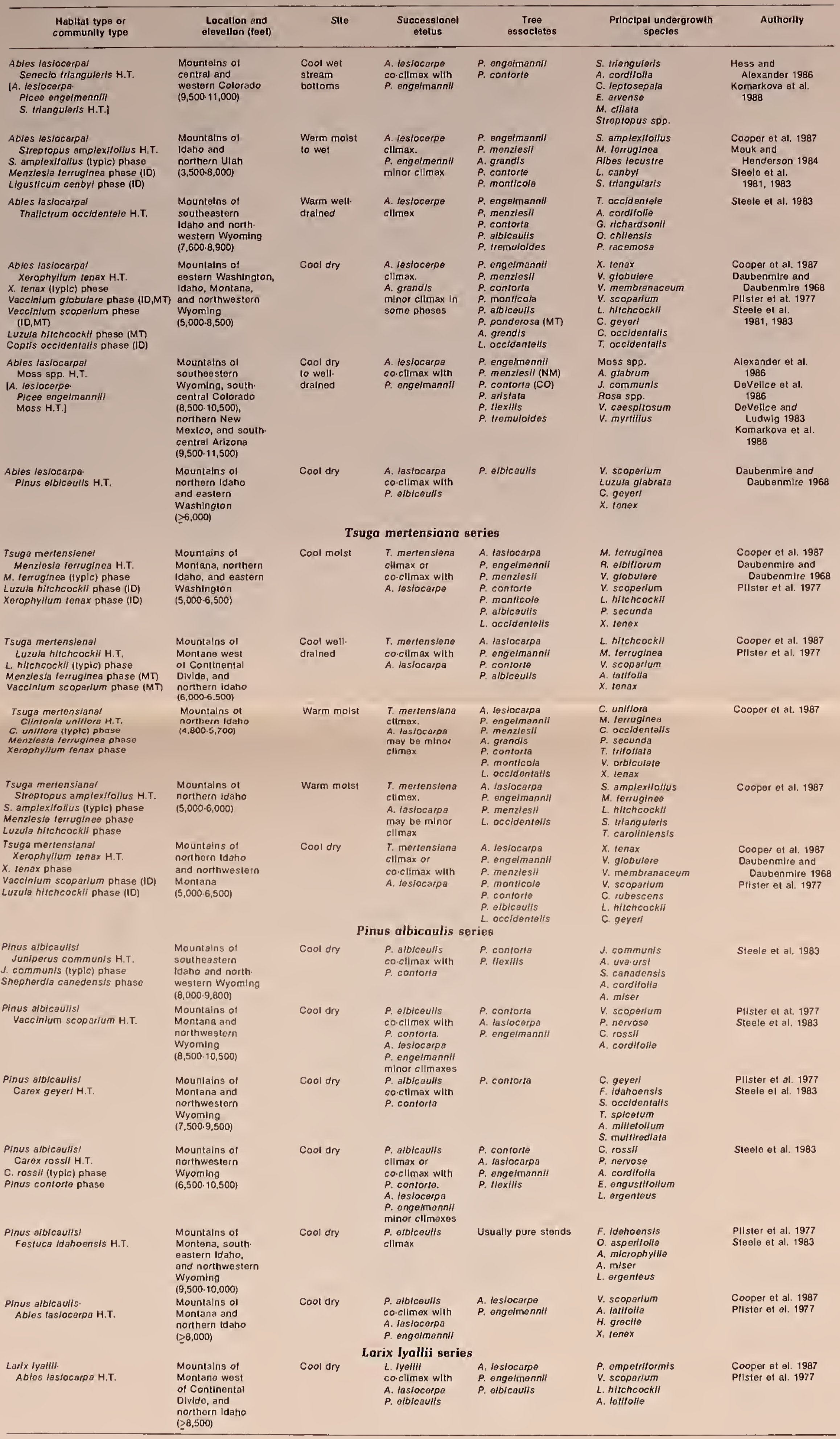





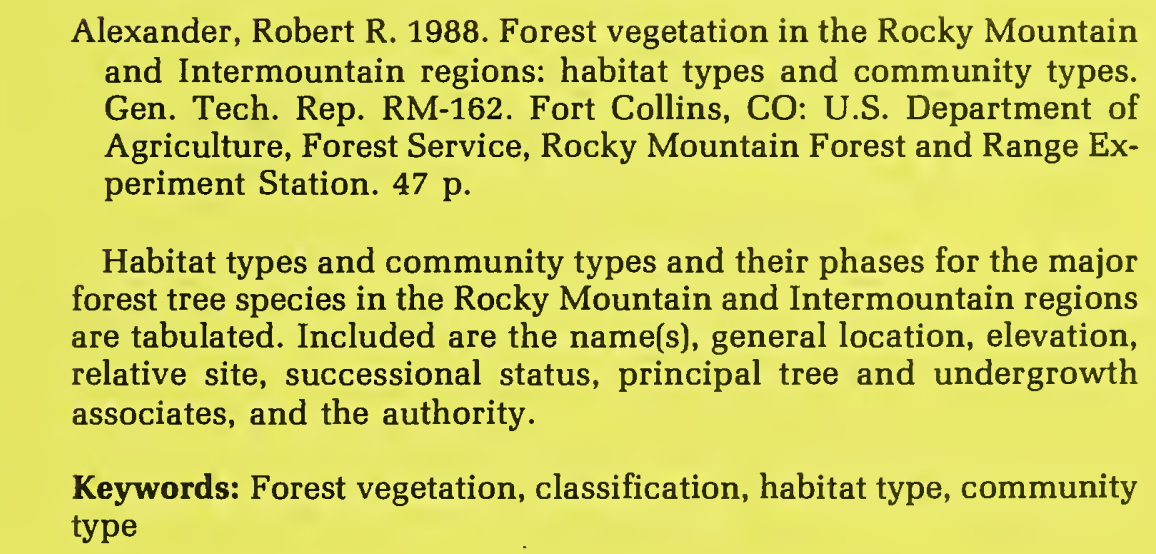

Habitat types and community types and their phases for the major forest tree species in the Rocky Mountain and Intermountain regions are tabulated. Included are the name(s), general location, elevation, relative site, successional status, principal tree and undergrowth associates, and the authority.

Keywords: Forest vegetation, classification, habitat type, community type 


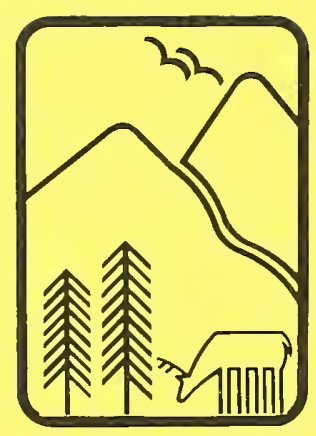

Rocky

Mountains

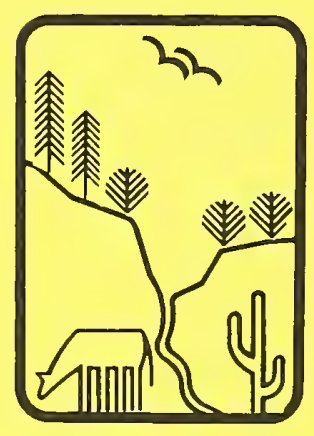

Southwest

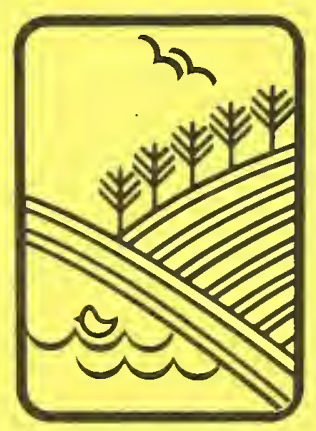

Great Plains
U.S. Department of Agriculture Forest Service

\section{Rocky Mountain Forest and Range Experiment Station}

The Rocky Mountain Station is one of eight regional experiment stations, plus the Forest Products Laboratory and the Washington Office Staff, that make up the Forest Service research organization.

\section{RESEARCH FOCUS}

Research programs at the Rocky Mountain Station are coordinated with area universities and with other institutions. Many studies are conducted on a cooperative basis to accelerate solutions to problems involving range, water, wildlife and fish habitat, human and community development, timber, recreation, protection, and multiresource evaluation.

\section{RESEARCH LOCATIONS}

Research Work Units of the Rocky Mountain Station are operated in cooperation with universities in the following cities:

Albuquerque, New Mexico

Flagstaff, Arizona

Fort Collins, Colorado*

Laramie, Wyoming

Lincoln, Nebraska

Rapid City, South Dakota

Tempe, Arizona

- Station Headquarters: 240 W. Prospect St., Fort Collins, CO 80526 